\title{
Heavy-flavor production in heavy-ion collisions and implications for the properties of hot QCD matter
}

\author{
R. Averbeck \\ ExtreMe Matter Institute EMMI and Research Division, \\ GSI Helmholtzzentrum für Schwerionenforschung, Darmstadt, Germany
}

\begin{abstract}
Hadrons carrying open heavy flavor, i.e. single charm or bottom quarks, are among the key diagnostic tools available today for the hot and dense state of strongly interacting matter which is produced in collisions of heavy atomic nuclei at ultra-relativistic energies. First systematic heavy-flavor measurements in nucleus-nucleus collisions and the reference proton-proton system at Brookhaven National Laboratory's (BNL) Relativistic Heavy Ion Collider (RHIC) have led to tantalizing results. These studies are now continued and extended at RHIC and at CERN's Large Hadron Collider (LHC), where considerably higher collision energies are available. This review focuses on experimental results on open heavy-flavor observables at RHIC and the LHC published until July 2012. Yields of heavy-flavor hadrons and their decay products, their transverse momentum and rapidity distributions, as well as their azimuthal distributions with respect to the reaction plane in heavy-ion collisions are investigated. Various theoretical approaches are confronted with the data and implications for the properties of the hot and dense medium produced in ultra-relativistic heavy-ion collisions are discussed.
\end{abstract}

\section{Contents}

1 Introduction 3

1.1 Strongly interacting matter in ultra-relativistic heavy-ion collisions . . . . . . . 3

1.2 Open heavy flavor in pp and nucleus-nucleus collisions . . . . . . . . . . . 7

2 Open heavy-flavor hadro-production measurements in the pre-RHIC era 11

2.1 Open heavy flavor from fixed target experiments . . . . . . . . . . . . 11

2.2 Open heavy flavor from proton/antiproton colliders . . . . . . . . . 15

3 Open heavy-flavor measurements at RHIC $\quad \mathbf{1 8}$

3.1 Experiments studying heavy-flavor production at RHIC . . . . . . . . . . . 18

3.1.1 The PHENIX approach to open heavy-flavor measurements . . . . . . . . 19

3.1.2 The STAR approach to open heavy-flavor measurements . . . . . . . . . 21

3.2 Reference measurements in pp collisions . . . . . . . . . . . . . . 23

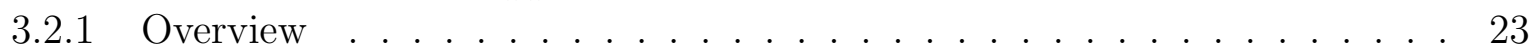

3.2.2 Hadronic heavy-flavor hadron decays . . . . . . . . . . . . . . . . 24

3.2.3 Semielectronic heavy-flavor hadron decays . . . . . . . . . . . . . . 25 
3.2.4 Semimuonic heavy-flavor hadron decays . . . . . . . . . . . . . 32

3.2.5 Total heavy-flavor production cross sections at RHIC . . . . . . . . . . 34

3.3 Heavy flavor in $\mathrm{d}+\mathrm{Au}$ collisions . . . . . . . . . . . . . . . . 34

3.4 Heavy flavor in $\mathrm{Au}+\mathrm{Au}$ collisions . . . . . . . . . . . . . . . . 37

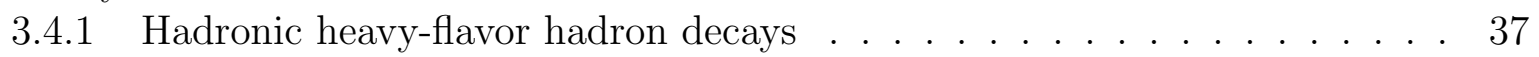

3.4.2 Semielectronic heavy-flavor hadron decays . . . . . . . . . . . . . . . 37

3.4.3 Semimuonic heavy-flavor hadron decays . . . . . . . . . . . . . . 50

4 Open heavy-flavor measurements at the LHC 52

4.1 Experiments studying heavy-flavor production at the LHC . . . . . . . . . . 552

4.1.1 The ALICE approach to open heavy-flavor measurements . . . . . . . . . 53

4.1.2 The ATLAS approach to open heavy-flavor measurements . . . . . . . . 55

4.1.3 The CMS approach to open heavy-flavor measurements . . . . . . . . . 555

4.1.4 The LHCb approach to open heavy-flavor measurements . . . . . . . . . 56

4.2 Reference measurements in pp collisions . . . . . . . . . . . . . . . 56

4.2.1 Hadronic heavy-flavor hadron decays . . . . . . . . . . . . . . 56

4.2.2 Semielectronic heavy-flavor hadron decays . . . . . . . . . . . . . 58

4.2.3 Semimuonic heavy-flavor hadron decays . . . . . . . . . . . . . . . . 61 . . . . . . . . . . . . . . . . . .

4.2.4 FONLL-driven $\sqrt{s}$ scaling . . . . . . . . . . . . . . . 63 . . . . . . . . . .

4.3 Heavy flavor in $\mathrm{Pb}+\mathrm{Pb}$ collisions . . . . . . . . . . . . . . . 63

4.3.1 Hadronic heavy-flavor hadron decays . . . . . . . . . . . . . . 63

4.3.2 Semileptonic heavy-flavor hadron decays . . . . . . . . . . . . . . . . 67

5 Summary and outlook $\quad 69$ 


\section{Introduction}

\subsection{Strongly interacting matter in ultra-relativistic heavy-ion colli- sions}

Quantum chromodynamics (QCD) is the underlying theory of the strong interaction [1]. At sufficiently high temperature, $T$, or baryo-chemical potential, $\mu_{\mathrm{B}}$, QCD inspired model calculations predict a phase transition of strongly interacting matter from a system of hadrons to a deconfined medium, dubbed a quark-gluon plasma (QGP), in which the relevant degrees of freedom are of partonic nature [2 4]. At zero baryo-chemical potential, corresponding to zero net baryon density, QCD calculations on a discretized space-time lattice [5-7] indicate that the phase transition is of the cross over type 8 10]. The critical temperature, $T_{\mathrm{C}}$, is still not known precisely even for $\mu_{\mathrm{B}}=0$. Values in the range $150<T_{\mathrm{C}}<190 \mathrm{MeV}$, corresponding to an energy density in the vicinity of $1 \mathrm{GeV} / \mathrm{fm}^{3}$, have been quoted [11, 12]. However, even larger values in the range $180<T_{\mathrm{C}}<200 \mathrm{MeV}$ can not be excluded [13]. For nonzero baryo-chemical potential lattice QCD calculations become significantly more difficult. Some calculations predict that the phase transition remains of the cross over type for all values of $\mu_{\mathrm{B}}$. Several others indicate that towards higher $\mu_{\mathrm{B}}$ the phase transition will eventually be of first order. Consequently, this would imply the existence of a critical point in the phase diagram of strongly interacting matter. At very high $\mu_{\mathrm{B}}$ the presence of more exotic phases, e.g. color superconducting forms of strongly interacting matter, is expected [14 16]. A schematic QCD phase diagram is shown in Fig. 1.

Strongly interacting matter at extreme temperature and/or density is not only of fundamental interest in the context of mapping out the QCD phase diagram, but it is also of astrophysical relevance. The early universe is believed to have spent its first few microseconds after the big bang in the state of a quark-gluon plasma at high temperature and zero baryo-chemical potential. Hadronization took place when the universe expanded and cooled down below $T_{\mathrm{C}}$. QGP matter at high density and moderate temperature might be present today inside of neutron stars [17], or it might be created briefly during the core collapse of a supernova explosion [18,19].

Collisions of heavy atomic nuclei at ultra-relativistic energies provide the unique opportunity to investigate experimentally the properties and the dynamics of hot and dense QCD matter in the laboratory, because only in such collisions the necessary temperature or density can be reached.

First "circumstantial evidence" for the production of a quark-gluon plasma was reported [20] from the fixed target heavy-ion program at the CERN Super Proton Synchrotron (SPS), where collisions of lead nuclei $(\mathrm{Pb}+\mathrm{Pb})$ were investigated at energies per nucleon-nucleon pair up to $\sqrt{s_{\mathrm{NN}}}=17.3 \mathrm{GeV}$ in the center of mass frame. Experiments with colliding gold nuclei $(\mathrm{Au}+\mathrm{Au})$ at $\sqrt{s_{\mathrm{NN}}}=200 \mathrm{GeV}$ at the dedicated BNL Relativistic Heavy Ion Collider (RHIC) have substantiated these findings and have led to a first quantitative characterization of the properties of the QGP [21 24$]$, revealing some surprises. Here, only some of the most striking observations are briefly summarized.

The initially produced fireball has such a high temperature and density that the partons, i.e. quarks and gluons, equilibrate on a time scale of less than $1 \mathrm{fm} / c$. Large pressure gradients in the system lead to a hydrodynamic evolution of the fireball which subsequently expands, cools down, and undergoes hadronization. When the system is cold and dilute enough, the hadrons freeze out chemically and thermally and stream to the detectors. It is important to note that the geometrical shape of the initial equilibrated partonic fireball is asymmetric for 


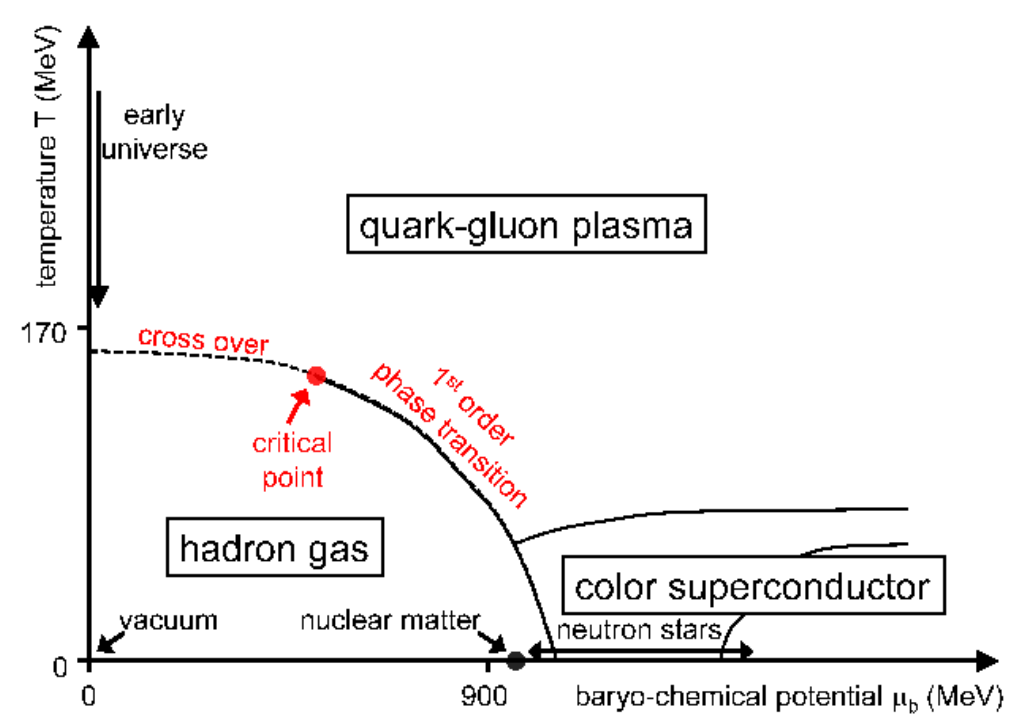

Figure 1: Schematic phase diagram of strongly interacting matter for two massless quarks as a function of the temperature $T$ and the baryo-chemical potential $\mu_{\mathrm{B}}$.

collisions with non-zero impact parameter, exhibiting an almond-like shape averaged over many collisions. Consequently, the pressure gradients driving the expansion are asymmetric as well. Thus the initial spatial anisotropy is translated into an azimuthal anisotropy in momentum space of the produced hadrons. Originally, this anisotropy was quantified based on a Fourier expansion of the momentum distribution

$$
E \frac{d^{3} N}{d^{3} p}=\frac{d^{3} N}{p_{\mathrm{t}} d \phi d p_{\mathrm{t}} d y} \sum_{n=0}^{\infty} 2 v_{\mathrm{n}} \cos \left[n\left(\phi-\Phi_{\mathrm{R}}\right)\right]
$$

where $\phi$ is the azimuthal emission angle of a particle with respect to the orientation of the reaction plane $\Phi_{\mathrm{R}}$ in a given collision [25]. This plane is defined by the beam direction and the impact parameter vector connecting the centers of the colliding nuclei in the transverse plane. While the short axis of the initial almond-like shape of the fireball is aligned with the reaction plane, the long axis is perpendicular to the latter. The harmonic coefficients, $v_{\mathrm{n}}$, quantify the strength of the anisotropy. The second harmonic, $v_{2}$, is also called the elliptic flow, and it is believed to be sensitive to the early dynamics in the collision. Recently, it was recognized that fluctuations of the initial state geometry are important as they can generate higher $n$ th-order flow components [26 32], in particular odd- $n$ components, when measured with respect to the proper initial-state planes of symmetry $\Phi_{n}\left[33\right.$ instead of $\Phi_{R}$. For a given initial state configuration and equation of state of the expanding medium the laws of ideal or viscous relativistic hydrodynamics predict the momentum space azimuthal asymmetry. The observed good agreement between the predictions [40-42] and the measured $v_{2}$ of various hadron species imply that the produced medium expands collectively almost like a perfect fluid, i.e. a fluid with a small shear viscosity to entropy density ratio, $\eta / s$, close to the conjectured [43] quantum lower bound of $\eta / s=1 / 4 \pi$. Precision measurements of $v_{3}$ or even higher orders of the azimuthal anisotropy [34 39] should improve the sensitivity to the viscosity of the medium [30].

This partonic fluid produced in $\mathrm{Au}+\mathrm{Au}$ collisions at $\mathrm{RHIC}$ is so dense that quarks and gluons from initial partonic hard scattering processes ("jets") suffer a large energy loss while they propagate through the hot and dense medium. This "jet quenching" gives rise to a strong 
suppression of hadrons originating mainly from jet fragmentation at high transverse momentum, $p_{\mathrm{t}}$, relative to the expectation from a superposition of independent binary nucleon-nucleon collisions. To quantify this suppression the nuclear modification factor $R_{\mathrm{AA}}$ is introduced as

$$
R_{\mathrm{AA}}=\frac{d N_{\mathrm{AA}} / d p_{\mathrm{t}}}{\left\langle N_{\mathrm{coll}}\right\rangle \times d N_{\mathrm{pp}} / d p_{\mathrm{t}}}=\frac{d N_{\mathrm{AA}} / d p_{\mathrm{t}}}{\left\langle T_{\mathrm{AA}}\right\rangle \times d \sigma_{\mathrm{pp}} / d p_{\mathrm{t}}}
$$

where $d N_{\mathrm{AA}} / d p_{\mathrm{t}}$ is the differential invariant yield in nucleus-nucleus collisions and $d N_{\mathrm{pp}} / d p_{\mathrm{t}}$ $\left(d \sigma_{\mathrm{pp}} / d p_{\mathrm{t}}\right)$ is the corresponding differential invariant yield (cross section) in pp collisions. For a given centrality class the average number of binary collisions is denoted by $\left\langle N_{\text {coll }}\right\rangle$ and $\left\langle T_{\mathrm{AA}}\right\rangle$ is the corresponding nuclear thickness function, which relates $\left\langle N_{\text {coll }}\right\rangle$ with the inelastic nucleonnucleon scattering cross section. $\left\langle N_{\text {coll }}\right\rangle$ and $\left\langle T_{\mathrm{AA}}\right\rangle$ are obtained via Glauber model calculations [44, 45] of the collision geometry taking into account the response of the detectors used for the centrality measurement. As expected for particles not participating in the strong interaction, the nuclear modification factor of photons from initial hard scattering processes, e.g. quark-gluon Compton scattering, is consistent with one [46]. Furthermore, this confirms that the Glauber model based calculation of the collision geometry is under control and that, at least at mid-rapidity at RHIC, initial state modifications of the parton distribution functions in nuclear matter, e.g. shadowing, anti-shadowing, or saturation effects, do not play a significant role. For high $p_{\mathrm{t}}$ hadrons from jet fragmentation the value $R_{\mathrm{AA}} \approx 0.2$ measured at mid-rapidity in $\mathrm{Au}+\mathrm{Au}$ collisions at RHIC [21,24, 47] demonstrates the opaqueness of the produced dense fireball. When confronted with these data, theoretical models indicate that initial energy densities beyond $10 \mathrm{GeV} / \mathrm{fm}^{3}$ are reached in the produced fireball [48, 49], clearly in the QGP regime.

The initial temperature of the fireball can not be measured directly. However, an enhanced yield of quasi-real virtual photons ${ }^{1}$ above known hadronic sources, was measured [50] in central $\mathrm{Au}+\mathrm{Au}$ collisions at $\mathrm{RHIC}$. Interpreting this excess as virtual thermal photon emission from the hot fireball, hydrodynamical models allow to infer the initial temperature $T_{\text {init }}$ and thermalization time $\tau_{0}$ of the fireball. Values of $T_{\text {init }} \sim 300-600 \mathrm{MeV}$ and $\tau_{0} \sim 0.6-0.15 \mathrm{fm} / c$ are consistent with the data [51.

The yield of heavy quarkonia in nucleus-nucleus collisions, i.e. bound states of charm or bottom quarks and their antiquarks, was long thought of as an observable directly related to deconfinement in the fireball [52]. It was predicted that color charge screening in a deconfined QGP would prohibit charm quarks and antiquarks to form a bound state, the $\mathrm{J} / \psi$ meson, similar to the process of Debye screening in an electromagnetic plasma [52]. The resulting $\mathrm{J} / \psi$ suppression was indeed observed for the first time in central nucleus-nucleus collisions at the CERN SPS [53]. However, other mechanisms unrelated to deconfinement, e.g. the absorption of quarkonia in cold nuclear matter or their break-up by interactions with other hadrons (co-movers) produced in the collision, could also describe the SPS data reasonably well [54 56] rendering the interpretation inconclusive. Subsequently, it was realized that in a deconfined medium a new mechanism for quarkonium production might become important if a sufficiently large number of heavy quark antiquark pairs is produced initially. Quarkonia could be formed in this case via statistical hadronization at the phase boundary [57, 58] or, earlier, via coalescence in the QGP [59]. While in nucleus-nucleus collisions at the SPS the number of charm quark anti-quark pairs is much smaller than one, leaving no room for a significant contribution to the $\mathrm{J} / \psi$ yield via regeneration processes, the situation is different at RHIC,

\footnotetext{
${ }^{1}$ low-mass $e^{+} e^{-}$pairs with high $p_{\mathrm{t}}\left(m_{e^{+} e^{-}}<0.3 \mathrm{GeV} / c^{2}, 1<p_{\mathrm{t}}<5 \mathrm{GeV} / c\right)$
} 
where more than 10 charm quark anti-quark pairs are produced in a central $\mathrm{Au}+\mathrm{Au}$ collision and, in particular, at the $\mathrm{LHC}$, where more than 100 such pairs are generated in a central $\mathrm{Pb}+\mathrm{Pb}$ collision. Under such conditions $\mathrm{J} / \psi$ suppression might actually turn into an enhancement via regeneration processes [60]. First evidence in this direction was observed at RHIC in the centrality and, in particular, the rapidity dependence of the nuclear modification factor of $\mathrm{J} / \psi$ mesons, $R_{\mathrm{AA}}^{\mathrm{J} / \psi}\left[61 . R_{\mathrm{AA}}^{\mathrm{J} / \psi}\right.$ reaches its maximum value at mid-rapidity in central $\mathrm{Au}+\mathrm{Au}$ collisions. This is at variance with the expectation from suppression via color screening or from destruction through the interaction with co-moving hadrons, which both should reach their maximum near mid-rapidity where the energy density reaches is the largest. In the regeneration picture, however, this maximum of $R_{\mathrm{AA}}^{\mathrm{J} / \psi}$ at mid-rapidity finds its natural explanation in the fact that in this region of phase space the density of charm quarks and anti-quarks is maximal. Still the situation is not conclusive yet, as other possible scenarios might explain the RHIC data. Most likely, the $\mathrm{J} / \psi$ saga will only be settled by decisive measurements from the LHC, where rather dramatic effects due to charmonium regeneration are expected when the total charm production yield is large enough [62].

At the CERN LHC colliding beams of lead nuclei are investigated at unprecedented high energies $\left(\sqrt{s_{\mathrm{NN}}}=2.76 \mathrm{TeV}\right.$ up to now $)$ since November 2010. The measurement of two-pion Bose-Einstein correlations via Hanbury-Brown Twiss interferometry has shown that the volume of the produced fireball is about twice as large as it was observed in $\mathrm{Au}+\mathrm{Au}$ collisions at RHIC and has a longer life time as well [63]. The pseudorapidity density of charged particles produced at mid-rapidity also increases by a factor close to two going from RHIC to the LHC [64], indicating a substantial increase of the initial energy density reached in $\mathrm{Pb}+\mathrm{Pb}$ collisions at the LHC. Remarkably, for various hadron species the elliptic flow strength $v_{2}$ as a function of $p_{\mathrm{t}}$ is the same within uncertainties at RHIC and at the LHC 65 67], which demonstrates that the produced fireball still resembles the properties of an almost perfect fluid, albeit with a large initial energy density. At the LHC, high energy jets in the $\mathrm{TeV}$ range are copiously produced. Such hard probes for the medium are not available at RHIC and, consequently, the measurement of the nuclear modification factor can be extended to much higher $p_{\mathrm{t}}$ in $\mathrm{Pb}+\mathrm{Pb}$ collisions at the LHC compared to $\mathrm{Au}+\mathrm{Au}$ collisions at $\mathrm{RHIC}$. In central collisions, $R_{\mathrm{AA}}$ reaches its minimum value, corresponding to maximum suppression of hadrons from jet fragmentation, for a hadron $p_{\mathrm{t}}$ of about $6-7 \mathrm{GeV} / c[68,69]$. $R_{\mathrm{AA}}$ increases towards higher values of $p_{\mathrm{t}}$ but stays significantly below one even for hadrons with a $p_{\mathrm{t}}$ of $100 \mathrm{GeV} / c$ [69], highlighting the enormous density of the produced medium. Also in the quarkonium sector intriguing new observations have been made already. The nuclear modification factor of $\mathrm{J} / \psi$ measured at forward rapidity $[70$ is significantly larger in central $\mathrm{Pb}+\mathrm{Pb}$ collisions at the LHC compared to $\mathrm{Au}+\mathrm{Au}$ collisions at $\mathrm{RHIC}$, which might indicate regeneration of charmonia from a deconfined medium. In addition, the suppression of various $\Upsilon$ states, i.e. bottomonia, can be studied in a systematic way at the LHC. After first indications for $\Upsilon$ suppression have been reported at RHIC [71], where the different spin states have not been resolved yet, first data from $\mathrm{Pb}+\mathrm{Pb}$ collisions at the LHC show a suppression of the $\Upsilon(1 \mathrm{~S})$ ground state in central collisions at low $p_{\mathrm{t}}[72]$.

For the coming years the most important scientific challenge for the field is the quantitative characterization of the state of QCD matter produced in ultra-relativistic heavy-ion collisions at RHIC and at the LHC. In this context the measurement of observables related to the production of hadrons carrying open heavy flavor can play a unique role as is argued in Section 1.2, where the basic concepts and related theoretical approaches are briefly introduced. Results, relevant for the current discussion, originating from open heavy-flavor measurements in hadronic 
Table 1: Properties of hadrons carrying open heavy flavor with charm or bottom quantum numbers $\mathrm{C}=+1$ or $\mathrm{B}=+1$, respectively $[73$. Given are the valence quark content, the isospin, spin, and parity $\left(\mathrm{I}\left(\mathrm{J}^{\mathrm{P}}\right)\right)$, the hadron mass, the most important decay modes together with their branching ratios (B.R.), and the decay length $c \tau$. Antihadrons with negative $\mathrm{C}$ or $\mathrm{B}$ quantum numbers are not listed explicitly. They decay into equivalent channels with charge conjugate decay products.

\begin{tabular}{lllllll}
\hline Particle & $\begin{array}{l}\text { Quark } \\
\text { content }\end{array}$ & $\mathrm{I}\left(\mathrm{J}^{\mathrm{P}}\right)$ & Mass $\left(\mathrm{GeV} / c^{2}\right)$ & Decay mode & B.R. (\%) & $c \tau(\mu \mathrm{m})$ \\
\hline $\mathrm{D}^{+}$ & $\mathrm{cd}$ & $\frac{1}{2}\left(0^{-}\right)$ & $1.8696 \pm 0.0002$ & $\mathrm{~K}^{-} \pi^{+} \pi^{+}$ & $9.13 \pm 0.19$ & $312 \pm 2$ \\
& & & & $e^{+}$anything & $16.07 \pm 0.30$ & \\
& & & & $\mu^{+}$anything & $17.6 \pm 3.2$ & \\
$\mathrm{D}^{0}$ & $\mathrm{c} \overline{\mathrm{u}}$ & $\frac{1}{2}\left(0^{-}\right)$ & $1.8648 \pm 0.0001$ & $\mathrm{~K}^{-} \pi^{+}$ & $3.87 \pm 0.05$ & $123 \pm 1$ \\
& & & & $e^{+}$anything & $6.49 \pm 0.11$ & \\
& & & & $\mu^{+}$anything & $6.7 \pm 0.6$ & \\
$\mathrm{D}_{\mathrm{s}}^{+}$ & $\mathrm{c} \overline{\mathrm{s}}$ & $0\left(0^{-}\right)$ & $1.9685 \pm 0.0003$ & $\phi \pi^{+}$ & $4.5 \pm 0.4$ & $150 \pm 2$ \\
& & & & $e^{+}$anything & $6.5 \pm 0.4$ & \\
$\mathrm{D}^{*+}$ & $\mathrm{c} \overline{\mathrm{d}}$ & $\frac{1}{2}\left(0^{-}\right)$ & $2.0102 \pm 0.0001$ & $\mathrm{D}^{0} \pi^{+}$ & $67.7 \pm 0.5$ & $(2.1 \pm 0.5) \times 10^{-6}$ \\
$\Lambda_{\mathrm{c}}$ & $\mathrm{udc}$ & $0\left(\frac{1}{2}^{+}\right)$ & $2.2865 \pm 0.0001$ & $\mathrm{pK} \pi^{-} \pi^{+}$ & $5.0 \pm 1.3$ & $60 \pm 2$ \\
\hline $\mathrm{B}^{+}$ & $\mathrm{ub}$ & $\frac{1}{2}\left(0^{-}\right)$ & $5.2792 \pm 0.0003$ & $\mathrm{~J} / \psi K^{+}$ & $0.1013 \pm 0.0034$ & $492 \pm 2$ \\
& & & & $l^{+} \nu_{l}$ anything & $10.99 \pm 0.28$ & \\
$\mathrm{~B}^{0}$ & $\mathrm{~d} \overline{\mathrm{b}}$ & $\frac{1}{2}\left(0^{-}\right)$ & $5.2795 \pm 0.0003$ & $\mathrm{~J} / \psi K_{\mathrm{S}}^{0}$ & $0.0436 \pm 0.0016$ & $455 \pm 2$ \\
& & & & $l^{+} \nu_{l}$ anything & $10.33 \pm 0.28$ & \\
$\mathrm{~B}_{\mathrm{s}}^{0}$ & $\mathrm{~s} \overline{\mathrm{b}}$ & $0\left(0^{-}\right)$ & $5.3663 \pm 0.0006$ & $\mathrm{~J} / \psi \phi$ & $0.14 \pm 0.05$ & $441 \pm 8$ \\
$\mathrm{~b}$ hadrons & & & & $\mathrm{J} / \psi$ anything & $1.16 \pm 0.10$ & \\
\hline
\end{tabular}

collisions from the pre-RHIC era are briefly summarized in Section 2. The main focus of this article is a review of the results on heavy-flavor yields, transverse momentum and rapidity distributions, as well as azimuthal distributions with respect to the reaction plane in heavy-ion collisions at RHIC (Section 3) and at the LHC (Section 4). Various theoretical approaches are confronted with the data and implications for the properties of the hot and dense QCD matter produced in such collisions are discussed. The article closes with a summary of the current status and an outlook to the future open heavy-flavor program at RHIC, LHC, and elsewhere in Section 5 .

\subsection{Open heavy flavor in pp and nucleus-nucleus collisions}

Open heavy-flavor production is experimentally accessible through the measurement of hadrons carrying charm or bottom quarks or through the measurement of their decay products. The most important properties of the relevant hadrons are summarized in Tab. 1 [73].

In contrast to the light $\mathrm{u}, \mathrm{d}$, and $\mathrm{s}$ quarks, both the charm and the bottom quark are "heavy" with (bare) quark masses significantly exceeding the QCD scale parameter $\Lambda_{Q C D} \simeq 0.2 \mathrm{GeV}$ $\left(m_{\mathrm{c}}=1.29_{-0.11}^{+0.05} \mathrm{GeV}\right.$ and $\left.m_{\mathrm{b}}=4.19_{-0.06}^{+0.18} \mathrm{GeV}[73]\right)$. Thus, the production of a pair of a heavy quark and its antiquark in ultra-relativistic pp collisions proceeds exclusively through 
initial hard partonic scattering processes and, therefore, can be treated theoretically within the framework of perturbative QCD. The unique feature of heavy-flavor production is that such a perturbative treatment is warranted for all momenta since the large quark mass introduces a hard scale even at zero momentum. This is in distinct contrast to gluon and light quark jets which can be treated perturbatively only at high $p_{\mathrm{t}}$.

Consequently, the measurement of heavy-flavor production in pp collisions provides a crucial testing ground for pQCD. In the following, only hadro-production of heavy-flavor hadrons is addressed. A more detailed introduction, which also discusses photoproduction or the production of heavy quarks in $e^{+} e^{-}$collisions, can be found elsewhere 74 76. In this pQCD approach the basic production process of a hadron carrying heavy flavor can be factorized into three components:

- the (non-perturbative) initial conditions. These are mainly determined by the fractional momenta, $x$, the interacting partons carry from the colliding hadrons. The $x$ distributions of various partons inside hadrons have been studied mainly in deep inelastic scattering experiments. They depend on the squared energy-momentum transfer, $Q^{2}$, between the two partons and they have been parametrized in the form of parton distribution functions $(\mathrm{PDF})$.

- the partonic (perturbative) scattering cross section. This can be calculated in perturbative QCD. At leading order (LO), the only processes contributing to heavy-flavor production are gluon fusion and quark anti-quark annihilation. At next-to-leading order (NLO) processes such as gluon splitting or flavor excitation have to be considered in addition. State of the art perturbative calculations of heavy-flavor production go even further in the sense that they calculate cross sections at fixed order with a next-to-leading-log resummation of higher orders in $\alpha_{\mathrm{s}}$.

- the (non-perturbative) fragmentation of the heavy quarks into heavy-flavor hadrons. Here, two cases have to be distinguished:

1. formation of hadrons carrying open heavy flavor: in this case the heavy quark and antiquark individually fragment into hadrons. The energy of a heavy-flavor hadron with respect to the energy of the initially produced heavy quark is given by the fragmentation function, which is measured in $e^{+} e^{-}$reactions and is assumed to be universal, i.e. the fragmentation of a heavy quark does not depend on the mechanism by which this quark was produced.

2. formation of quarkonium states: typically $1-2 \%$ of the produced heavy quark-antiquark pairs form a bound quarkonium state instead of a pair of hadrons with open heavy flavor. While the physics of quarkonia is not discussed further in this article (see references $[77,80]$ for reviews of this topic instead) it is important to mention that the measurement of the total charm and bottom yields would provide a natural reference for the investigation of charmonium and bottomonium yields.

In the context of this review results from two modern NLO pQCD calculation approaches will be confronted with available open heavy-flavor hadro-production data: Fixed Order calculations with Next-to-Leading-Log resummation (FONLL) [81-83 and calculations within the GeneralMass Variable-Flavor-Numbering Scheme (GM-VFNS) [84, 85]. 
In addition to serving as a precision test of QCD, open heavy-flavor measurements in pp collisions provide a baseline for heavy-flavor studies in heavy-ion collisions, where the heavy quarks propagate through and interact with the produced hot and dense QCD matter.

In the absence of nuclear effects, the heavy-flavor yields in nucleus-nucleus collisions would scale with the number of binary collisions as it is the case for all hard probes. Therefore, departures from binary scaling would indicate nuclear modifications of heavy-flavor observables. Two classes of such modifications have to be distinguished from each other, i.e. initial state effects and final state effects.

Initial state effects result from the fact that the distributions of partons embedded in nuclei are different from the parton distribution functions in nucleons. Depending on $x$ and $Q^{2}$, different nuclear modifications of the parton distribution functions are observed. In the antishadowing region at medium $x$ (around $x \sim 0.1$ ), the parton density in nuclei is larger than in nucleons. The shadowing region is characterized by a depletion of the parton density at low $x$ $\left(x<10^{-2}\right)$ in nuclei relative to nucleons. Other effects, such as gluon saturation, may lead to reduced gluon densities at very low $x$ as well. Reviews of nuclear parton distribution functions, on gluon saturation, and on related issues can be found elsewhere [86 88].

Final state effects are in-medium modifications of heavy-flavor observables due to the presence of hot and dense matter in ultra-relativistic heavy-ion collisions. The main focus of the open heavy-flavor programs at RHIC and at the LHC is the investigation of such modifications of open heavy-flavor observables, aiming to shed light on the properties of the hot QCD medium and the nature of parton-medium interactions. In turn, this requires a solid understanding of the initial state effects such that these and the final state effects can be disentangled. A number of final state effect has been predicted and is investigated experimentally.

The total open heavy-flavor yield is sensitive to modifications of the initial parton densities but it is not expected to be changed significantly via final state effects. Because the charm and bottom quark masses are large enough that secondary, thermal heavy-flavor production mechanisms in the early, hot stages of a heavy-ion collision do not contribute significantly to the total heavy-flavor yield at RHIC. It is an interesting question whether thermal production of heavy quark-antiquark pairs can be observed in $\mathrm{Pb}+\mathrm{Pb}$ collisions at the LHC but, most likely, this mechanism will not provide a large contribution to the total heavy-flavor yield even at LHC energies.

A number of final state effects are expected to leave their footprint on the phase-space distributions of heavy-flavor hadrons. In particular, the interaction of partons with the medium [89] is expected to be sensitive to the medium's energy density via the mechanism of parton energy loss (see 90 for a recent review). This energy loss will reflect itself in a softening of the $p_{\mathrm{t}}$ distributions of heavy-flavor hadrons or their decay products with respect to the same spectra in pp collisions. Prior to systematic heavy-flavor measurements at RHIC, it was generally expected that the dominant energy loss mechanism of heavy quarks would be radiative energy loss via medium induced gluon radiation. Models incorporating this energy loss mechanism provide a reasonable description of the high- $p_{\mathrm{t}}$ suppression of light-flavor hadrons at RHIC [91 97]. However, in QCD the radiative energy loss is not the same for all partons. Gluons exhibit a larger color coupling factor than quarks such that the energy loss of quarks is expected to be smaller than that of gluons. Furthermore, with increasing quark mass induced gluon radiation at small angles with respect to the quark momentum vector is reduced owing to the so-called "dead cone effect" 98]. Radiative energy loss should therefore lead to a distinctive mass hierarchy of high- $p_{\mathrm{t}}$ hadron suppression as quantified by the nuclear modification factor $R_{\mathrm{AA}}$. In the $p_{\mathrm{t}}$ range up to about $10 \mathrm{GeV} / c$, where the heavy quark masses are sizable with respect to 
their momenta, the energy loss is expected to decrease when going from light-flavor hadrons, which originate mostly from gluon or light-quark jets, to hadrons carrying charm and hadrons carrying bottom quarks: $R_{\mathrm{AA}}^{\pi}<R_{\mathrm{AA}}^{\mathrm{c}}<R_{\mathrm{AA}}^{\mathrm{b}}$.

It was pointed out, however, that collisional energy loss via elastic scattering should be comparable in magnitude to radiative energy loss over a wide kinematic region [99]. This additional contribution is more important for heavy than for light quarks and it is included in a number of model calculation which are confronted with data later in this review.

Another class of models aiming at the description of heavy quarks in the QCD medium are Langevin-based transport models [100,101] in which a heavy quark is placed into a thermal medium. In such calculations the interaction is given by elastic collisions, in some approaches 101 mediated by the excitation of D- or B-meson like resonant states in the medium. Heavy-quark diffusion and the damping of the initial non-equilibrium dynamics of heavy quarks in the medium are at the heart of these models, which gives indirect access to the viscosity to entropy density ratio of the QCD medium.

In another approach [102,103], heavy-flavor energy loss via repeated sequential formation of heavy-flavor hadrons and their dissociation in the medium is investigated. In this context, the interplay between the formation time of heavy-flavor hadrons and the expansion dynamics of hot QCD matter plays a key role.

A completely different Ansatz [104] makes use of the AdS/CFT (anti de-Sitter-space/conformalfield theory) conjecture, which claims a correspondence between certain strongly coupled string theories and semiclassical gravitational physics. In this framework, heavy-quark energy loss is described in terms of string drag energy loss.

In most of the approaches listed above it is assumed that the chemical composition of heavyflavor hadrons is the same in pp and nucleus-nucleus collisions. However, in the light-quark sector an enhancement of baryons relative to mesons has been observed at intermediate $p_{\mathrm{t}}$ in central heavy-ion collisions at RHIC [105 107]. This becomes relevant as soon as heavyflavor production is studied via the measurement of decay products of heavy-flavor hadrons, in particular electrons or muons from heavy-flavor semileptonic decays. For example, owing to a smaller semileptonic decay branching ratio and a softer decay lepton spectrum of $\Lambda_{\mathrm{c}}$ baryon compared to D-meson decays, the $R_{\mathrm{AA}}$ of electrons from charm hadrons decays would be smaller than one even without energy loss of charm in the medium 108, 109]. Consequently, not only heavy-flavor mesons but also baryons have to be measured in exclusive decay channels in order to allow for unambiguous conclusions concerning in-medium modifications of heavyflavor observables. Also for precision measurements of the total heavy-flavor production yields in $\mathrm{pp}$ and heavy-ion collisions it is mandatory to directly determine the contribution from heavy-flavor baryons. Unfortunately, until today heavy-flavor baryon yields and spectra could not be measured in heavy-ion collisions.

Further insight into the interaction between heavy quarks and the hot QCD medium can be gained from the measurement of the azimuthal anisotropy of heavy-flavor hadron spectra with respect to the orientation of the reaction plane in heavy-ion collisions. In particular, the elliptic flow parameter $v_{2}$ is expected to be sensitive to the degree of thermalization of heavy quarks within the partonic medium. The measurement of a non-zero $v_{2}$ would certainly be indicative of a strong interaction of heavy quarks with the medium. In addition, heavy-flavor elliptic flow data are useful to benchmark models in which heavy-flavor hadrons are formed via the quark coalescence mechanism. Most of the theoretical approaches introduced above in the context of heavy-quark energy loss in the medium also provide predictions for $v_{2}$ such that a simultaneous measurement of $v_{2}$ and $R_{\mathrm{AA}}$ provides a more stringent test of the underlying physics. 

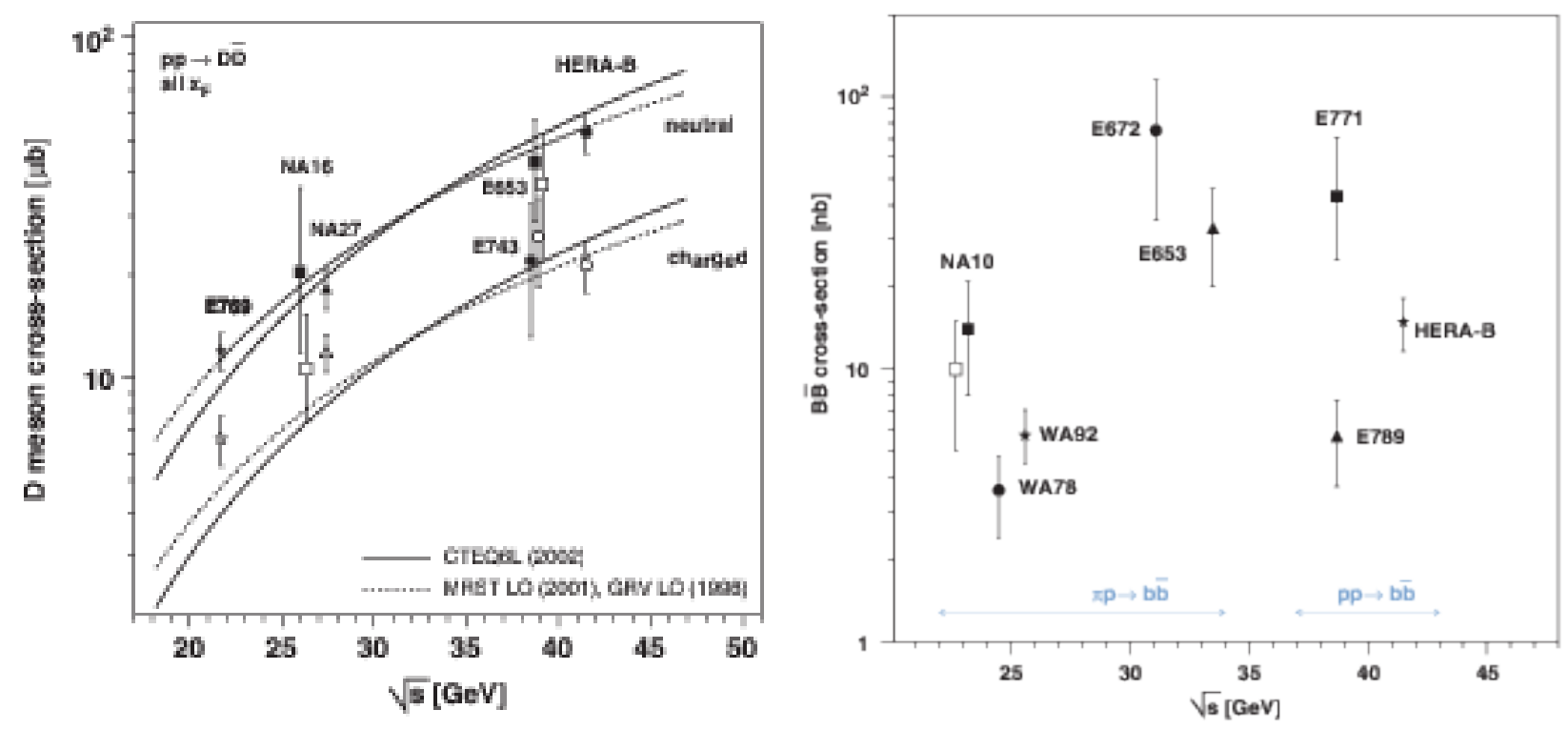

Figure 2: Equivalent pp production cross sections of neutral and charged D-mesons as functions of $\sqrt{s}$ in fixed-target experiments LO pQCD calculation with the PYTHIA event generator using different parton distribution functions are compared with the experimental data (left panel). Corresponding B-meson production cross sections from fixed target $\pi p$ and pp collisions (right panel) (reprinted from Ref. [118).

In summary, the value of heavy-flavor observables in hadronic collisions is twofold. First, in pp collisions perturbative QCD can be tested in a unique way and, thus, a solid experimental and theoretical reference can be prepared which is necessary for corresponding heavy-flavor measurements in nuclear collisions. Second, in collisions of the latter type the interaction of heavy charm and bottom quarks with hot and dense QCD matter is expected to be not only quantitatively but even qualitatively different from the interaction of light quark and gluon jets with this medium. Consequently, heavy-flavor measurements allow a complementary characterization of the parton-medium interactions and of the properties of strongly interacting matter, as it is produced in ultra-relativistic heavy-ion collisions, which can not be achieved otherwise.

\section{Open heavy-flavor hadro-production measurements in the pre-RHIC era}

\subsection{Open heavy flavor from fixed target experiments}

The discovery of hadrons carrying open charm or bottom was preceded in both cases by the discovery of charmonium or bottomonium, respectively. The $\mathrm{J} / \psi$ charmonium state was discovered in the so-called "November revolution" simultaneously in p-Be collisions at the Brookhaven AGS [110] and in $e^{+} e^{-}$collisions at Stanford's storage ring SPEAR [111]. The $\Upsilon$ bottomonium state was discovered in p-nucleus collisions at FNAL [112]. Experimentally, the advantage of the $\mathrm{J} / \psi$ and the $\Upsilon$ compared to open heavy-flavor hadrons is related to the fact that the quantum numbers of these quarkonia coincide with those of the photon. Therefore, they can be produced 
resonantly in $e^{+} e^{-}$colliders for precision measurements of their properties. More important in the context of heavy-ion collisions, the $\mathrm{J} / \psi$ and $\Upsilon$ can decay into dilepton pairs giving rise to the unique experimental signature of a high $p_{\mathrm{t}}$ dilepton pair, which can be triggered on. Both neutral [113] and charged [114] open-charm D mesons were first observed at the SLAC SPEAR storage ring in purely hadronic decays. First evidence for open-bottom production was found with the CLEO [115] and CUSB [116] detectors at the Cornell Electron Storage Ring (CESR) via the measurement of electrons from semileptonic B-meson decays.

In contrast to heavy quarkonia, in particular the $\mathrm{J} / \psi$ meson, which played a crucial rule in the fixed-target heavy-ion program at the CERN SPS, it was only with the advent of high energy nucleus-nucleus colliders, i.e. RHIC and LHC, that open heavy-flavor hadrons could take their role as unique probes for hot QCD matter. For many years open charm and bottom production was in the focus of elementary particle physics only, studied mostly via photo-production or in $e^{+} e^{-}$experiments.

Although single electrons from heavy-flavor hadron decays were first observed in pp collisions at the CERN ISR collider at $\sqrt{s}=52.7 \mathrm{GeV}[117$, actually before the discovery of charm, subsequent studies of open heavy flavor hadro-production were conducted mainly in fixed-target experiments at $\sqrt{s}<50 \mathrm{GeV}$ employing pion and proton beams at the CERN SPS, at FNAL, and at DESY. Most of these measurements, which are reviewed in detail elsewhere [118], were specifically designed for heavy-flavor measurements. As a key feature they included detectors with high spatial resolution in the target region in order to separate the primary collision vertex from secondary heavy-flavor decay vertices. Bubble chambers, emulsions, and silicon tracking telescopes were used for this purpose. Large statistical samples have been collected with pion beams only. The proton beam experiments accumulated much less data, with the $\approx 300$ neutral and charged D-meson events collected by E769 representing the largest statistics data sample [119]. It is interesting to note that not only E769 but also most of the other experiments used nuclear targets, notably $\mathrm{Be}, \mathrm{Al}, \mathrm{Cu}$, and $\mathrm{W}$. To derive equivalent production cross sections for pp (or $\pi \mathrm{p}$ ) reactions a linear dependence of the cross section on the mass number $A$ of the target nucleus was assumed, i.e. $\sigma_{\mathrm{pA}, \pi \mathrm{A}}=A \cdot \sigma_{\mathrm{pp}, \pi \mathrm{p}}$. In Fig. 2 the derived Dand B-meson pair production cross sections in pp or $\pi p$ collisions, extrapolated to $4 \pi$ from the phase space covered in the various experiments, are shown as a function of $\sqrt{s}$. The leadingorder pQCD inspired event generator PYTHIA [120] can be used to describe describe heavyflavor production in the fixed-target energy regime as was demonstrated in Ref. [121]. More detailed and systematic studies have substantiated these findings as summarized in Ref. [118]. Calculations with PYTHIA employing different parton distribution functions are compared with charged and neutral D-meson production cross sections from pp collisions in the left panel of Fig. 2. The fact that higher-order diagrams are absent in PYTHIA is compensated by scaling the calculated cross sections up by an empirical factor, the so-called K-factor. This approach is justified only under the assumption that the kinematical distributions of heavy quarks originating from leading order and higher order processes are similar. Depending on the choice of other model parameters in PYTHIA, K-factors in the range 2.5 - 4.5 are necessary to obtain a satisfactory agreement of the calculation with charm production data. The bottom production cross section is smaller than the charm production cross section by almost three orders of magnitude in this $\sqrt{s}$ range as shown in the right panel of Fig. 2. The experimental uncertainties are large and some of the measurements, e.g. the E771 and E789 results obtained at the same energy, are inconsistent with each other. It is, however, remarkable that PYTHIA calculations, using a K-factor of 2 for bottom production, do not only describe the low energy bottom production cross sections shown in Fig. 2 within a factor of 2 , but they are also in 


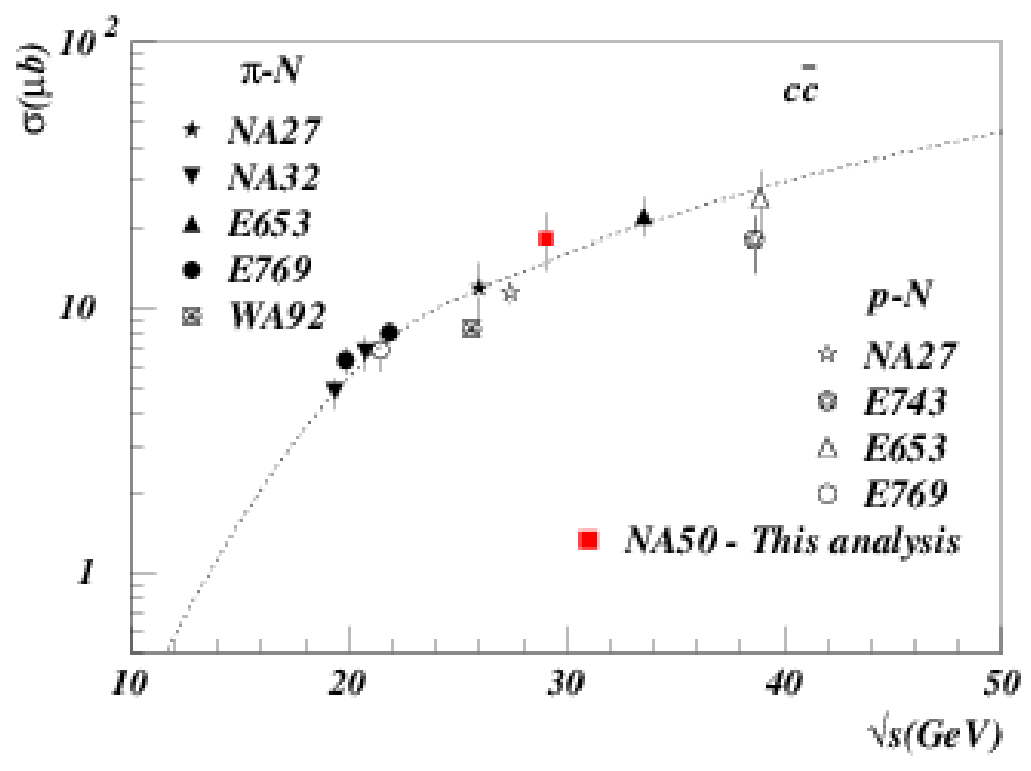

Figure 3: Charm hadro-production cross section in the forward hemisphere $\left(x_{F}>0\right)$ as a function of $\sqrt{s}$ in comparison with the $\sqrt{s}$ dependence of a corresponding PYTHIA calculation. The absolute normalization of the PYTHIA curve was fitted to the experimental data (reprinted from Ref. [124] with kind permission from Springer Science and Business Media).

reasonable agreement with bottom production data measured in $\mathrm{p} \overline{\mathrm{p}}$ collisions at the Tevatron Collider at $\sqrt{s}$ close to $2 \mathrm{TeV}$ as discussed in Ref. [118]. The same systematic analysis of heavy-flavor hadro-production data has demonstrated that for $\sqrt{s}$ values accessible at fixedtarget machines not only the open heavy-flavor absolute production cross sections but also the D-meson kinematical distributions and DD pair correlations can be described reasonably well with PYTHIA.

While in the heavy-ion program at the CERN SPS open heavy-flavor production was never measured directly, the contribution of simultaneous semimuonic decays of correlated D $\bar{D}$ pairs to the dimuon continuum was carefully studied. Particular attention was given to the intermediate mass region (IMR) of the dimuon mass distribution, i.e. the region between the $\phi$ and the $J / \psi$. In addition to open charm decays, the only relevant sources for dimuons in the IMR are the Drell-Yan process and, potentially, thermal dimuon production in the hot QCD matter in nucleus-nucleus collisions. Intermediate mass dimuon production was first measured at the SPS with spectrometers that did not allow for a separation of prompt dimuons from the primary collision vertex and dimuons from displaced, secondary decay vertices characteristic for open charm decays. The NA38 [122] and HELIOS-3 Collaborations [123] measured dimuons in pW, S-U, and S-W collisions at a beam energy of $200 \mathrm{GeV}$ per nucleon. Later, NA38/NA50 investigated p-A collisions, using $\mathrm{Al}, \mathrm{Cu}, \mathrm{Ag}$, and $\mathrm{W}$ as nuclear targets at a beam energy of $450 \mathrm{GeV}$, and $\mathrm{Pb}+\mathrm{Pb}$ collisions at $158 \mathrm{GeV}$ per nucleon [124].

The correlated, opposite-sign IMR dimuon spectra measured by the three experiments in p-A collisions are in good agreement with the sum of the expected Drell-Yan and open charm contributions. In fact, NA50 determined the ratio of dimuons from open charm decays to Drell-Yan dimuons via fits to the mass spectra, and used PYTHIA to infer indirectly the total charm production cross section in pp collisions, assuming that in $\mathrm{p}$-A collisions the total open charm cross section scales with the nuclear target mass number. The resulting charm cross 

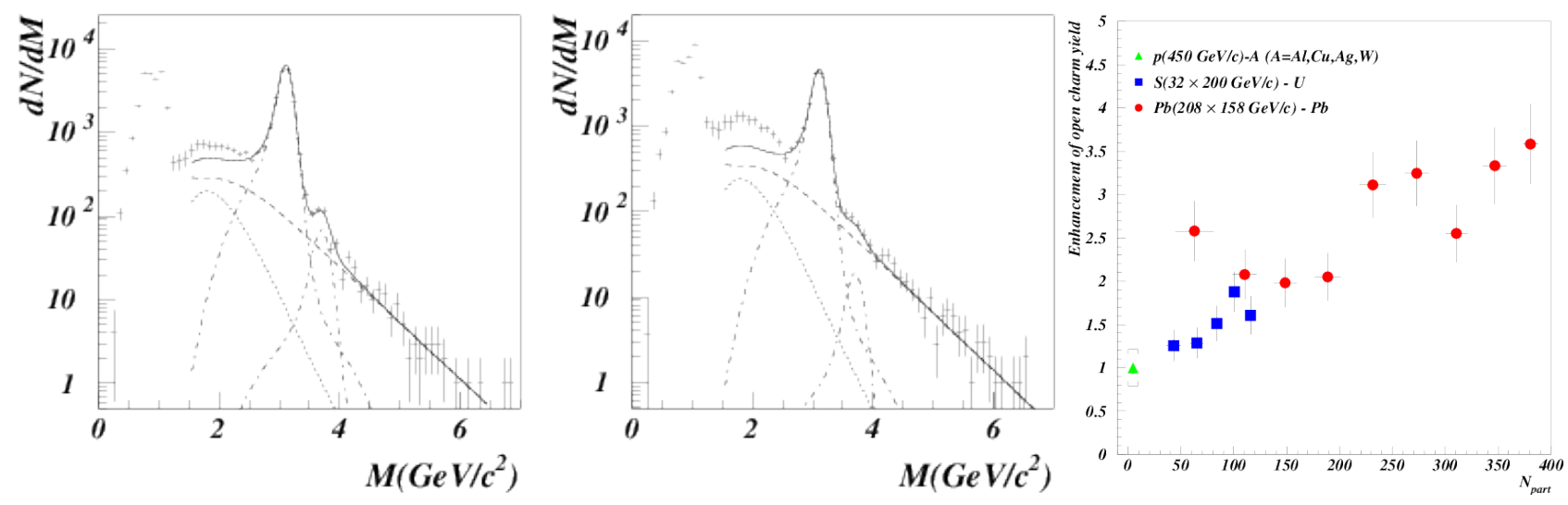

Figure 4: Dimuon yield measured in peripheral $\left(\left\langle N_{\text {part }}\right\rangle=110\right.$, left panel $)$ and central $\left(\left\langle N_{\text {part }}\right\rangle=\right.$ 381, middle panel) $\mathrm{Pb}+\mathrm{Pb}$ collisions at a beam energy of $158 \mathrm{GeV}$ per nucleon in comparison with the contributions from expected sources. The sum (solid) of Drell Yan dimuons (dashed), open charm (dotted), and the dimuon decay of charmonia (dashed-dotted) is not sufficient to saturate the measured IMR dimuon yields. Excess relative to the expected open charm contribution as a function of the number of participants for various collision systems (right panel) (reprinted from Ref. [124] with kind permission from Springer Science and Business Media).

section is shown in Fig. 3 in comparison with direct measurements of the charm production cross section and as a function of $\sqrt{s}$ together with a PYTHIA calculation similar to the one discussed above. The cross section obtained indirectly from the IMR dimuon spectra in p-A collisions agrees reasonably well with the $\sqrt{s}$ dependence obtained from direct measurements.

In contrast, in heavy-ion collisions the measured yields of IMR dimuons exceeded the expectation from Drell-Yan and open charm decay contributions as demonstrated in the two left panels of Fig. 4. The excess, which is clearly visible in the IMR, is more pronounced in central than in peripheral $\mathrm{Pb}+\mathrm{Pb}$ collisions. The centrality dependence is quantified in the right panel of Fig. 4 which shows the excess relative to the expected dimuon yield from open charm decays as a function of the number of participants as measured by NA38 and NA50 in various collision systems.

Among several other potential explanations of the observed IMR dimuon excess two interpretations were discussed as the most likely scenarios, i.e. thermal production as an additional source of IMR dimuons or an increase of the open charm production cross section per nucleon in heavy-ion collisions compared to p-nucleus collisions. The clarification of the origin of the IMR dimuon excess was one of the main goals of the NA60 experiment. The NA60 setup combined the muon spectrometer (MS) previously used by the NA38 and NA50 experiments [125] with a radiation hard silicon vertex tracker (VT) located in a dipole magnet providing a magnetic field of up to $2.5 \mathrm{~T}[126]$. The VT was positioned in between the interaction targets and the upstream hadron absorber in front of the MS. Compared to the previous experiments, the unique feature of this setup was the capability to match tracks of charged particles reconstructed in the VT and the MS both in position AND in momentum space, thus combining the high-rate capability and selective dimuon trigger of the MS with the excellent position and improved momentum resolution provided by the VT. This made it possible to investigate dimuon production in In-In collisions at a beam energy of $158 \mathrm{GeV}$ per nucleon with an unprecedented precision. The 
presence of an IMR dimuon excess consistent with the earlier observations in $\mathrm{Pb}+\mathrm{Pb}$ collisions was confirmed with the NA60 experiment in the In-In collision system [127,128]. Furthermore, it was proven that the excess yield does not originate from a displaced, secondary vertex but is consistent with prompt dimuon emission from the primary collision vertex [127, 128, thus ruling out an enhanced charm production cross section as source responsible for the excess. Instead, the measured properties of the excess dimuons suggest that they are related to thermal radiation from the hot QCD medium.

In summary, open heavy-flavor hadro-production in fixed target experiments was observed to be consistent with expectations from perturbative QCD. This includes data from fixed target heavy-ion collisions, which to date are available only from the SPS. Since open heavy-flavor hadrons were never directly measured in fixed-target heavy-ion collisions it is not precluded that such measurements can provide useful and unique information about the properties of strongly interacting matter in the future. In fact, precision measurements of charm production in nucleus-nucleus collisions close to the production threshold is one of the key goals of the Compressed Baryonic Matter (CBM) experiment [129] at the future FAIR facility.

\subsection{Open heavy flavor from proton/antiproton colliders}

Before the startup of RHIC, open heavy-flavor production was investigated in elementary collisions at other hadron colliders giving access to much higher $\sqrt{s}$ compared to previous fixed target experiments. For the discussion of medium modifications of open heavy-flavor observables in heavy-ion collisions it is useful to investigate whether pQCD calculations provide a theoretical baseline for heavy flavor hadro-production at high collision energies.

The very first open heavy flavor hadro-production measurements at a collider date back to the experimental program at the CERN Intersecting Storage Rings (ISR) which is reviewed in detail elsewhere [130]. Electrons from semileptonic charm hadron decays were observed for the first time in the early 1970s in pp collisions at $\sqrt{s}=52.7 \mathrm{GeV}$ at the ISR 117. However, at the time of these experiments the origin of single electrons measured in the range $1.6<p_{\mathrm{t}}<4.7 \mathrm{GeV} / c$ was not known as the charm quark was not discovered yet. Further measurements of single electrons and lepton pairs (ee and $e \mu$ ) as well as full reconstructions of hadronic decays of open charm hadrons indicated substantial charm production cross sections at ISR energies.

Bottom production at hadron colliders was addressed first in the late 1980s at the CERN Spp $\overline{\mathrm{p}}$. The UA1 Collaboration investigated bottom production at high $p_{\mathrm{t}}\left(p_{\mathrm{t}}>m_{b}\right.$, where $m_{b}$ is the bottom quark mass) in $\bar{p} \bar{p}$ collisions at $\sqrt{s}=546 \mathrm{GeV}$ and $630 \mathrm{GeV}$ [131, 132. For this purpose, events containing single high- $p_{\mathrm{t}}$ muons $\left(10<p_{\mathrm{t}}^{\mu}<40 \mathrm{GeV} / c\right)$ together with a jet or events containing dimuon pairs $\left(2<m_{\mu \mu}<35 \mathrm{GeV} / c^{2}\right)$ were selected. Applying isolation cuts on the single muons and dimuon pairs, respectively, the contributions from bottom hadron decays to the data could be determined statistically. The resulting $p_{\mathrm{t}}$-differential bottom production cross sections from UA1 were compared with next-to-leading-order, i.e. order $\alpha_{s}^{3}$, pQCD calculations which were state of the art calculations at that time [133 136]. In general, a good agreement between data and pQCD calculations was observed within the substantial experimental and theoretical uncertainties. The measurement of open-charm production was difficult at the $\operatorname{Sp} \bar{p} S$. At low $p_{\mathrm{t}}$, no selective triggers were available, and at high $p_{\mathrm{t}}$ it was difficult to separate charm from bottom production. The $\mathrm{D}^{*+}$ meson was the only charmed hadron which could be directly reconstructed with the UA1 experiment in hadronic decays at high $p_{\mathrm{t}}$ in jets [137]. With the UA2 experiment single electrons from semileptonic open charm 

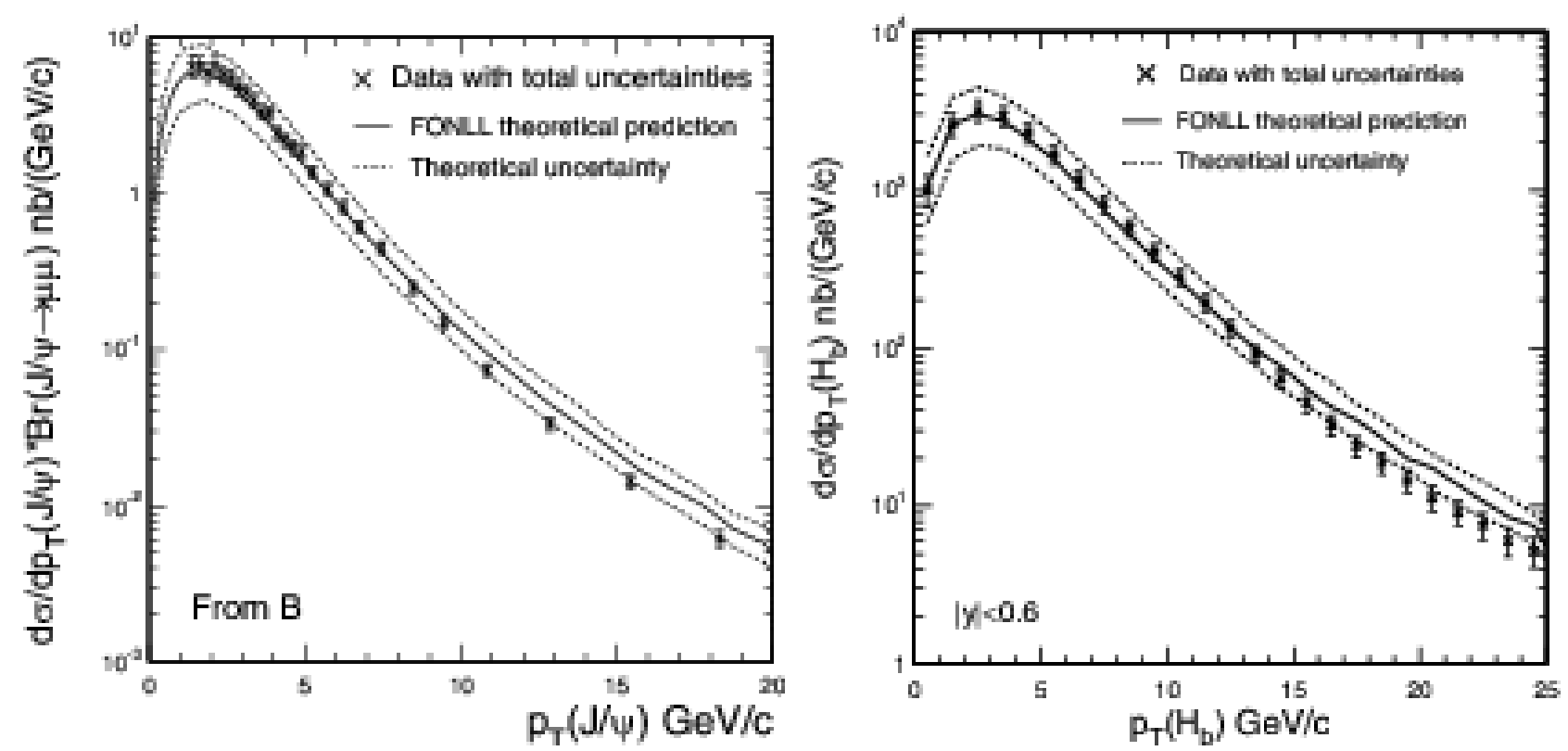

Figure 5: $p_{\mathrm{t}}$-differential cross section of $\mathrm{J} / \psi$ mesons originating from the decay of bottom hadrons integrated over $|y|<0.6$ as measured with the CDF experiment in p $\bar{p}$ collisions at $\sqrt{s}$ $=1.96 \mathrm{TeV}$ (left panel) and the corresponding bottom hadron $p_{\mathrm{t}}$ differential cross section determined from the $\mathrm{J} / \psi$ data (right panel). The plotted $\mathrm{J} / \psi$ cross section includes the branching ratio of $\mathrm{J} / \psi$ mesons in the dimuon decay channel. FONLL pQCD calculations are compared with the data. The solid lines show the central theoretical values and the dashed lines indicate the theoretical uncertainties (reprinted with permission from Ref. [150]; Copyright (2005) by the American Physical Society).

hadron decays were measured in the transverse momentum range $0.5<p_{\mathrm{t}}<2.0 \mathrm{GeV} / c[138$. Only ten such events were observed in this experiment which was the first to use a RICH detector for electron identification at a collider. A detailed review of heavy-quark production

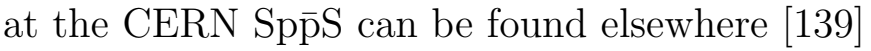

Open heavy-flavor production measurements, mainly focusing again on bottom production, were conducted in the 1990s at the Fermilab Tevatron with the CDF and D0 experiments in $\mathrm{p} \overline{\mathrm{p}}$ collisions first at $\sqrt{s}=1.8 \mathrm{TeV}$ (Run-I) and later at $\sqrt{s}=1.96 \mathrm{TeV}$ (Run-II). The CDF Collaboration reported the first full reconstruction of $\mathrm{B}$ mesons (using the decay $\mathrm{B}^{ \pm} \rightarrow \mathrm{J} / \psi \mathrm{K}^{ \pm}$) at high $p_{\mathrm{t}}$ (above $9 \mathrm{GeV} / c$ ) and at midrapidity $(|y|<1.0$ ) at a hadron collider [140]. Further bottom measurements with the CDF experiment employing electrons [141] and muons [142] from semileptonic bottom decays as well as $\mathrm{B}^{0}$ mesons in the exclusive decay channel $\mathrm{B}^{0} \rightarrow$ $\mathrm{J} / \psi \mathrm{K}^{*}(892)[143$ seemed to have established that bottom production cross sections measured in the $1.8 \mathrm{TeV}$ Run-I at the Tevatron exceeded the expectation from pQCD calculations. While the first bottom-production measurements with the D0 experiment, based on single high- $p_{\mathrm{t}}$ muons at midrapidity, were in reasonable agreement with NLO pQCD calculations [144], later higher statistics measurements of single muons and dimuons [145, 146], and $\mathrm{J} / \psi$ mesons from displaced, secondary vertices at midrapidity [147] followed the trend suggested by the CDF experiment that bottom was produced in excess of the yields expected from NLO pQCD calculations of heavy quark production. This apparent discrepancy between measured bottom production cross sections in $\bar{p} \bar{p}$ collisions and corresponding NLO pQCD calculations posed a long standing 

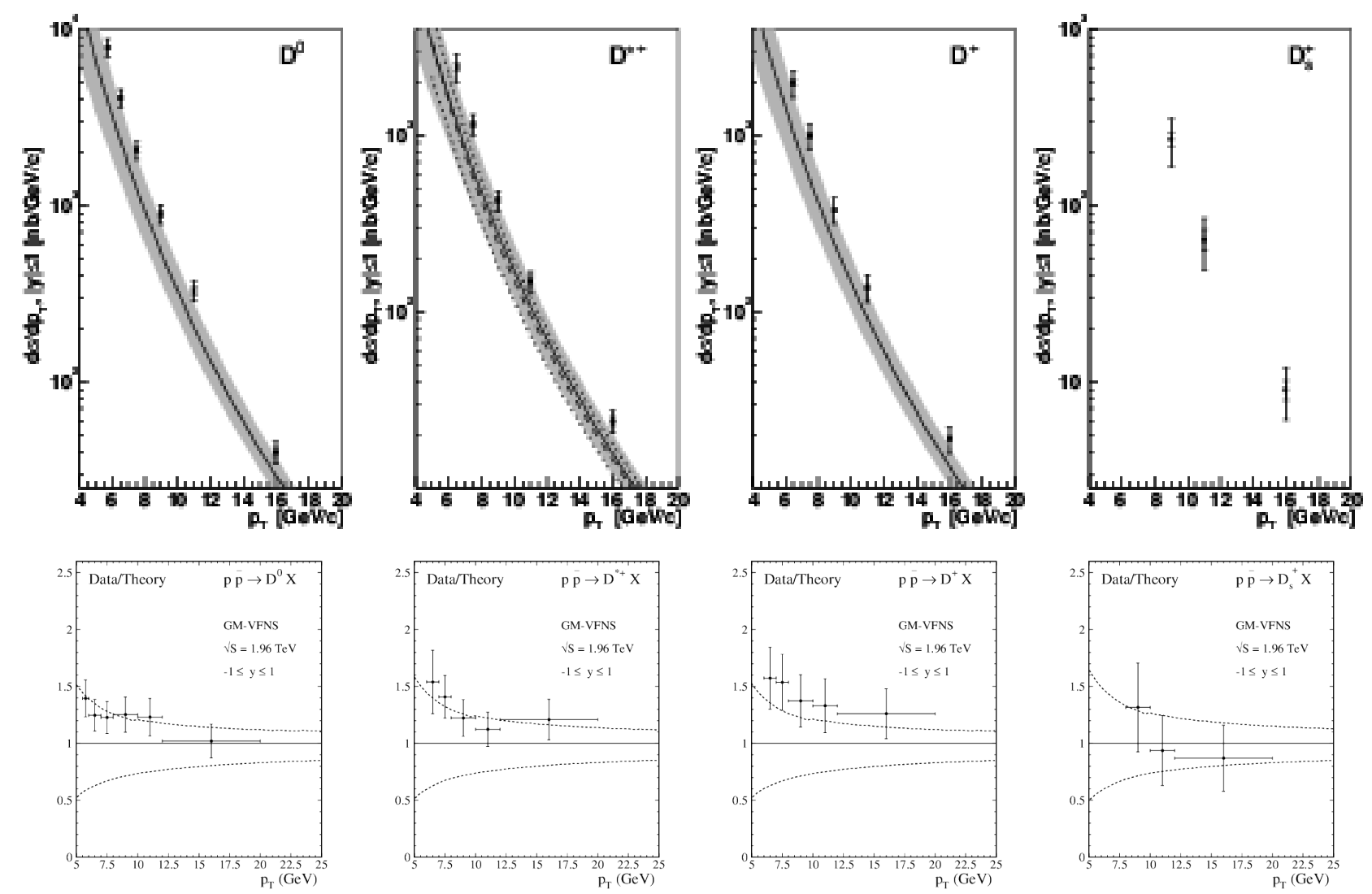

Figure 6: $p_{\mathrm{t}}$-differential cross sections of various D-meson species measured with the CDF experiment in $\mathrm{p} \overline{\mathrm{p}}$ collisions at $\sqrt{s}=1.96 \mathrm{TeV}$ in the rapidity range $|y| \leq 1$. The solid curves are theoretical predictions from FONLL pQCD calculations with the shaded bands indicating the theoretical uncertainties. Predictions for the $\mathrm{D}_{\mathrm{s}}^{+}$cross section are not available from FONLL. Ratios of the measured cross sections and theoretical predictions from GM-VFNS pQCD calculations as functions of $p_{\mathrm{t}}$ (reprinted with permission from Ref. [151] (upper four panels) and Ref. 85] (lower four panels); Copyrights $(2003,2006)$ by the American Physical Society).

puzzle.

As it is documented in detail elsewhere [148, 149] the Tevatron bottom puzzle was finally resolved with a more careful comparison of experimental and theoretical observables and with the availability of much improved data from the Tevatron Run-II at $\sqrt{s}=1.96 \mathrm{TeV}$. In this context, the most important upgrades of the CDF and D0 experiments were related to the trigger systems selecting the events to be recorded for further analysis. Both experiments included silicon vertex detectors in their trigger schemes in Run-II, which allowed them to trigger on the displaced secondary vertices of open heavy-flavor hadron decays. Due to bandwidth limitations the D0 displaced vertex trigger could operate only on preselected events that contained an additional muon. The high-bandwidth displaced vertex trigger of the CDF experiment provided unique access to the measurement of fully hadronic open heavy-flavor hadron decays. Employing this trigger, with the CDF experiment the $p_{\mathrm{t}}$-differential production cross section of $\mathrm{J} / \psi$ from open bottom hadron decays as well as the underlying bottom hadron production cross section was measured down to low $p_{\mathrm{t}}$ in $\mathrm{p} \overline{\mathrm{p}}$ collisions at $\sqrt{s}=1.96 \mathrm{TeV}[150]$. As shown in Fig. 5 this measurement is in excellent agreement with FONLL pQCD calculations as are all 
further open bottom hadron production results from Run-II at the Tevatron.

The CDF silicon vertex trigger did not only allow for a measurement of open bottom production in a unique way at the Tevatron Run-II but it gave also access to open charm production. The $p_{\mathrm{t}}$-differential production cross sections of open charm mesons were measured [151], at high $p_{\mathrm{t}}$ and at midrapidity $(|y| \leq 1)$, in the fully reconstructed hadronic decays $\mathrm{D}^{0} \rightarrow K^{-} \pi^{+}$, $\mathrm{D}^{*+} \rightarrow \mathrm{D}^{0} \pi^{+}, \mathrm{D}^{+} \rightarrow K^{-} \pi^{+} \pi^{+}, \mathrm{D}_{\mathrm{s}}^{+} \rightarrow \phi \pi^{+}$, and their charge conjugates. The measured cross sections are shown in the upper four panels of Fig. 6. Results from FONLL pQCD calculations [81, 82 are plotted in comparison with the data except for the case of the $\mathrm{D}_{\mathrm{s}}^{+}$which is not considered explicitly in FONLL. While the calculations agree with the data within the experimental and theoretical uncertainties, a general trend is observed that the measured cross sections lie at the upper edge of the calculated cross sections. This is further demonstrated in the lower four panels of Fig. 6 which show the ratios of the measured cross sections and corresponding pQCD calculations employing the GM-VFNS approach [85.

In summary, open heavy-flavor hadro-production measurements conducted at pp and $\mathrm{p} \overline{\mathrm{p}}$ colliders before the startup of RHIC can be reasonably well described by state of the art pQCD calculation. Given the significant theoretical uncertainties and the fact that open heavy-flavor measurements have been proven to be difficult, it is crucial to establish an experimental open heavy-flavor production baseline from elementary pp collisions at RHIC, at the LHC, and at future accelerators investigating heavy-flavor hadro-production. Furthermore, such experimental baselines have to be confronted with pQCD calculations before open heavy-flavor hadrons can be used with confidence as probes for the medium produced in ultra-relativistic heavy-ion collisions.

\section{Open heavy-flavor measurements at RHIC}

\subsection{Experiments studying heavy-flavor production at RHIC}

RHIC delivers heavy-ion beams since the year 2000. Two of the four heavy-ion experiments at RHIC, PHENIX and STAR, have conducted systematic open heavy-flavor measurements since then, mainly in pp and $\mathrm{Au}+\mathrm{Au}$ collisions at $\sqrt{s_{\mathrm{NN}}}=200 \mathrm{GeV}$, where substantial data sets have been recorded. In addition, data from $\mathrm{d}+\mathrm{Au}$ collisions, which give access to potential cold nuclear matter effects, and from $\mathrm{Cu}+\mathrm{Cu}$ collisions, which help in the investigation of the system size dependencies of open heavy-flavor observables, were investigated.

The results reviewed in this article originate from published measurements with the PHENIX and STAR experiments in their configurations prior to the years 2008 and 2010, respectively. Since then, both PHENIX and STAR have embarked on ambitious detector upgrade programs. Key components in both upgrade projects are precision vertex spectrometers which will allow the separation of tracks originating from displaced, secondary open heavy-flavor hadron decay vertices from primary tracks pointing back to the collision vertex. These improvements of the experimental setups go hand in hand with an upgrade of the RHIC accelerator facility aiming at an increase of the original $\mathrm{Au}+\mathrm{Au}$ luminosity by a factor of five. The open heavy-flavor physics potential of the detector and accelerator upgrades is discussed in detail elsewhere [152]. In the following, the experimental status quo of the PHENIX and STAR experiments as of the years 2007 and 2009, respectively, is briefly summarized. 

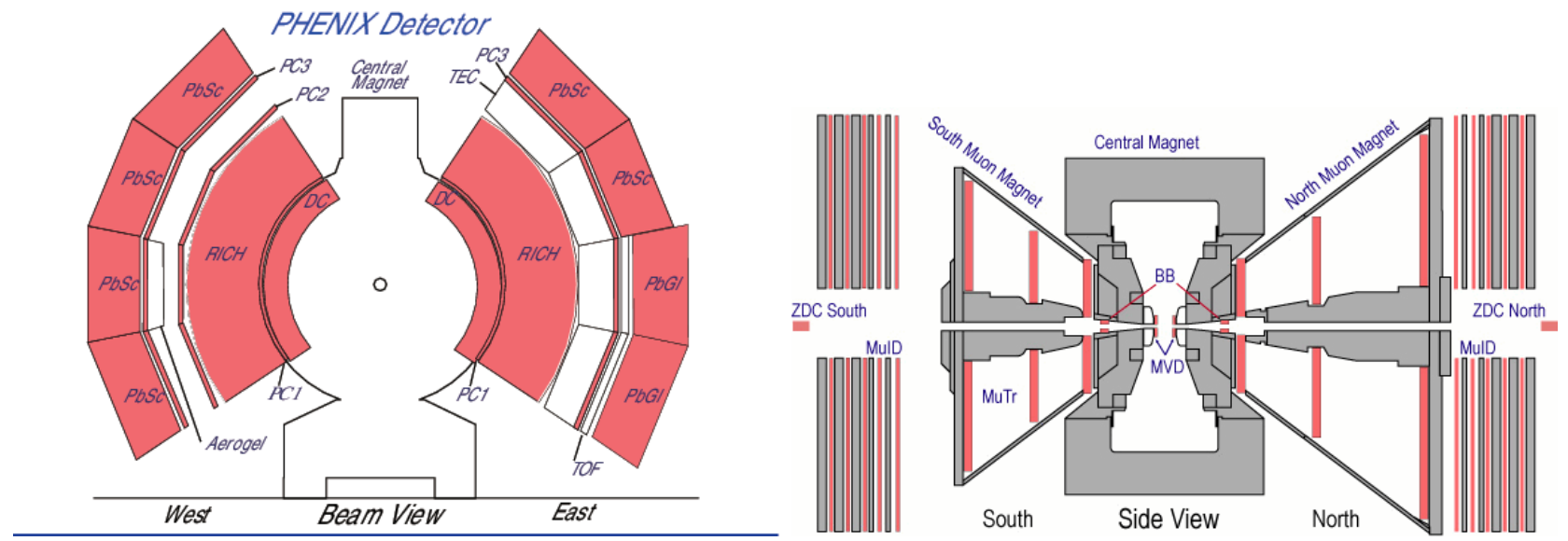

Figure 7: Schematic beam view (left panel) and side view (right panel) of the PHENIX apparatus in the year 2005. Detectors relevant for the heavy-flavor measurements discussed here are the drift chambers (DC) and the pad chambers (PC) for tracking, and the Cherenkov detectors $(\mathrm{RICH})$ and the electromagnetic calorimeters $(\mathrm{PbGl}, \mathrm{PbSc})$ for electron identification in the central arms. The relevant detectors in the forward and backward muon spectrometers are the muon trackers $(\mathrm{MuTr})$ and the muon identifiers (MuID). Global beam-beam counters (BBC) and zero-degree calorimeters (ZDC) are used for event characterization (from Ref. [154]).

\subsubsection{The PHENIX approach to open heavy-flavor measurements}

The PHENIX experiment comprises four spectrometers and a set of global detectors for event characterization. The two central spectrometer arms, each covering $90^{\circ}$ in azimuth, allow the measurement of electrons, photons, and charged hadrons in the pseudorapidity range $|\eta|<0.35$. The momenta of charged particles are measured in the central arms using drift chambers and pad chambers located in a magnetic field which is oriented in parallel to the beam axis around the interaction vertex. For particle identification, time-of-flight detectors, RICH detectors, and electromagnetic calorimeters are employed in the central spectrometers. In the forward and backward regions, two muon spectrometers cover the pseudorapidity ranges $-2.2<\eta<-1.2$ and $1.2<\eta<2.4$ over the full azimuth. Both muon spectrometers comprise a muon tracker with three stations of multi-plane drift chambers placed in a radial magnetic field, and a muon identifier consisting of alternating layers of streamer tubes and steel absorbers. As global detectors, beam-beam counters and zero-degree calorimeters are located at forward and backward rapidity, and they serve a variety of purposes. With these detectors the collision time and vertex position along the beam direction is determined. Furthermore, the first level, minimum bias trigger signals for collisions are derived from the BBC and the ZDC. In addition, they are used to determine the collision centrality and the azimuthal orientation of the reaction place in nucleus-nucleus collisions. Schematic beam and side views of the PHENIX experimental setup are shown in Fig. 7. A detailed description of the PHENIX apparatus can be found elsewhere [153].

In the PHENIX configuration discussed here open heavy-flavor hadrons are not reconstructed exclusively via their hadronic decays. Instead, electrons from heavy-flavor hadron decays are measured with the central spectrometer arms around mid-rapidity in pp and nucleusnucleus collisions. Both the transverse momentum spectra of single electrons and the dielectron mass spectra are inspected for contributions from semileptonic heavy-flavor hadron decays. Cor- 
respondingly, with the forward/backward muon spectrometers single muons from heavy-flavor hadron decays are measured in pp and nucleus-nucleus collisions. Both in the electron and in the muon measurement, the subtraction of background from sources other than heavy-flavor hadron decays poses the most important experimental challenge.

In case of the electron measurements explained in detail in Ref. [155], remaining background from misidentified hadrons does not play a significant role since the combination of Cherenkov detectors and electromagnetic calorimetry guarantees the selection of a rather clean electron candidate sample. From the measured inclusive single electron $p_{\mathrm{t}}$ distributions electron background from non-heavy-flavor sources has to be subtracted. The most important components of this so-called background cocktail, which is calculated using a MonteCarlo hadron decay generator, are Dalitz decays of the $\pi^{0}$ meson and the conversion of photons in detector material, where the majority of photons originates from the $\pi^{0} \rightarrow \gamma \gamma$ decay. It is important to note that, in the PHENIX experiment, special emphasis is put on the minimization of the material budget in the detector aperture, thus minimizing the contribution from photon conversions to the measured electron spectra. With a relevant material budget of less than $1 \%$ of a radiation length, yield ratios of electrons from photon conversion to electrons from light meson Dalitz decays significantly below one are achieved in the PHENIX experiment. Similar Dalitz decay and conversion contributions from other light mesons, notably the $\eta$ meson, are also considered in the electron background cocktail. Furthermore, dielectron decays of light vector mesons ( $\rho$, $\omega, \phi)$ are included although these contributions played a negligible role only. At low $p_{\mathrm{t}}$, a minor contribution from weak kaon decays $\left(K_{\mathrm{e} 3}\right)$ has to be added to the background cocktail. Towards high electron $p_{\mathrm{t}}$ (above $5 \mathrm{GeV} / c$ ) hard quark-gluon Compton scattering processes, contributing to the electron background via virtual photon production and via the conversion of direct real photons in material, play an increasingly important role. In this high $p_{\mathrm{t}}$ region decays of heavy quarkonia $(\mathrm{J} / \psi, \Upsilon)$, and electrons from the Drell-Yan process can not be ignored either, in fact these sources represent about one third of the background electron yield in the range $5<p_{\mathrm{t}}<10 \mathrm{GeV} / c$ in pp and $\mathrm{Au}+\mathrm{Au}$ collisions at $\sqrt{s_{\mathrm{NN}}}=200 \mathrm{GeV}$ at $\mathrm{RHIC}$. In the first electron measurements with PHENIX, which did not reach high electron $p_{\mathrm{t}}$ with good statistics, contributions from $\mathrm{J} / \psi$ and $\Upsilon$ decays as well as from the Drell-Yan process were not included in the electron background cocktail [157 162]. Only with the availability of more precise data these sources were added to the cocktail [155]. The background cocktail from these sources was subtracted from the inclusive electron sample and the difference was identified with electrons from open heavy-flavor hadron decays. A basic requirement for this cocktail subtraction method was the availability of the yields and phase space distributions of all relevant background electron sources, ideally from a measurement in the same apparatus. In addition, the applicability of the cocktail background subtraction method was in general limited to the electron $p_{\mathrm{t}}$ range in which the ratio of signal electrons from heavy-flavor hadron decays to background electrons from other sources was larger than the combined systematic uncertainty of the inclusive electron measurement and the calculated background cocktail. Since the signal-to-background ratio decreases with decreasing electron $p_{\mathrm{t}}$, at low $p_{\mathrm{t}}$ (typically below $2 \mathrm{GeV} / c$ in the PHENIX case) it became advantageous to switch from the cocktail subtraction to the so-called converter subtraction method to determine the yield of electrons from heavyflavor hadron decays. In this complementary approach, additional converter material with a well defined thickness was added to the experimental setup for a fraction of the running time of the experiment. The converter multiplied the yield of "photonic" electrons, i.e. electrons from photon conversions and from Dalitz decays of light mesons, by a known factor, thus providing a measurement of this background contribution which was dominant at low electron $p_{\mathrm{t}}$. A 
detailed description of the measurement of single electrons from heavy-flavor hadron decays with the PHENIX experiment using the cocktail and converter subtraction techniques can be found elsewhere [155].

A disadvantage of single lepton measurements is the fact that contributions from charm and bottom hadron decays can not be separated from each other without additional experimental information. Electron-hadron correlation measurements can provide this missing information because electrons from heavy-flavor hadron decays are always emitted together with hadrons. Since the correlations are not the same in charm and bottom hadron decays they can be used to disentangle the corresponding relative contributions to the electron spectra from heavy-flavor hadron decays [163].

The contributions to the electron-positron mass spectrum from simultaneous semielectronic decays of correlated pairs of hadrons carrying heavy quarks and antiquarks, respectively, was determined with the PHENIX experiment in a cocktail approach as well [164, 165]. Except for photon conversions, the same background sources as listed above for the case of single electrons were considered for the calculation of a background cocktail. After the subtraction of this cocktail background contributions from correlated heavy-flavor hadrons decays became accessible.

Also for the measurement of muons from semileptonic heavy-flavor hadron decays in the forward and backward muon spectrometers of the PHENIX experiment the subtraction of background was crucial [156, 166]. In this case, three background sources were identified as the most important ones. First, decay muons from light hadrons decaying before reaching the first absorber material could not be distinguished experimentally from muons from heavy-flavor hadron decays. Second, punch-through hadrons produced at the collision vertex could penetrate through the MuID layers and, therefore, were included in the inclusive muon candidate sample. Third, muons from the decay of hadrons, which penetrated the muon arm front hadron absorber and decayed in the muon tracker volume, contributed to the inclusive muon candidate sample. The overall background from light hadron sources was quantified via MonteCarlo simulations. The contribution from decay muons could be subtracted statistically. This was possible because the yield of light hadron decay muons exhibited a pronounced vertex position dependence along the beam direction which was not the case for the other sources. Again, a detailed description of the experimental techniques used to isolate the contribution from open heavy-flavor hadron decays to the measured single muon spectra can be found elsewhere 156, 166.

\subsubsection{The STAR approach to open heavy-flavor measurements}

The subdetectors of the STAR experiment relevant in this context were housed inside a solenoid magnet providing a field of $0.5 \mathrm{~T}$. Charged particle tracking relied mostly on a large azimuthally symmetric time projection chamber (TPC) which covered the pseudorapidity range $|\eta|<1.8$. For tracking close to the interaction point and for vertexing the TPC originally was augmented by a silicon vertex tracker (SVT). This device, however, could not be used for a clean separation of decay vertices from open heavy-flavor hadron decays from the primary collision vertex and, hence, it was removed from the STAR setup prior to the RHIC run in the year 2008 to reduce the material budget in front of the TPC inner field cage by a factor of $\approx 10$. For particle identification, the specific energy loss measured in the TPC could be combined with velocity information from a Time-of-Flight barrel which employed multigap resistive plate technology. For electron identification, a barrel electromagnetic calorimeter (EMC) was available in addition. This device included a preshower and a shower maximum detector. As in the PHENIX 


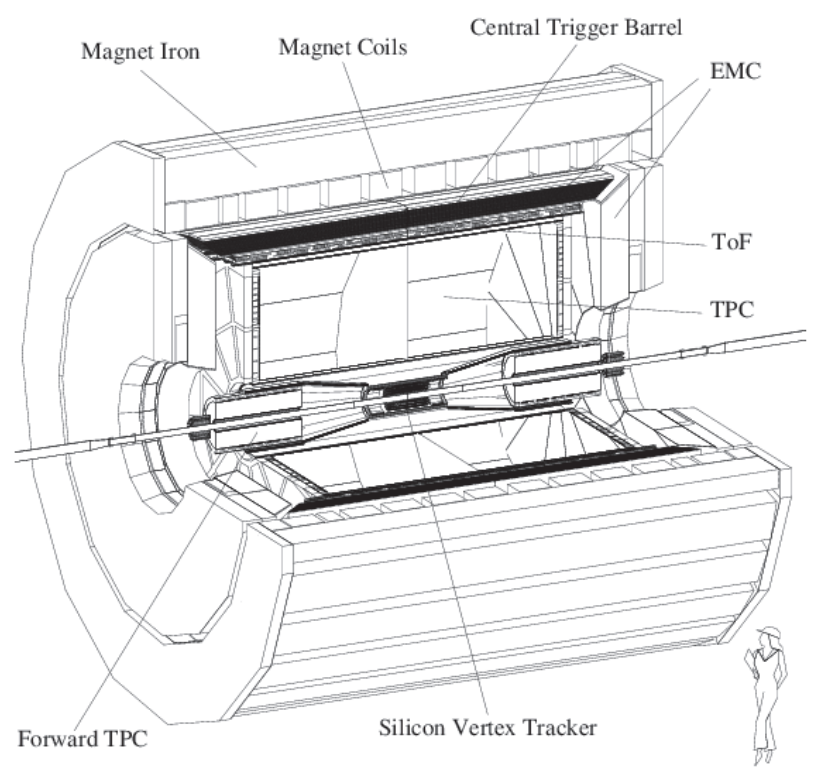

Figure 8: Schematic view of the STAR detector. Charged particles tracks are reconstructed around mid-rapidity in a large volume time projection chamber (TPC), which also contributes to the particle identification via the measurement of the charged particles' specific energy loss $\mathrm{d} E / \mathrm{d} x$. For additional particle identification the TPC is surrounded by a Time-of-Flight barrel (ToF) and an electromagnetic calorimeter (EMC) (from Ref. [168]).

case, beam-beam counters and zero degree calorimeters could be used to provide global event characteristics. A schematic view of the STAR experimental setup is shown in Fig. 8 and a detailed description of the STAR apparatus can be found elsewhere [167].

It is only with the STAR experiment that heavy-flavor hadrons have been fully reconstructed at RHIC via an invariant mass analysis of their hadronic decay products. The main difficulty of such measurements is the substantial combinatorial background from other uncorrelated hadrons. With increasing charged particle multiplicity the resulting signal-to-background ratio becomes worse such that the difficulty of such a measurement increases from pp to proton/deuteron-nucleus to nucleus-nucleus collisions in which signal-to-background ratios of typically 1:1000 had to be coped with in the STAR experiment. With STAR, $\mathrm{D}^{0}$ mesons have been reconstructed in their $\mathrm{K} \pi$ decay channel in $\mathrm{d}+\mathrm{Au}[169]$ and, recently, in pp collisions [170]. A corresponding signal was observed in $\mathrm{Au}+\mathrm{Au}$ collisions at low $p_{\mathrm{t}}$ as well [171]. In addition to the $\mathrm{D}^{0}$ also the $\mathrm{D}^{*+}$ meson has been reconstructed via its $\mathrm{D}^{0} \pi^{+}$decay in pp [172] but not in nuclear collisions. The only possibility to increase the signal-to-background ratio is the clean separation of the secondary decay vertex from the primary collision vertex which has not been achieved with the experimental setup of the STAR experiment discussed here.

Heavy-flavor measurements in $\mathrm{Au}+\mathrm{Au}$ collisions were conducted with the STAR experiment mainly via the semielectronic decays of heavy-flavor hadrons [173], which were also investigated for reference in the pp collision system [173, 174]. Electrons were identified via their specific energy loss $\mathrm{d} E / \mathrm{d} x$ in the STAR TPC, their velocity as measured with the Time-of-Flight detector system, and their response in the electromagnetic calorimeter. A remaining contamination from misidentified hadrons in the electron sample was quantified using the TPC $\mathrm{d} E / \mathrm{d} x$ mea- 
surement, and it was subtracted statistically. As in the PHENIX case, the spectra of electrons from open heavy-flavor decays were obtained via the subtraction of electron background from the measured inclusive electron spectra. Originally, the electron background in the STAR experiment was completely dominated by photon conversions in detector material, in particular in the SVT. With the removal of this detector for the 2008 run at RHIC, the relevant material budget was reduced by a factor $\approx 10$ to just above $1 \%$ of a radiation length, which was close to the material budget in the PHENIX setup. The background from photon conversions and Dalitz decays was, however, determined distinctively different within STAR compared to the PHENIX cocktail and converter subtraction approaches. Electron-positron pairs from the relevant background sources have a very small invariant mass, below the $\pi^{0}$ mass. In contrast to the PHENIX experiment, the large acceptance STAR apparatus provided a reasonably large efficiency $(\approx 60 \%)$ for the reconstruction of these low mass pairs, such that this contribution to the background could be measured and subtracted from the inclusive electron spectrum. Not all of this so-called "photonic background" could be identified with this technique because one of the electrons might have been outside of the detector acceptance or had a momentum to small to be measured. In this case both electrons from the pair were not reconstructed as part of the photonic background. This inefficiency had to be determined precisely through simulations. Contributions from other background sources, e.g. dielectron decays of vector mesons which were not measured directly, were calculated in a cocktail approach. The difference between the measured inclusive electron spectrum and the resulting total background from photonic and other sources was identified with the spectrum of electrons from open heavy-flavor hadron decays.

In pp collisions the azimuthal angular correlations of electrons from open heavy-flavor hadron decays with hadrons or $\mathrm{D}^{0}$ mesons was investigated with the STAR experiment 175]. These procedures allowed a statistical separation of electrons from charm and bottom hadron decays, similar to the approach chosen by PHENIX.

While the STAR experiment does not include a dedicated muon spectrometer muons could be identified at very low $p_{\mathrm{t}}$ (close to $0.2 \mathrm{GeV} / c$ ) via their time of flight and their ionization energy loss in the TPC [171]. Muons from heavy-flavor hadron decays could be separated statistically from the dominant background from weak pion and kaon decays via the measurement of the distance of closest approach of the tracks to the primary collision vertex. The resulting yield of low- $p_{\mathrm{t}}$ muons from heavy-flavor hadron decays provided some sensitivity to the total open charm cross section [171].

\subsection{Reference measurements in pp collisions}

\subsubsection{Overview}

The open heavy-flavor measurements with the PHENIX and STAR experiments in pp collisions at $\sqrt{s}=200 \mathrm{GeV}$ at RHIC aim at two goals. First, these data provide a crucial experimental reference for corresponding measurements in nucleus-nucleus collisions. Such a reference is a key ingredient for the investigation and interpretation of effects due to the presence of a hot and dense medium produced in high-energy nucleus-nucleus collisions. Second, these data provide an important testing ground for perturbative QCD calculations which, due to the large masses of the charm and bottom quarks, should be able to predict open heavy-flavor observables even at low $p_{\mathrm{t}}$.

As will be demonstrated in this section, predictions from NLO pQCD calculations within 

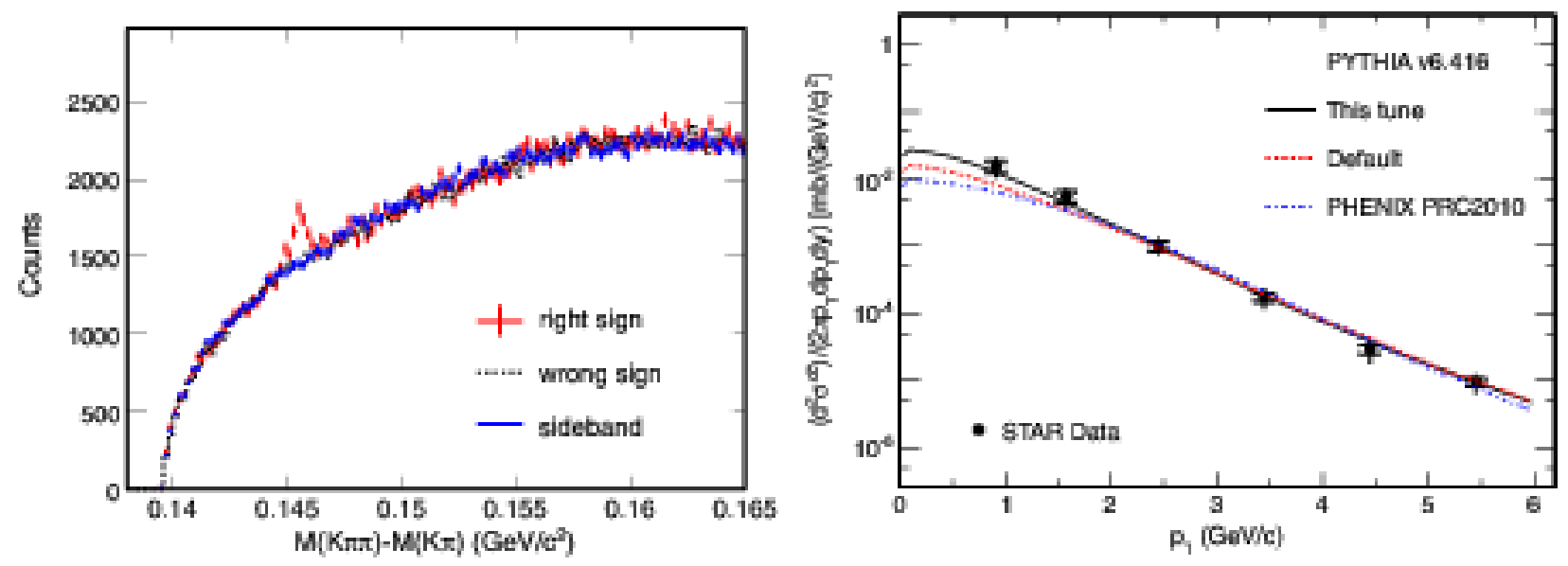

Figure 9: Raw $\mathrm{D}^{*+}$ candidate signal from minimum bias pp collisions at $\sqrt{s}=200 \mathrm{GeV}$ as measured with the STAR experiment. (left panel) Results from two methods to quantify the combinatorial background are shown in addition (see text for details). $c \bar{c}$ production cross section as deduced from the measured $\mathrm{D}^{0}$ and $\mathrm{D}^{*+}$ cross section (right panel). A power-law fit and the $p_{\mathrm{t}}$ differential charm cross section from a FONLL pQCD calculation are shown for comparison (reprinted with permission from Ref. [170]; Copyright (2012) by the American Physical Society).

the FONLL approach [81,82] are in reasonable agreement with essentially all observables related to the production of open heavy-flavor hadrons in pp collisions at RHIC.

\subsubsection{Hadronic heavy-flavor hadron decays}

The only full reconstruction of hadronic decays of heavy-flavor hadrons in pp collisions at $200 \mathrm{GeV}$ at RHIC was the measurement of the $\mathrm{D}^{0} \rightarrow \mathrm{K}^{-} \pi^{+}$decay in the range $0.6<p_{\mathrm{t}}<$ $2.0 \mathrm{GeV} / c$ and the $\mathrm{D}^{*+} \rightarrow \mathrm{D}^{0} \pi^{+}$decay in the range $2.0<p_{\mathrm{t}}<6.0 \mathrm{GeV} / c$ with the STAR experiment [170]. As an example, the left panel of Fig. 9 indicates the quality of the $\mathrm{D}^{*+}$ reconstruction. For minimum bias events, the distribution of the invariant mass difference $\Delta \mathrm{M}=\mathrm{M}(\mathrm{K} \pi \pi)-\mathrm{M}(\mathrm{K} \pi)$ is shown for "right sign" combinations $\left(\mathrm{K}^{\mp} \pi^{ \pm} \pi^{ \pm}\right)$, which contain the $\mathrm{D}^{*+}$ signal, for "wrong sign" combinations $\left(\mathrm{K}^{ \pm} \pi^{\mp} \pi^{ \pm}\right)$, which are combinatorial background, and for $\mathrm{D}^{0}$ "side band" combinations, which select $\mathrm{K} \pi$ pairs that are not consistent with the $\mathrm{D}^{0}$ meson mass. Both the "wrong sign" and the "side band" method reproduce the combinatorial background well. After corrections the measured $p_{\mathrm{t}}$ differential production cross sections for $\mathrm{D}^{0}$ and $\mathrm{D}^{*+}$ mesons in pp collisions at $\sqrt{s}=200 \mathrm{GeV}$ were divided by the charm quark fragmentation ratios $0.565 \pm 0.032$ for $c \rightarrow \mathrm{D}^{0}$ and $0.224 \pm 0.028$ for $c \rightarrow \mathrm{D}^{*+}$, respectively, to convert the meson cross sections to the $c \bar{c}$ cross section. The resulting $p_{\mathrm{t}}$ differential charm cross section is shown in the right panel of Fig. 9 in comparison with the upper and lower limits of the corresponding cross section as predicted with a FONLL pQCD calculation [81, 82, The upper limit of the calculation is consistent with the measured charm hadron cross sections over the full $p_{\mathrm{t}}$ range. The same was observed at higher energy with the CDF experiment at the Tevatron (see Fig. 6) and with the ALICE experiment at the LHC (see Fig. 29).

The $p_{\mathrm{t}}$ differential cross section was parametrized with a power-law function (indicated in the right panel of Fig. 9p and extrapolated to zero $p_{\mathrm{t}}$. Furthermore, the rapidity density of 
the cross section at midrapidity was extrapolated to the full phase space based on calculations taking into account the rapidity dependence of charm production, resulting in an extrapolation factor of $4.7 \pm 0.7$. The rapidity density of the cross section and the total $c \bar{c}$ production cross sections were

$$
\begin{aligned}
\left.\frac{d \sigma_{c \bar{c}}}{d y}\right|_{y=0} & =170 \pm 45(\text { stat. })_{-59}^{+38}(\text { sys. }) \mu \mathrm{b} \\
\sigma_{c \bar{c}} & =797 \pm 210(\text { stat. })_{-295}^{+208}(\text { sys. }) \mu \mathrm{b}
\end{aligned}
$$

\subsubsection{Semielectronic heavy-flavor hadron decays}

With both the PHENIX and STAR experiments extensive measurements of electrons from open heavy-flavor hadron decays have been conducted at RHIC. The layout of the PHENIX apparatus was optimized such that the material budget in the detector aperture was kept at a minimum. The measured ratio [155] of electrons of "non-photonic" origin, i.e. mainly electrons from open heavy-flavor hadron decays, to electrons of "photonic" origin, i.e. electrons from photon conversions in material in the detector aperture and from light meson Dalitz decays, is shown in the left panel of Fig. 10 for pp collisions at $\sqrt{s}=200 \mathrm{GeV}$. Two complementary techniques to separate non-photonic from photonic electrons, i.e. the cocktail subtraction method and the converter subtraction method as described in more detail above, gave consistent results for the non-photonic/photonic electron yield ratio. For $p_{\mathrm{t}}$ above $\approx 2 \mathrm{GeV} / c$ this ratio exceeded one. The original layout of the STAR apparatus included the rather thick (in terms of radiation lengths) SVT detector (see above) which was removed from the experimental setup prior to the 2008 run of RHIC. As demonstrated in the right panel of Fig. 10 the non-photonic/photonic electron yield ratio measured in pp collisions at $\sqrt{s}=200 \mathrm{GeV}$ with the STAR experiment was similar in magnitude to the ratio measured with PHENIX when the SVT was not part of the setup [174]. With the SVT in place, this ratio was substantially smaller, rendering the measurement of electrons from open heavy-flavor hadron decays very challenging, in particular at low $p_{\mathrm{t}}$.

After the first measurements of electrons from open heavy-flavor hadron decays in pp collisions with the PHENIX and STAR experiments at RHIC, over the following few years the precision of the data was improved significantly and the transverse momentum range covered was extended both to lower and higher $p_{\mathrm{t}}$. The current status of these measurements is documented in Fig. 11, which shows the invariant $p_{\mathrm{t}}$ differential cross sections of electrons from heavy-flavor decays measured with the PHENIX experiment (left panel) [155] in the range $0.3<p_{\mathrm{t}}<9.0 \mathrm{GeV} / c$ and with the STAR experiment (right panel) [174]. For the STAR experiment two data sets are shown. In the 2005 RHIC run, with the SVT in place, electrons were measured in the range $1.2<p_{\mathrm{t}}<10 \mathrm{GeV} / c$ (triangle symbols). With additional data from the 2008 run, in which the SVT was removed from the STAR setup, the precision of the measurement could be significantly improved in the range $3<p_{\mathrm{t}}<10 \mathrm{GeV} / c$ (circle symbols). Corresponding predictions of the electron cross section from heavy-flavor decays from a FONLL pQCD calculation [81, 82] are compared with the data in the upper panels of Fig. 11. The ratios of the data and the FONLL calculation are shown in the lower panels of Fig. 11 on a linear scale to allow a better quantitative comparison. The PHENIX and STAR measurements are consistent with each other and, within the experimental and the theoretical uncertainties, which are dominated by the uncertainties of the chosen normalization and factorization scales, the pQCD calculation is in agreement with the data. However, a clear tendency is observed 

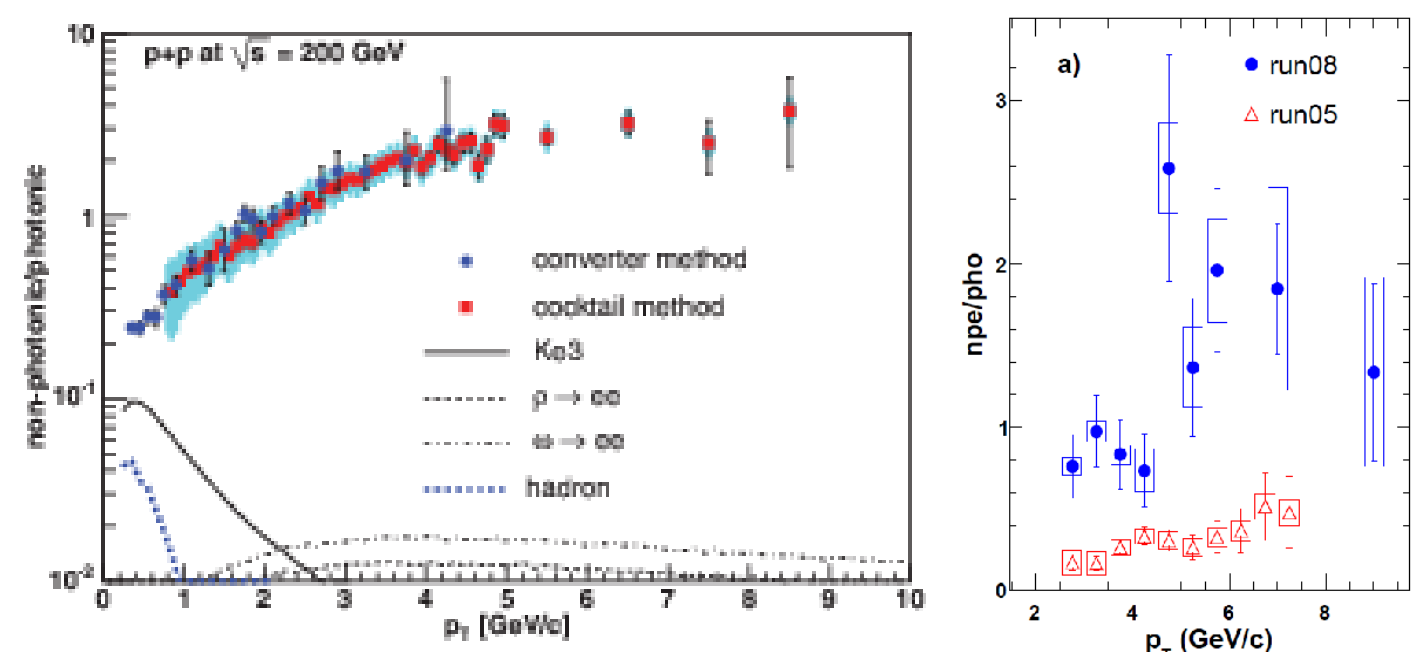

Figure 10: Ratios of electrons from "non-photonic" sources to electrons from "photonic" sources measured in pp collisions at $\sqrt{s}=200 \mathrm{GeV}$ with the PHENIX experiment (left panel) and the STAR experiment (right panel) at RHIC. The "non-photonic" electron spectrum is dominated by electrons from open heavy-flavor hadron decays. Contributions from other sources do not play an important role as demonstrated for the PHENIX case in the left panel (taken from Ref. [155]). The important impact of the material budget is shown in the right panel for the STAR experiment, in which the material budget was reduced significantly in the 2008 RHIC run compared to the 2005 run (see text for details) (reprinted with permission from Ref. [174]; Copyright (2011) by the American Physical Society).

that the measured cross sections are close to the upper limit of the predicted cross section, consistent with the STAR D-meson measurement (see Fig. 9).

According to the FONLL calculation the electron spectrum from heavy-flavor hadron decays is completely dominated by charm decays at low $p_{\mathrm{t}}$, which is expected because of the much smaller total bottom production cross section compared to charm and, in addition, because of the harder bottom decay electron spectrum compared to electrons from charm decays. Therefore, from the measured $p_{\mathrm{t}}$ differential cross section of electrons from heavy-flavor hadron decays the total charm production cross section could be determined. The necessary extrapolation of the measured electron cross section from the $p_{\mathrm{t}}$ and rapidity intervals covered with the PHENIX experiment to the full phase space was done in three steps. First, the measured invariant cross section was extrapolated to zero $p_{\mathrm{t}}$ to obtain the integrated electron cross section per unit rapidity at midrapidity over all $p_{\mathrm{t}}$. The $p_{\mathrm{t}}$ range covered by the PHENIX data included more than $50 \%$ of the total electron yield from charm decays at midrapidity. Second, the integrated electron cross section was converted into a charm cross section, taking into account the effective charm to electron branching ratio and a kinematic correction factor to account for the different rapidity distributions of $\mathrm{D}$ mesons and their decay electrons. Third, the resulting rapidity density of the cross section at midrapidity was extrapolated from midrapidity to the full phase space. A systematic uncertainty of $15 \%$ was quoted for the extrapolation factor which was $\approx 6.6$. Details concerning this procedure can be found elsewhere [155]. The resulting rapidity density of the charm production cross section at midrapidity and the total charm production cross section from the electron measurement with PHENIX are 

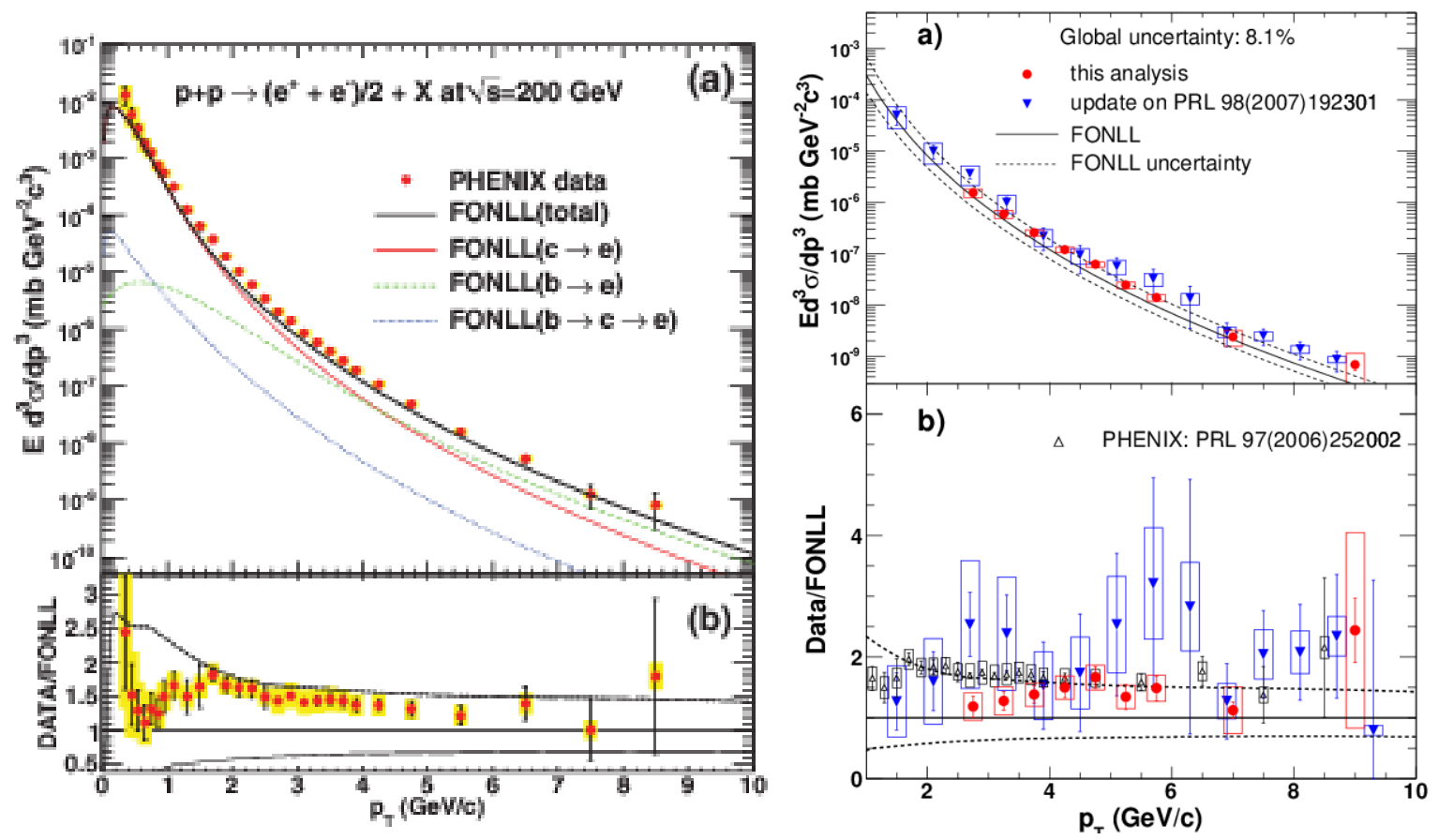

Figure 11: Invariant $p_{\mathrm{t}}$ differential production cross section of electrons from open heavy-flavor hadron decays measured in pp collisions at $\sqrt{s}=200 \mathrm{GeV}$ with the PHENIX experiment (left panels) and the STAR experiment (right panels) at RHIC. The upper panels in both plots include a comparison of the data with corresponding cross sections from a FONLL pQCD calculation. The lower panels show the ratio of the data and the calculation, where the upper (lower) curves indicate the upper (lower) limit of the FONLL calculation. More details are provided in the text (reprinted with permission from Refs. [155] (left) and [174] (right); Copyright (2011) by the American Physical Society).

$$
\begin{aligned}
\left.\frac{d \sigma_{c \bar{c}}}{d y}\right|_{y=0} & =119 \pm 12(\text { stat. }) \pm 38(\text { sys. }) \mu \mathrm{b} \\
\sigma_{c \bar{c}} & =551 \pm 57(\text { stat. }) \pm 195(\text { sys. }) \mu \mathrm{b} .
\end{aligned}
$$

Unfortunately, the electron data measured with the STAR experiment did not extend to low enough electron $p_{\mathrm{t}}$, such that an extrapolation to the full phase space suffered from large systematic uncertainties and a sensible total charm production cross section from the electron measurement alone could not be quoted.

With increasing electron $p_{\mathrm{t}}$ the contribution from bottom decays grows. According to the FONLL calculation, at about $4 \mathrm{GeV} / c$ the contributions from charm and bottom decays are similar, and towards higher $p_{\mathrm{t}}$ bottom decays become the dominant source of electrons from heavy-flavor hadron decays.

It is important to disentangle the charm and bottom decay contributions experimentally such that the FONLL pQCD predictions can be tested separately, and to provide individual references for the decay electron spectra from the two heavy quark species in $\mathrm{Au}+\mathrm{Au}$ collisions at RHIC. Both in PHENIX and STAR such separation techniques were developed based on electron-hadron correlation measurements. Due to the fact that the combinatorial background 

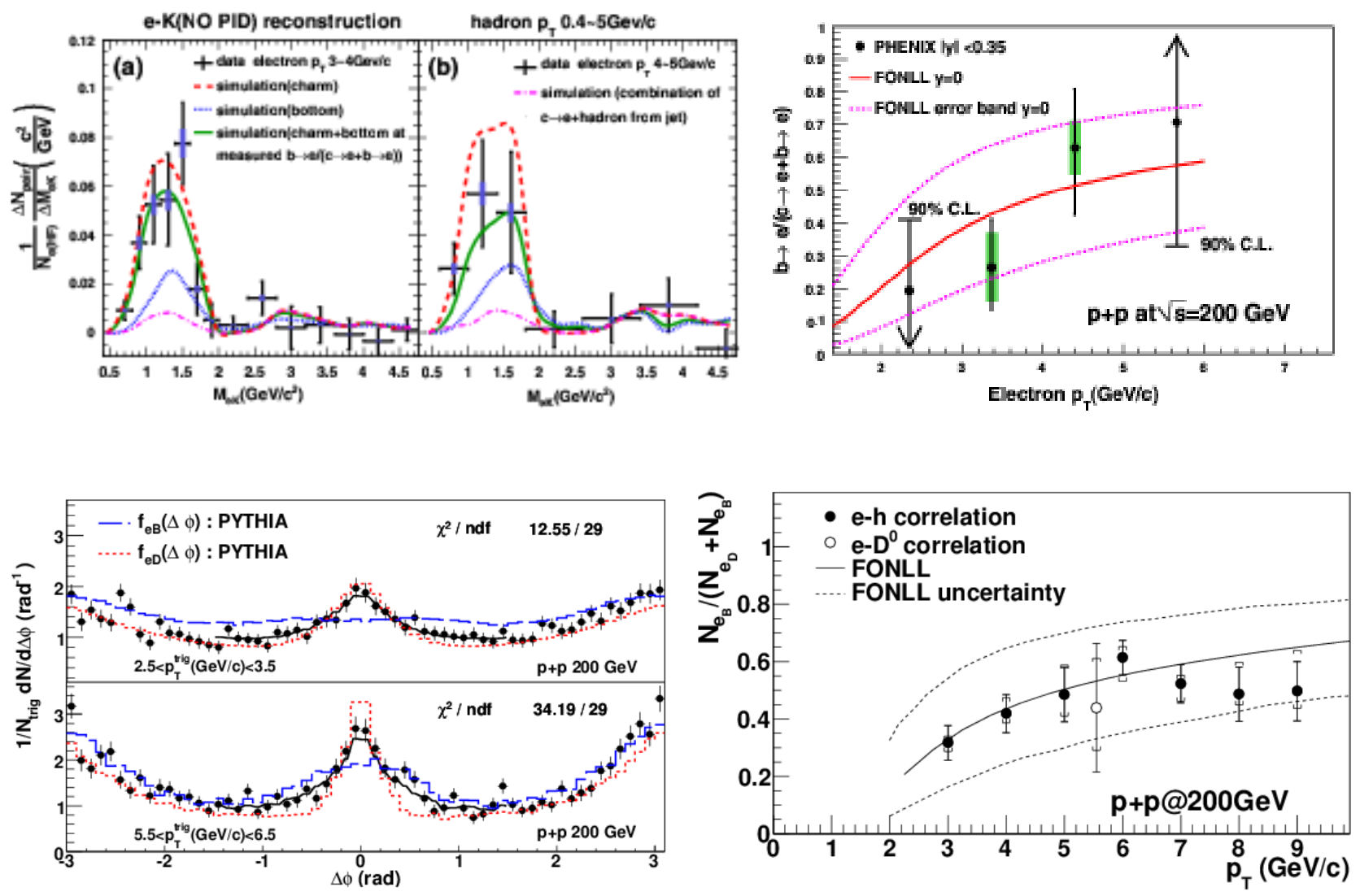

Figure 12: Statistical separation of the charm and bottom decay contributions to the electron sample from heavy-flavor hadron decays in pp collisions at $\sqrt{s}=200 \mathrm{GeV}$ with the PHENIX experiment (upper two panels) and with the STAR experiment (lower two panels). The invariant mass distribution of electron-hadron pairs measured with PHENIX is compared to charm and bottom decay templates obtained from simulations using PYTHIA and EVTGEN for two electron $p_{\mathrm{t}}$ ranges (upper left panel). The azimuthal angle between electrons from heavy-flavor decays and hadrons measured with STAR is compared to charm and bottom decay templates obtained with PYTHIA simulations for two electron $p_{\mathrm{t}}$ ranges (lower left panel). Relative contributions of bottom decays to the total yield of electrons from heavy-flavor hadron decays as functions of the electron $p_{\mathrm{t}}$ are compared to predictions from FONLL pQCD calculations (upper and lower left panel) (reprinted with permission from Refs. [163] (upper) and [175] (lower); Copyright $(2009,2010)$ by the American Physical Society). 
in two-particle correlation measurements increases dramatically with particle multiplicity, the statistical separation of electrons from charm and bottom decays was possible only in pp collisions at RHIC up to now.

In the PHENIX case, the extraction of the relative contributions from charm and bottom decays was based on the partial reconstruction of the decay $\mathrm{D} \rightarrow e^{+} \mathrm{K}^{-} X$ (and charge conjugate) [163]. The invariant-mass distribution of unlike charge-sign electron-hadron pairs exhibits a correlated signal below the D-meson mass because of the charge correlation in D-meson decays. The upper left panel of Fig. 12 shows this invariant-mass distribution normalized to the total yield of electrons from heavy-flavor decays in two hadron $p_{\mathrm{t}}$ bins. In this analysis, kaon identification was not performed but the mass of all reconstructed hadrons was assumed to be the kaon mass. Using a combination of the PYTHIA [120] and EVTGEN [176] simulation packages template distributions were generated that described the expected electron-hadron invariant-mass distributions from charm and bottom decays, shown as long-dashed and shortdashed lines, respectively, in the upper left panel of Fig. 12. A linear combination of these template distributions was fitted to the data with the relative contributions to the total distributions as the only fit parameter. The resulting contribution of bottom decays to the total electron spectrum from heavy-flavor hadron decays is shown in the upper right panel of Fig. 12 as a function of the electron $p_{\mathrm{t}}$. Below $3 \mathrm{GeV} / c$ and above $5 \mathrm{GeV} / c$ only upper and lower limits, respectively, of the relative bottom decay contribution could be measured. Nevertheless, a clear trend is observed that the bottom decay contribution increases with increasing electron $p_{\mathrm{t}}$. Moreover, the measured relative contributions are in good agreement with the expectation from a FONLL pQCD calculation [81, 82].

In the STAR case, the relative contributions from charm and bottom decays were separated statistically from each other via the measurement of angular correlations [175]. In a first approach, the distribution of the azimuthal angle $\Delta \phi$ between electrons from heavy-flavor decays and charged hadrons was studied. Such distributions, normalized to the number of electrons from heavy-flavor hadron decays, are shown for two electron $p_{\mathrm{t}}$ bins in the lower left panel of Fig. 12. The shapes of these distributions, in particular the width of the near side peak at $\Delta \phi=0$ is largely given by the decay kinematics of the charm and bottom hadron decays, where the larger mass of bottom hadrons leads to a broader peak compared to charm hadron decays. Template distributions reflecting the expected shapes of the electron-hadron azimuthal distributions as shown in the lower left panel of Fig. 12 were generated with PYTHIA [120]. The contribution of bottom decays relative to the sum of charm and bottom decays was obtained from a fit of the measured shape of the angular distribution using a linear combination of the charm and bottom templates with the relative normalization as fit parameter. A second, independent measurement of the contribution from bottom decays was conducted using the angular correlation of electrons from heavy-flavor hadron decays and $\mathrm{D}^{0}$ mesons reconstructed via their $\mathrm{K} \pi$ decay. Again, template distributions obtained via a PYTHIA [120] simulation were used to quantify the charm and bottom decay contributions. The observed away-side correlation signal in the electron- $\mathrm{D}^{0}$ azimuthal angle distribution was attributed to prompt charm hadron pair production $(\approx 75 \%)$ and bottom hadron decays $(\approx 25 \%)$, while the near side correlation signal was mainly due to bottom hadron decays only. The contributions of bottom decays to the total electron spectrum from heavy-flavor hadron decays as obtained with these two methods are shown in the lower right panel of Fig. 12 as functions of the electron $p_{\mathrm{t}}$. Because of the much larger solid angle coverage of the STAR apparatus compared to PHENIX, pair correlations could be measured with a much larger geometrical acceptance which is a clear advantage for such analyses. Results from the two techniques used in the STAR 

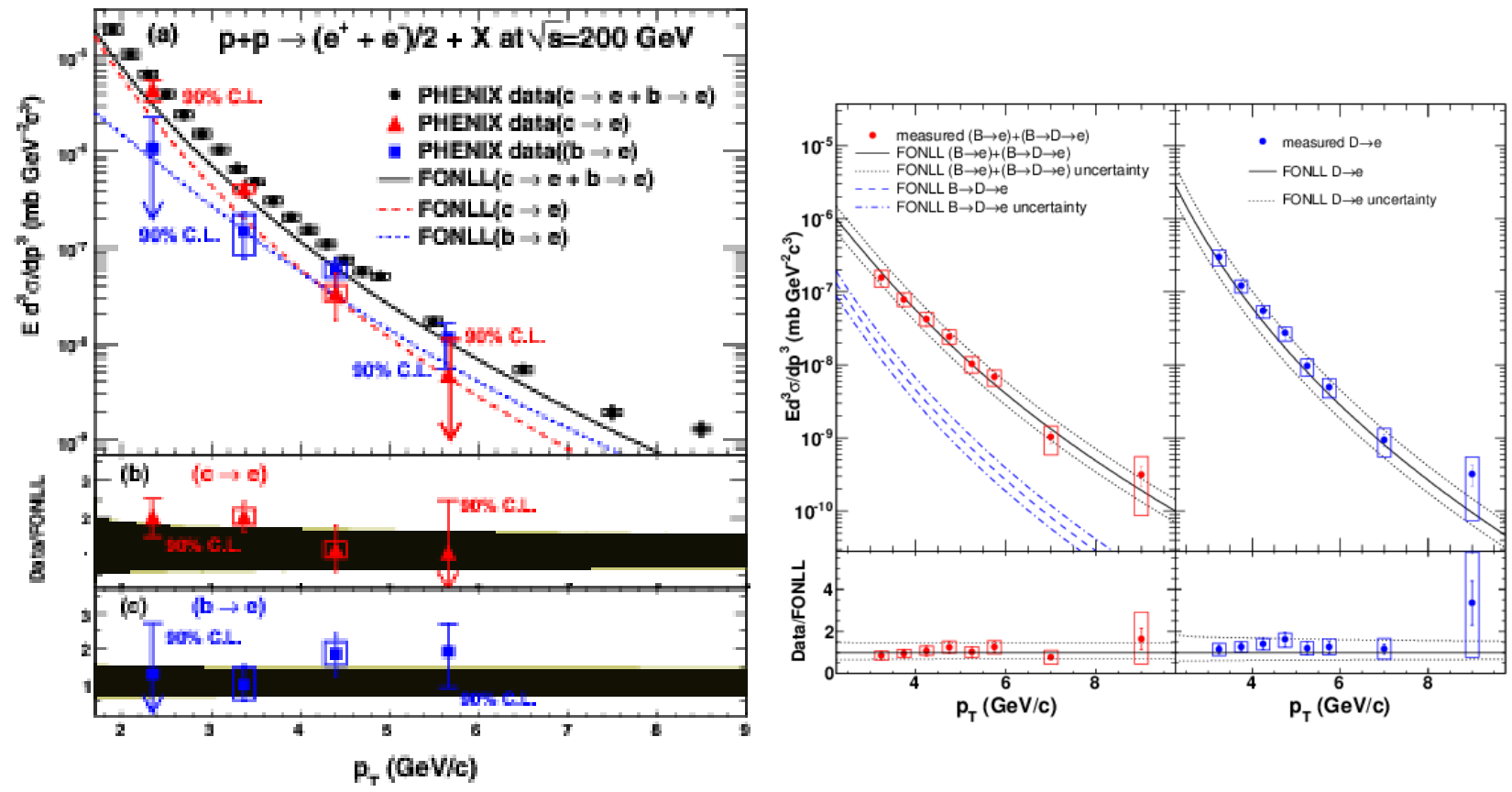

Figure 13: Invariant $p_{\mathrm{t}}$ differential production cross sections of electrons from charm and bottom hadron decays, respectively, as measured with the PHENIX (left panels) and STAR experiments (right panels) in pp collisions at $\sqrt{s}=200 \mathrm{GeV}$. Predictions from a FONLL pQCD calculation (shown as lines) are compared with the data on an absolute scale (upper panels) and in form of the ratio data/FONLL (lower panels) (reprinted with permission from Refs. [163] (left) and 175] (right); Copyright $(2009,2010)$ by the American Physical Society).

experiment agree with each other and with prediction from a FONLL pQCD calculation 81,82 as demonstrated in the lower right panel of Fig. 12.

From the measured invariant cross section of electrons from heavy-flavor hadron decays at $\sqrt{s}=200 \mathrm{GeV}$ (see Fig. 11) and the relative contributions from charm and bottom decays (see Fig. 12 it is straight forward to determine the electron production cross sections from charm and bottom hadron decays separately and compare them with the corresponding predictions from a FONLL pQCD calculation [81, 82]. The results from the PHENIX (STAR) experiments are shown in the left (right) panel of Fig. 13. For both cases FONLL is compared with the data on an absolute scale in the top panels while the ratio of the data to the calculation is shown in the lower panels. Given that FONLL provided a decent prediction for both the cross section of electrons from all heavy-flavor hadron decays and the for the relative contributions of charm and bottom decays to these cross sections it is not surprising that a good agreement between the FONLL predictions and the individual electron spectra from charm and bottom decays, respectively, was observed both with the PHENIX and STAR experiments.

Similar to the charm case (see above), the electron production cross sections from bottom decays were extrapolated to the full phase to determine the bottom production cross section. The extrapolation done for the STAR data was based on the PYTHIA generator [120] in the minimum bias mode for pp collisions and in an alternative, exclusive bottom production mode in which only leading order processes were included (see Ref. [175] for details). The total bottom production cross sections from these two approaches were $\sigma_{b \bar{b}}=1.34 \mu \mathrm{b}$ and $\sigma_{b \bar{b}}=1.83 \mu \mathrm{b}$, respectively 175. Corresponding extrapolations of the PHENIX data 163 using PYTHIA 


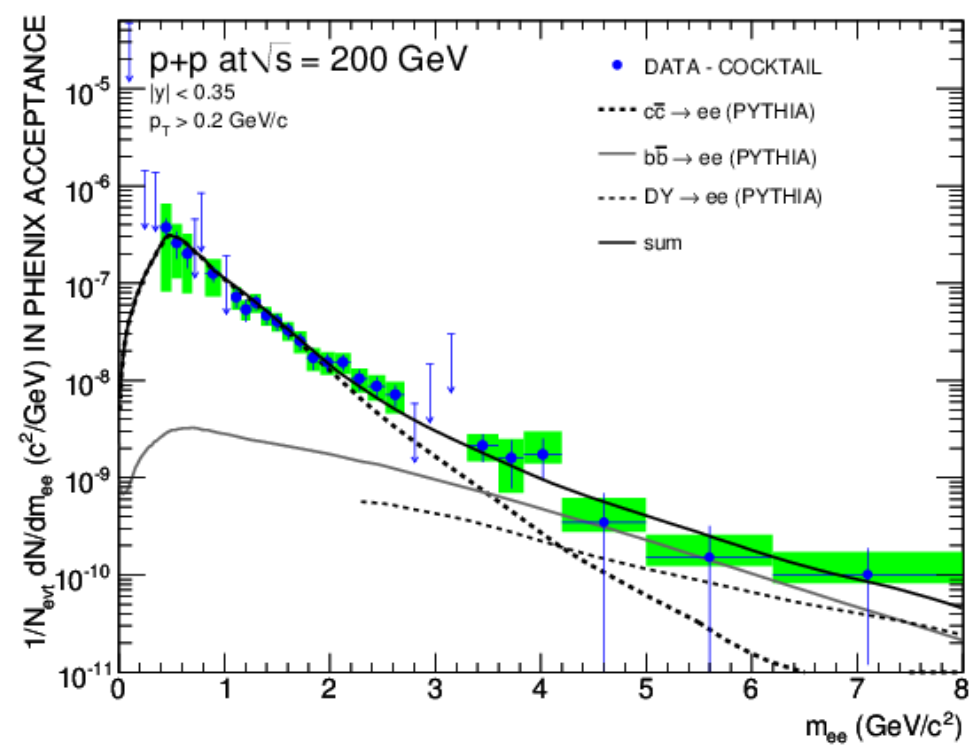

Figure 14: Electron-positron pair mass distribution from correlated decays of heavy-flavor hadrons measured in pp collisions at $\sqrt{s}=200 \mathrm{GeV}$ with the PHENIX experiment. Arrows indicate upper limits at 95\% confidence level where the systematic uncertainties were larger than the measured pair yields after background subtraction from other sources. Estimated contributions from charm decays (thick dashed line), bottom decays (thin solid line), pairs from the Drell-Yan process (thin dashed line), and the sum of these (thick solid line) are shown for comparison. See text for more details (reprinted from Ref. [164]).

and FONLL resulted in

$$
\begin{aligned}
\left.\frac{d \sigma_{b \bar{b}}}{d y}\right|_{y=0} & \left.=0.92_{-0.31}^{+0.34} \text { (stat. }\right)_{-0.36}^{+0.39} \text { (sys.) } \mu \mathrm{b} \\
\sigma_{b \bar{b}} & \left.=3.2_{-1.1}^{+1.2} \text { (stat. }\right)_{-1.3}^{+1.4} \text { (sys.) } \mu \mathrm{b} .
\end{aligned}
$$

Both the measurements with the STAR and PHENIX experiments are in agreement within uncertainties with the total bottom production cross section $\sigma_{b \bar{b}}=1.87_{-0.67}^{+0.99} \mu \mathrm{b}$ predicted with FONLL [81, 82]. To a large extent, the substantial uncertainties of the measurements were driven by the unavailable cross section measurements for electron production from bottom decay below an electron $p_{\mathrm{t}}$ of $3-4 \mathrm{GeV} / c$, indicating that future bottom production measurements have to include the low $p_{\mathrm{t}}$ region in order to provide a sensitive test of $\mathrm{pQCD}$ calculations over the full phase space.

In addition, with the PHENIX experiment the production of correlated electron-positron pairs from the decay of heavy-flavor hadron pairs from associated production in a hard scattering process was investigated in pp collisions at $\sqrt{s}=200 \mathrm{GeV}$ [164,165]. To statistically isolate such electron-positron pairs, in a first step the combinatorial background from uncorrelated background pairs was subtracted from the measured electron-positron pair mass distribution. The resulting signal distribution was corrected for the reconstruction efficiency. A pair cocktail from sources other than heavy-flavor hadron decays was calculated, similar to the single-electron case, and it was subtracted from the mass distribution of correlated electron-positron pairs. The remaining mass spectrum, shown in Fig. 14, was interpreted in terms of electron-positron pairs 
from correlated open heavy-flavor hadron decays. To statistically disentangle these contributions mass distribution templates were calculated with PYTHIA [120] which were then fitted to the data with the relative normalization as free parameters after the contribution from the Drell-Yan process was subtracted. As sources of systematic uncertainties in addition to the experimental uncertainties, the value of PYTHIA parameters such as the intrinsic $k_{\mathrm{t}}$ as well as the choice of the parton distribution function, and the uncertainty of the relative abundance of charm hadron species were considered. Furthermore, the dynamical correlations between heavy-flavor quark-antiquark pairs are not well known, which gave rise to additional systematic uncertainties. Within this approach, the total charm and bottom production cross sections were determined as

$$
\begin{aligned}
& \sigma_{c \bar{c}}=518 \pm 47 \text { (stat.) } \pm 135 \text { (sys.) } \pm 190(\text { model }) \mu \mathrm{b} \\
& \sigma_{b \bar{b}}=3.9 \pm 2.5(\text { stat. })_{-2}^{+3} \text { (sys.) } \mu \mathrm{b},
\end{aligned}
$$

where the model dependent uncertainty of the extrapolation to the full phase space is quoted separately for the charm cross section. Alternatively, the rapidity density of the charm production cross section at midrapidity and the total charm production cross section were determined after the bottom decay contribution was subtracted based on a bottom cross section of $3.7 \mu \mathrm{b}$ as obtained from a systematic study [177] of the total bottom production cross section as a function of $\sqrt{s}$ in pp collisions with PYTHIA [120]. The corresponding values are

$$
\begin{aligned}
\left.\frac{d \sigma_{c \bar{c}}}{d y}\right|_{y=0} & =118.1 \pm 8.4(\text { stat. }) \pm 30.7 \text { (sys. }) \pm 39.5(\text { model }) \mu \mathrm{b} \\
\sigma_{c \bar{c}} & =544 \pm 39(\text { stat. }) \pm 142(\text { sys. }) \pm 200(\text { model }) \mu \mathrm{b} .
\end{aligned}
$$

In summary, a consistent picture emerged from the measurement of electrons and correlated electron-positron pairs from open heavy-flavor hadron decays with the PHENIX and STAR experiments in pp collisions at $\sqrt{s}=200 \mathrm{GeV}$ at RHIC. The measured $p_{\mathrm{t}}$ differential single electron invariant cross sections are consistent with corresponding predictions from FONLL pQCD calculations. Also, such FONLL predictions are consistent with the total charm and bottom production cross sections obtained from single electron and electron-positron pair measurements with PHENIX and STAR at RHIC. These electron data provide a crucial reference for corresponding measurements in nucleus-nucleus collisions.

\subsubsection{Semimuonic heavy-flavor hadron decays}

The PHENIX experiment provides the unique opportunity at RHIC to study the rapidity dependence of open heavy-flavor production via the measurement of electrons at midrapidity (see above) and muons at forward rapidity from semileptonic decays of heavy-flavor hadrons. For the muon measurement it is crucial to subtract background from primary hadrons which penetrate all absorber layers and from other muon sources, i.e. from the light hadron decays before they reach the first hadron absorber material and from the decay of light hadrons that are produced inside the muon tracker volume of PHENIX. The techniques necessary for the background subtraction procedure were developed for the first measurement of muon production at forward pseudorapidity $(1.5 \leq|\eta| \leq 1.8)$ over the transverse momentum range $1 \leq p_{\mathrm{t}} \leq 3 \mathrm{GeV} / c$ in pp collisions at $\sqrt{s}=200 \mathrm{GeV}[166]$. The background from misidentified punchthrough hadrons 


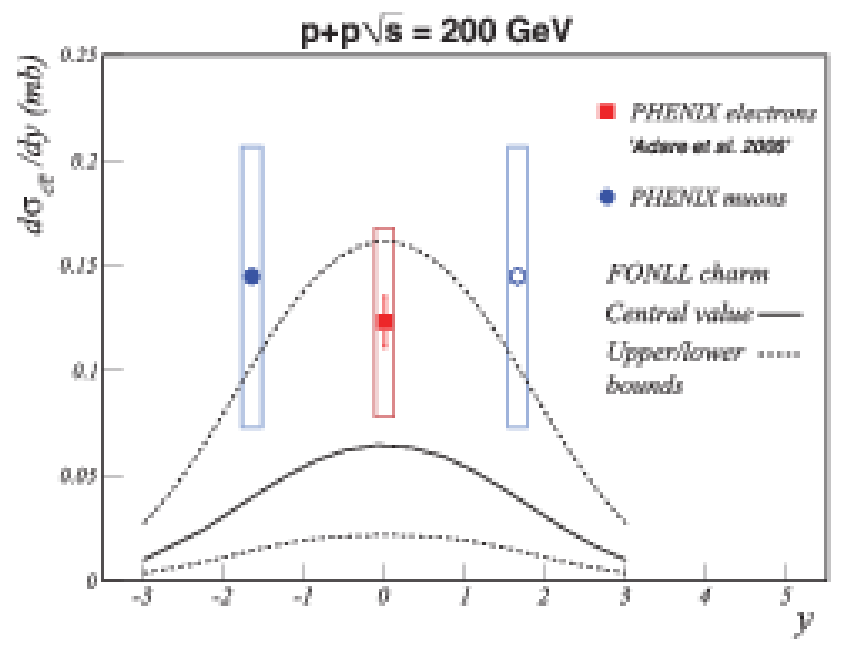

Figure 15: Charm production cross section as a function of rapidity in pp collisions at $\sqrt{s}=$ $200 \mathrm{GeV}$ measured with the PHENIX experiment using semileptonic open heavy-flavor hadron decays to electrons at midrapidity (closed square) and to muons at forward rapidity (closed circle). A prediction from FONLL pQCD calculation is shown for comparison (reprinted with permission from Ref. [156]; Copyright (2012) by the American Physical Society).

was significantly larger for positively charged compared to negatively charged muon candidates, mainly because of the relatively large nuclear absorption length for positively charged kaons in the absorber material. Therefore, only negatively charged muon candidates were considered for the further analysis. After the statistical background subtraction a muon excess remained which was attributed to semimuonic decays of open heavy-flavor hadrons, mainly D-mesons in the covered muon $p_{\mathrm{t}}$ range. A comparison of the $p_{\mathrm{t}}$ differential invariant cross section of the excess muons with corresponding calculations from PYTHIA and FONLL showed that the measured cross sections exceeded significantly both predictions [166]. The experimental systematic uncertainties, however, were huge and could not be quantified very precisely because of the lack of statistics available from the data set recorded during the 2001/2002 RHIC run.

With new data from the 2005 RHIC run the measurement could be improved significantly [156]. The production cross section of negatively charged muons from open heavy-flavor hadron decays was measured over an extended $p_{\mathrm{t}}$ range $\left(1 \leq p_{\mathrm{t}} \leq 7 \mathrm{GeV} / c\right)$ for rapidities $1.4<|y|<1.9$. The shape of the $p_{\mathrm{t}}$ differential invariant cross section predicted by a FONLL pQCD calculation agreed well with the measurement. Therefore, FONLL was used to extrapolate the measured spectrum to zero $p_{\mathrm{t}}$ and to calculate from the muon cross section a charm cross section at forward rapidity:

$$
\left.\left.\frac{d \sigma_{c \bar{c}}}{d y}\right|_{\langle y\rangle=1.65}=139 \pm 29 \text { (stat. }\right)_{-58}^{+51} \text { (sys.) } \mu \mathrm{b}
$$

The rapidity dependence of the charm production cross section as predicted from a FONLL pQCD calculation is compared to the cross section measured via decay electron at midrapidity and decay muons at forward rapidity in Fig. 15, where the combined muon result from both muon spectrometers is shown with a closed circle symbol at negative rapidity and reflected to forward rapidity (open circle). Within the substantial experimental and theoretical uncertainties the predicted cross sections are in agreement with the data. Clearly, more precise data 
Table 2: Rapidity densities $d \sigma_{\mathrm{q} \overline{\mathrm{q}}} / d y$ and total cross sections $\sigma_{\mathrm{q} \overline{\mathrm{q}}}$ for the production of heavy quark antiquark pairs q $\bar{q}$ (here, 'q' stands for either charm, 'c', or bottom, 'b') measured in various channels in pp collisions at $\sqrt{s}=200 \mathrm{GeV}$ at RHIC. In addition, the $p_{\mathrm{t}}$ and $y$ ranges covered by the measurements are given. Due to the limited $p_{\mathrm{t}}$ ranges, the measured yields have to be extrapolated to zero and high $p_{\mathrm{t}}$ to determine $d \sigma_{\mathrm{q} \overline{\mathrm{q}}} / d y$, and the measured fractions are listed. Errors quoted are the statistical (first values) and total systematic (second values) uncertainties.

\begin{tabular}{|c|c|c|c|c|}
\hline channel & $\begin{array}{l}\text { kinematic coverage } \\
\text { in } y \text { and } p_{\mathrm{t}}\end{array}$ & $\begin{array}{l}d \sigma_{\mathrm{q} \overline{\mathrm{q}}} / d y \\
\text { fraction measured } \\
\text { in covered } y \text { range }\end{array}$ & $\sigma_{\mathrm{q} \overline{\mathrm{q}}}$ & ref. \\
\hline $\mathrm{c} \overline{\mathrm{c}} \rightarrow \mathrm{D}^{0}, \mathrm{D}^{*}+\mathrm{X}$ & $\begin{array}{l}|y|<1 \\
0.6<p_{\mathrm{t}}<2.0 \mathrm{GeV} / c\left(\mathrm{D}^{0}\right) \\
2.0<p_{\mathrm{t}}<6.0 \mathrm{GeV} / c\left(\mathrm{D}^{*}\right)\end{array}$ & $\begin{array}{l}170 \pm 45_{-59}^{+38} \mu \mathrm{b} \\
\} \approx 67 \%\end{array}$ & $797 \pm 210_{-295}^{+208} \mu \mathrm{b}$ & 170 \\
\hline$c \bar{c} \rightarrow e^{ \pm}+X$ & $\begin{array}{l}|y|<0.35 \\
0.3<p_{\mathrm{t}}<9.0 \mathrm{GeV} / c\end{array}$ & $\begin{array}{l}119 \pm 12 \pm 38 \mu \mathrm{b} \\
\approx 55 \%\end{array}$ & $551 \pm 57 \pm 195 \mu \mathrm{b}$ & 155 \\
\hline $\mathrm{c} \overline{\mathrm{c}} \rightarrow \mathrm{e}^{+} \mathrm{e}^{-}+\mathrm{X}$ & $\begin{array}{l}|y|<0.35 \\
p_{t}^{e}>0.2 \mathrm{GeV} / c\end{array}$ & $\begin{array}{l}118.1 \pm 8.4 \pm 50.0 \mu \mathrm{b} \\
\approx 80 \%\end{array}$ & $544 \pm 39 \pm 245 \mu \mathrm{b}$ & 164 \\
\hline $\mathrm{c} \overline{\mathrm{c}} \rightarrow \mathrm{e}^{+} \mathrm{e}^{-}+\mathrm{X}$ & $\begin{array}{l}|y|<0.35 \\
p_{t}^{e}>0.2 \mathrm{GeV} / c\end{array}$ & $\begin{array}{l}\text { not quoted } \\
\approx 80 \%\end{array}$ & $518 \pm 47 \pm 233 \mu \mathrm{b}$ & 164 \\
\hline $\mathrm{c} \overline{\mathrm{c}} \rightarrow \mu^{-}+\mathrm{X}$ & $\begin{array}{l}1.4<|y|<1.9 \\
1<p_{\mathrm{t}}<7 \mathrm{GeV} / c\end{array}$ & $\begin{array}{l}139 \pm 29_{-58}^{+51} \mu \mathrm{b} \\
\approx 6 \%\end{array}$ & not quoted & 156 \\
\hline $\mathrm{b} \bar{b} \rightarrow e^{ \pm}+X$ & $\begin{array}{l}|y|<1 \\
3.0<p_{\mathrm{t}}<10.0 \mathrm{GeV} / c\end{array}$ & $\begin{array}{l}4.0 \pm 0.5 \pm 1.1 \mathrm{nb} \\
\approx 6.5 \%\end{array}$ & $1.34-1.83 \mu \mathrm{b}$ & 175 \\
\hline $\mathrm{b} \bar{b} \rightarrow \mathrm{e}^{ \pm}+\mathrm{X}$ & $\begin{array}{l}|y|<0.35 \\
3.0<p_{\mathrm{t}}<5.0 \mathrm{GeV} / c\end{array}$ & $\begin{array}{l}0.92_{-0.31-0.36}^{+0.34+0.39} \mu \mathrm{b} \\
\approx 5 \%\end{array}$ & $3.2_{-1.1-1.3}^{+1.2+1.4} \mu \mathrm{b}$ & $\mid 163$ \\
\hline $\mathrm{b} \overline{\mathrm{b}} \rightarrow \mathrm{e}^{+} \mathrm{e}^{-}+\mathrm{X}$ & $\begin{array}{l}|y|<0.35 \\
p_{t}^{e}>0.2 \mathrm{GeV} / c\end{array}$ & $\begin{array}{l}\text { not quoted } \\
\approx 99 \%\end{array}$ & $3.9 \pm 2.5_{-2}^{+3} \mu \mathrm{b}$ & 164 \\
\hline
\end{tabular}

are needed in order to sensibly discuss the shape of the rapidity distribution of charm in pp collisions at RHIC.

\subsubsection{Total heavy-flavor production cross sections at RHIC}

As discussed in this section heavy-flavor production has been studied in detail in pp collisions at RHIC in various channels. In addition to the spectral shapes of heavy-flavor observables, which are well described by NLO pQCD calculations, the rapidity densities $d \sigma_{\mathrm{c} \overline{\mathrm{c}}} / d y$ and $d \sigma_{\mathrm{b} \overline{\mathrm{b}}} / d y$ and the total heavy-flavor production cross sections $\sigma_{\mathrm{c} \overline{\mathrm{c}}}$ and $\sigma_{\mathrm{b} \overline{\mathrm{b}}}$ are the most relevant observables. The corresponding values measured in various channels are summarized in Tab. 2.

\subsection{Heavy flavor in $\mathrm{d}+\mathrm{Au}$ collisions}

Heavy-flavor observables are in agreement with predictions from FONLL pQCD calculations in pp collisions at RHIC, providing an experimental and theoretical baseline for correspond- 

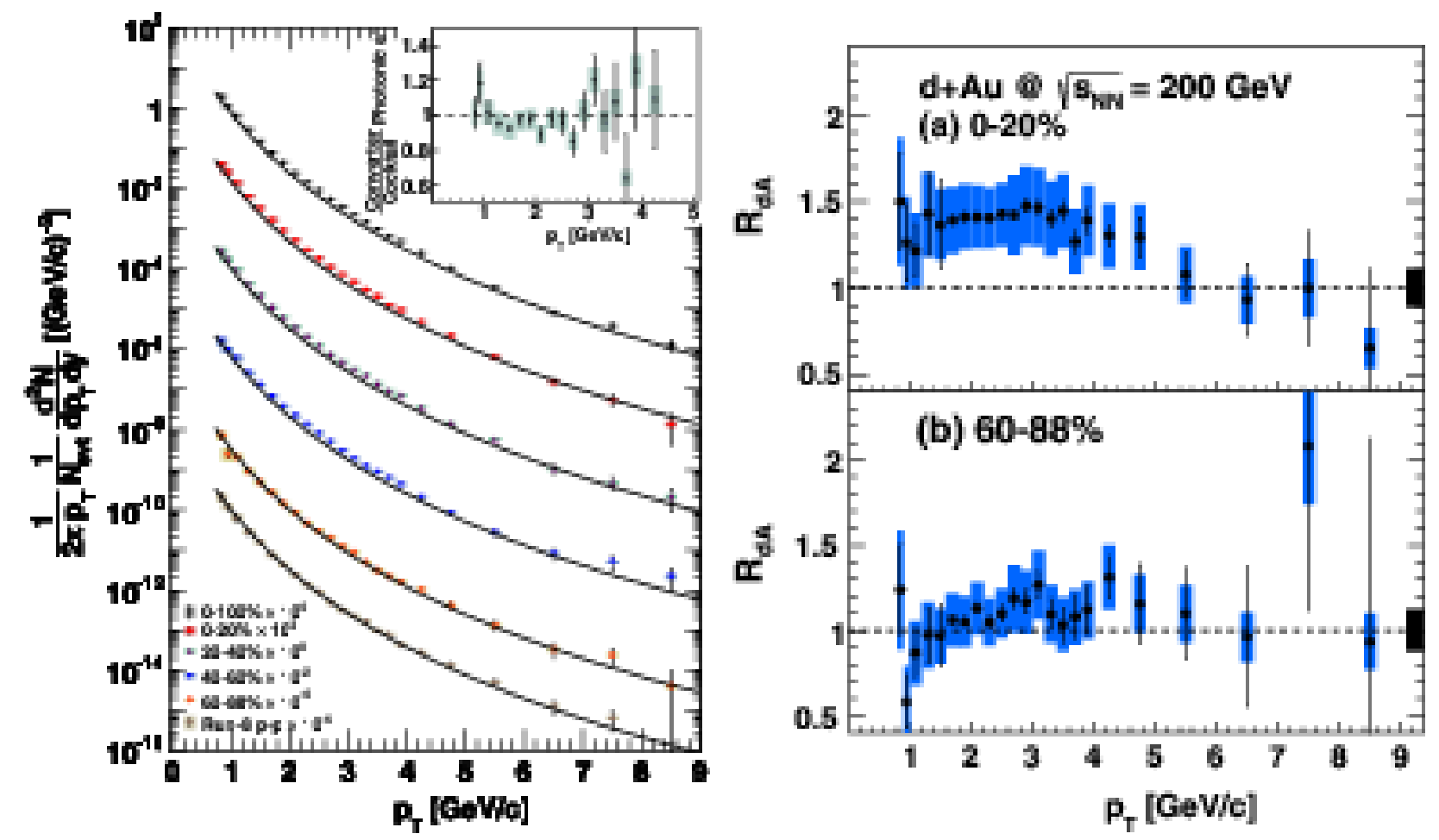

Figure 16: Electrons from heavy-flavor hadron decays in various centrality classes of $\mathrm{d}+\mathrm{Au}$ collisions and in pp collisions measured with the PHENIX experiment at $\sqrt{s_{\mathrm{NN}}}=200 \mathrm{GeV}$. The inset shows the ratio of electrons from heavy-flavor hadron decays measured via the converter and cocktail subtraction techniques, respectively (left panel). Nuclear modification factor $R_{\mathrm{dA}}$ of electrons from heavy-flavor hadron decays in the most central (a) and most peripheral (b) $\mathrm{d}+\mathrm{Au}$ centrality classes (right panel). See text for more details (reprinted with permission from Ref. [181]; Copyright (2012) by the American Physical Society).

ing measurements in collisions of heavy nuclei in which modifications of such observables are expected due to the interaction of heavy quarks with the produced hot and dense medium. However, in such collisions also cold nuclear matter effects play a role. Initial state effects, such as the modification of the parton distribution functions in nuclei (e.g. shadowing or saturation [178]), broadening of momentum distributions due to the scattering of incoming partons, Cronin enhancement [179, 180] of particle production at intermediate and high $p_{\mathrm{t}}$, and energy loss of partons in cold nuclear matter are expected to leave their footprint on open heavy-flavor observables. In collisions of heavy nuclei it is nearly impossible to cleanly disentangle cold matter effects from those induced by the presence of a hot and dense medium. Thus, it is necessary to investigate collisions of protons or deuterons with heavy nuclei. In such collisions the production of a hot and dense medium is not anticipated and, therefore, cold nuclear matter effects become accessible.

At RHIC, d+Au collisions were investigated first in an exploratory run in 2003. Data from this run firmly established [182 185] that for light hadrons in central $\mathrm{Au}+\mathrm{Au}$ collisions the strong suppression at high $p_{\mathrm{t}}$ relative to binary scaled pp collisions and the suppression of back-to-back azimuthal angular correlations is due to final-state interactions with the produced hot and dense medium and is not induced by cold nuclear matter effects. However, the inte- 
grated luminosity sampled in this run at RHIC was not sufficient for detailed measurements of open heavy-flavor observables. With the STAR experiment the production of $\mathrm{D}^{0}$ mesons was investigated [169 in the transverse momentum range $0.1<p_{\mathrm{t}}<3 \mathrm{GeV} / c$, and electrons from heavy-flavor hadron decays were measured in the range $1<p_{\mathrm{t}}<10 \mathrm{GeV} / c[173]$. Within large uncertainties the $\mathrm{d}+\mathrm{Au}$ data were consistent with data from pp collisions scaled by the number of binary collisions to account for the different collision geometry. The nuclear modification factor $R_{\mathrm{dA}}$ of electrons from open heavy-flavor hadron decays was observed to be consistent with a moderate Cronin enhancement. However, within substantial uncertainties the electron data were also consistent with an $R_{\mathrm{dA}}$ of one for all $p_{\mathrm{t}}$. Thus, no significant indication for cold nuclear matter effects on open heavy-flavor observables could be claimed based on the data from the 2003 run at RHIC.

The $2008 \mathrm{~d}+\mathrm{Au}$ run at RHIC constituted a major step forward in terms of the statistics available for analysis. For example, with the PHENIX experiment the sampled luminosity of $80 \mathrm{nb}^{-1}$ from the 2008 run corresponds to 30 times the sampled luminosity from the 2003 run. Using this data set the invariant $p_{\mathrm{t}}$ distribution of electrons from heavy-flavor hadron decays was measured in $\mathrm{d}+\mathrm{Au}$ collisions at $\sqrt{s_{\mathrm{NN}}}=200 \mathrm{GeV}$ in several centrality classes [181]. The collision centrality was determined event-by-event using the information from the PHENIX beam-beam counters. Within a Glauber MonteCarlo calculation the mean number of participants, $\left\langle N_{\text {part }}\right\rangle$, and the mean number of binary collisions, $\left\langle N_{\text {coll }}\right\rangle$, was determined for each $\mathrm{d}+\mathrm{Au}$ centrality class. Electrons were reconstructed at midrapidity $(|\eta|<0.35)$ in the transverse momentum range $0.85<p_{\mathrm{t}}<8.5 \mathrm{GeV} / c$. Background from sources other than open heavy-flavor hadron decays was statistically subtracted using the cocktail and converter subtraction techniques as discussed in section 3.2.3.

The resulting electron spectra from heavy-flavor hadron decays are shown in the left panel of Fig. 16 for minimum bias $\mathrm{d}+\mathrm{Au}$ collisions, for four $\mathrm{d}+\mathrm{Au}$ centrality classes, and for pp data recorded in the 2008 run at RHIC with the identical experimental setup as it was used for the $\mathrm{d}+\mathrm{Au}$ measurement. The lines represent fits to the previously published pp reference as discussed in section section 3.2 .3 , scaled by the appropriate number of binary collisions $N_{\text {coll }}$ for each $\mathrm{d}+\mathrm{Au}$ centrality classes. The pp data from the 2008 run of RHIC agree very well with the pp reference. To facilitate a direct comparison of the $\mathrm{d}+\mathrm{Au}$ electron spectra with the pp reference the nuclear modification factor $R_{\mathrm{dA}}$, defined as

$$
R_{\mathrm{dA}}=\frac{d N_{\mathrm{dA}}^{e} d p_{\mathrm{t}}}{\left\langle N_{\mathrm{coll}}\right\rangle \times d N_{\mathrm{pp}}^{e} d p_{\mathrm{t}}}
$$

was calculated for each $\mathrm{d}+\mathrm{Au}$ centrality class. $R_{\mathrm{dA}}$ is shown as a function of $p_{\mathrm{t}}$ for the most peripheral and the most central $\mathrm{d}+\mathrm{Au}$ centrality class in the right panel of Fig. 16. While in peripheral collisions $R_{\mathrm{dA}}$ is consistent with one within large systematic uncertainties, in central $\mathrm{d}+\mathrm{Au}$ collisions an enhancement of the yield of electrons from heavy-flavor hadron decays is observed up to a transverse momentum of $5 \mathrm{GeV} / c$. Measurements in pp collisions suggest that this $p_{\mathrm{t}}$ range is dominated by charm hadron decays (see section 3.2.3). Because the total heavy-flavor production cross section is expected to scale with the number of binary collisions, this enhancement at intermediate $p_{\mathrm{t}}$ can be interpreted in terms of a pronounced Cronin-like broadening [179] of the heavy-flavor hadron spectra while simultaneously keeping the integrated yield unchanged. A similar Cronin broadening was observed in the light quark sector in $\mathrm{d}+\mathrm{Au}$ collisions at RHIC, with the enhancement growing with hadron mass [186].

These electron data provide clear evidence that open heavy-flavor hadrons are subject to cold nuclear matter effects at RHIC. Consequently, such effects are expected to be present in 
the initial state of nucleus-nucleus collisions at RHIC. However, in the latter case the Croninlike enhancement will be convoluted with effects from the produced hot and dense medium such as partonic energy loss leading to a suppression of particle yields at high $p_{\mathrm{t}}$. While further experimental input from fully reconstructed open heavy-flavor hadrons and from a clean separation of charm and bottom observables is necessary to disentangle all relevant effects at RHIC, it is important to keep this first direct evidence for cold nuclear matter modifications of an open heavy-flavor observable in mind when interpreting data from nucleus-nucleus collisions at RHIC.

\subsection{Heavy flavor in Au+Au collisions}

\subsubsection{Hadronic heavy-flavor hadron decays}

The hot medium produced in heavy-ion collisions at RHIC is expected to leave its footprint on heavy-flavor observables because heavy quarks interact with this medium while propagating through it. In this context, detailed direct measurements of heavy-flavor hadrons in exclusive hadronic decay channels in nucleus-nucleus and the reference pp system are highly desirable. While $\mathrm{D}^{0}$ and $\mathrm{D}^{*+}$ measurements with the STAR experiment have become available for pp collisions at $\sqrt{s}=200 \mathrm{GeV}$ recently (see section 3.2.2), the situation is different for $\mathrm{Au}+\mathrm{Au}$ collisions at the same available energy per nucleon-nucleon pair. $\mathrm{D}^{0}$ mesons have been reconstructed with the STAR experiment at low $p_{\mathrm{t}}\left(p_{\mathrm{t}}<2 \mathrm{GeV} / c\right)$ in minimum bias $(0-80 \%$ central $)$ $\mathrm{Au}+\mathrm{Au}$ collisions via their $\mathrm{K} \pi$ decay [171]. Due to the unfavorably small ratio of the $\mathrm{D}^{0}$ signal to combinatorial background the extracted yield carried a systematic uncertainty of $40-50 \%$. The direct measurement of invariant $p_{\mathrm{t}}$ differential heavy-flavor hadron yields over an extended $p_{\mathrm{t}}$ range as a function of the nucleus-nucleus collision centrality will only be possible once data become available from the upgraded STAR and PHENIX experiments, which include silicon vertex spectrometers capable of separating secondary heavy-flavor hadron decay vertices from the primary collision vertex.

\subsubsection{Semielectronic heavy-flavor hadron decays}

The first measurement of open heavy-flavor production in nucleus-nucleus collisions [157] was conducted with the PHENIX experiment using data recorded during the very first run at RHIC. In $\mathrm{Au}+\mathrm{Au}$ collisions at $\sqrt{s_{\mathrm{NN}}}=130 \mathrm{GeV}$, electrons from semileptonic heavy-flavor hadron decays were statistically separated from electron background from other sources (mainly Dalitz decays of light mesons and photon conversions in material) using a cocktail subtraction technique as described above. The resulting electron $p_{\mathrm{t}}$ spectra from heavy-flavor hadron decays are shown in the left panel of Fig. 17 for minimum bias $(0-92 \%$ central $)$ and central $(0-10 \%$ central) $\mathrm{Au}+\mathrm{Au}$ collisions in the range $0.5<p_{\mathrm{t}}<3 \mathrm{GeV} / c$. A PYTHIA calculation [120] of the electron spectrum from heavy-flavor hadron decays in pp collisions at $\sqrt{s}=130 \mathrm{GeV}$ was scaled with the nuclear overlap integrals $T_{\mathrm{AA}}$ for minimum bias and central $\mathrm{Au}+\mathrm{Au}$ collisions, respectively, and is compared to the data in the left panel of Fig. 17. The PYTHIA parameters were tuned such that charm production data from fixed target experiments at the SPS and at FNAL as well as single electron data from the ISR were reproduced well. Within large uncertainties the scaled PYTHIA calculations agree with the measured electron yields, which in this rather low $p_{\mathrm{t}}$ range predominantly are due to charm hadron decays. This observation suggests that the total charm yield scales geometrically with the nuclear overlap integral or, equivalently, with the number of binary collisions when going from pp to $\mathrm{Au}+\mathrm{Au}$ collisions, as 

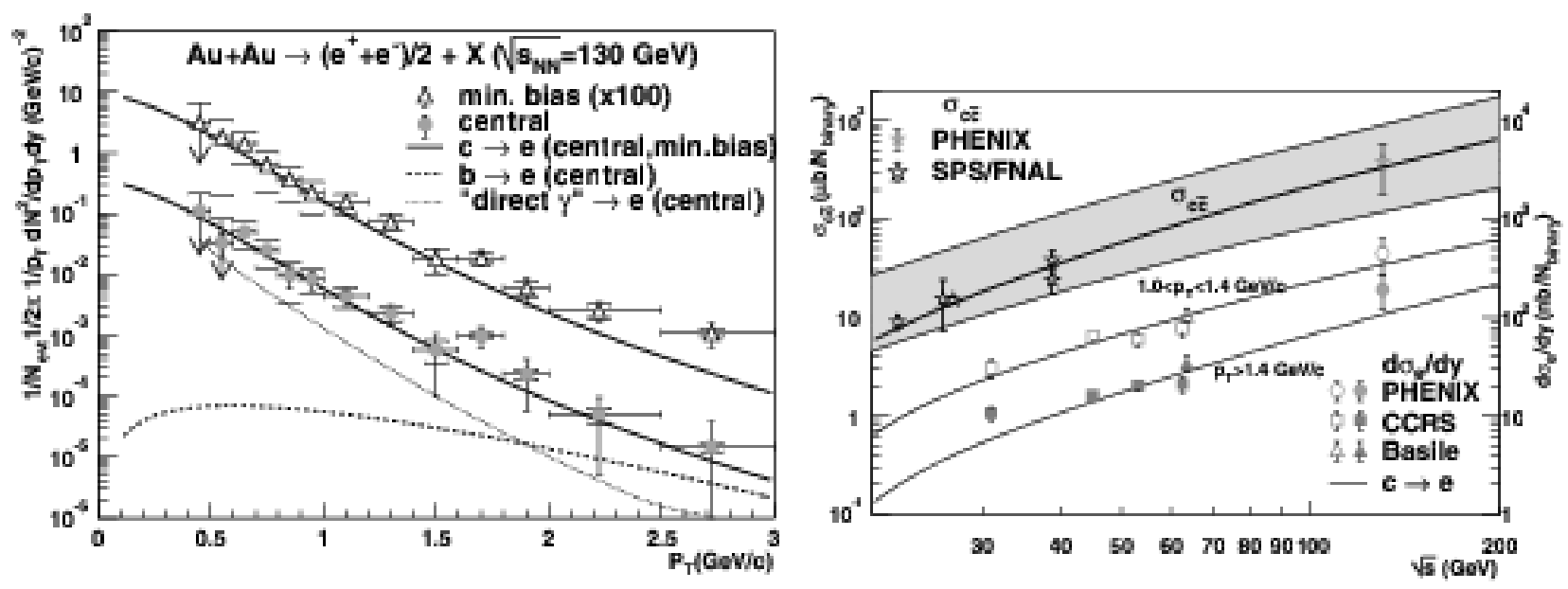

Figure 17: $p_{\mathrm{t}}$ differential yields of electrons from open heavy-flavor hadron decays measured with the PHENIX experiment in minimum bias and central $\mathrm{Au}+\mathrm{Au}$ collisions at $\sqrt{s_{\mathrm{NN}}}=130 \mathrm{GeV}$. For comparison corresponding PYTHIA calculations are shown (left panel). Electron production cross sections from heavy-flavor hadron decays from PHENIX and ISR measurements for the $p_{\mathrm{t}}$ ranges $1.0<p_{\mathrm{t}}<1.4 \mathrm{GeV} / c$ (open symbols) and $p_{\mathrm{t}}>1.4 \mathrm{GeV} / c$ (closed symbols), and the derived total charm production cross section per binary collision from PHENIX and other experiments as a function of $\sqrt{s}$. The solid lines and shaded band represent corresponding calculations from PYTHIA and an NLO pQCD calculation respectively (right panel). See text for more details (reprinted with permission from Ref. [157]; Copyright (2002) by the American Physical Society).

it is expected for hard probes such as heavy quarks. The measured yield of electrons with $p_{\mathrm{t}}>$ $0.8 \mathrm{GeV} / c$ at midrapidity corresponds to about $15 \%$ of the yield integrated over the full $p_{\mathrm{t}}$ range. Using the spectral shapes in $p_{\mathrm{t}}$ and $y$ as predicted from PYTHIA the total charm production cross section per binary collision was estimated. The latter is shown in the right panel of Fig. 17 together with corresponding results from ISR measurements 117,187 as a function of $\sqrt{s}$. In addition, the $\sqrt{s}$ dependence of rapidity densities per binary collision of heavy-flavor electron yields at midrapidity in two electron $p_{\mathrm{t}}$ intervals are depicted. For comparison, corresponding results from PYTHIA and from a HVQMNR NLO pQCD calculation [188 of the total charm production cross section are shown. Within substantial uncertainties the PYTHIA and NLO pQCD calculations are in agreement with the data.

While these electron data provided a first glimpse of open heavy-flavor physics in nucleusnucleus collisions, the limited precision of the measurement excluded any detailed conclusion concerning the centrality dependence of open heavy-flavor yields, not to speak of the $p_{\mathrm{t}}$ distributions of electrons from heavy-flavor hadron decays.

During the 2001 run period of RHIC a higher statistics electron data sample was recorded with reduced systematic uncertainties at midrapidity from $\mathrm{Au}+\mathrm{Au}$ collisions at $\sqrt{s_{\mathrm{NN}}}=200 \mathrm{GeV}$ with the PHENIX experiment. Electrons from heavy-flavor hadron decays were measured in the $p_{\mathrm{t}}$ range $0.4<p_{\mathrm{t}}<5.0 \mathrm{GeV} / c$, which is sensitive to charm production only. In a first step [158], these higher quality data allowed the measurement of the centrality dependence of the electron yields from heavy-flavor hadron decays in the $p_{\mathrm{t}}$ interval $0.4<p_{\mathrm{t}}<4.0 \mathrm{GeV} / c$. The background from other electron sources was subtracted using the converter subtraction 

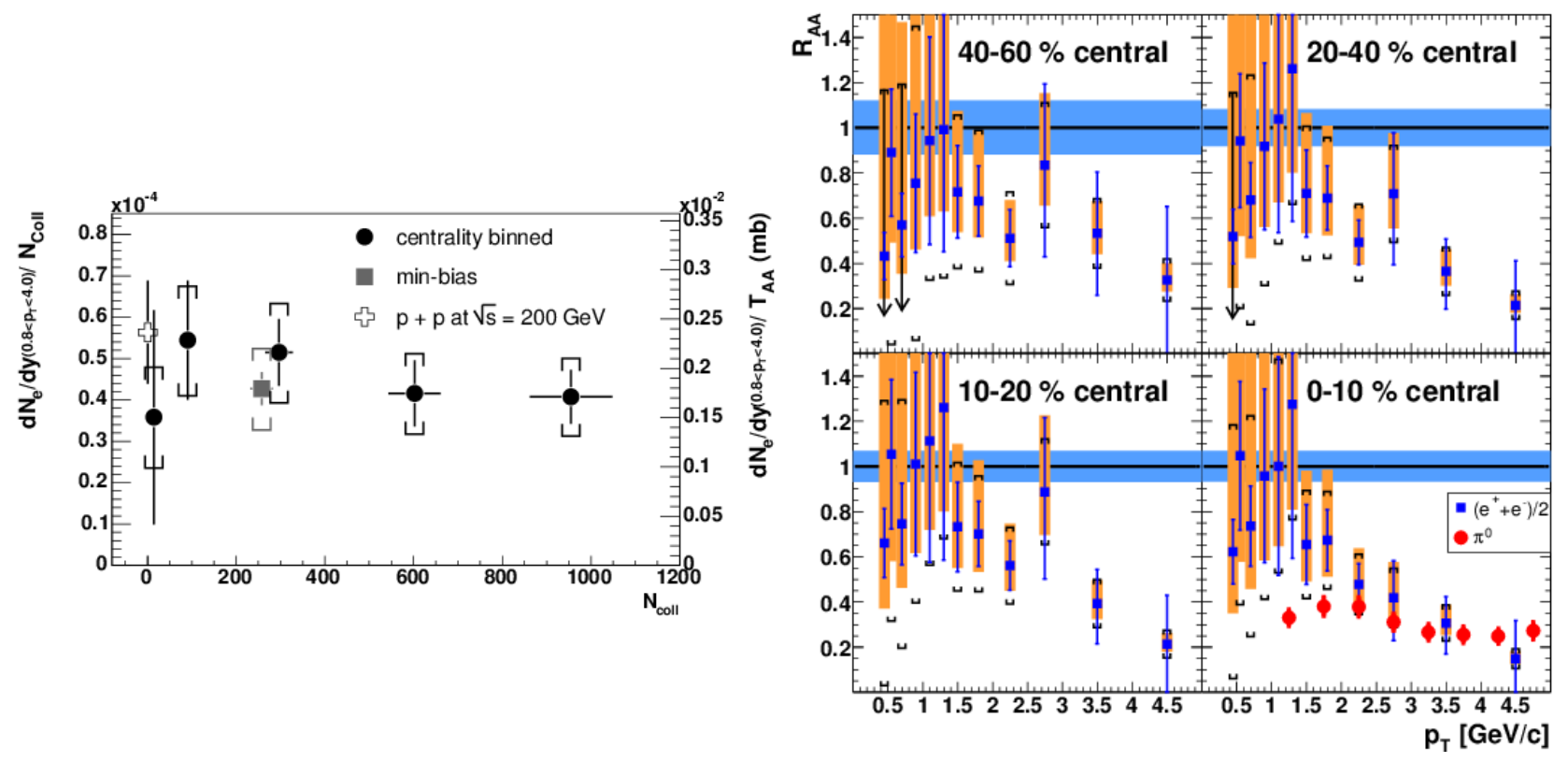

Figure 18: Electron yield (left axis) and cross section (right axis) from heavy-flavor hadron decays divided by the number of binary collisions as measured with the PHENIX experiment in $\mathrm{Au}+\mathrm{Au}$ collisions at $200 \mathrm{GeV}$ in the range $0.8<p_{\mathrm{t}}<4.0 \mathrm{GeV} / c$ as a function of the number of binary collisions, i.e. as function of collision centrality. For comparison the corresponding cross section from pp collisions is shown in addition (left panel). Nuclear modification factor $R_{\mathrm{AA}}$ of electrons from heavy-flavor hadron decays in four $\mathrm{Au}+\mathrm{Au}$ centrality classes at $\sqrt{s_{\mathrm{NN}}}=200 \mathrm{GeV}$ as a function of $p_{\mathrm{t}}$. Error bars (brackets) depict the statistical (systematic) errors from the $\mathrm{Au}+\mathrm{Au}$ measurement. Error boxes show the systematic errors from the pp reference, and the band around one indicates the relative systematic uncertainties of the nuclear overlap integrals $T_{\mathrm{AA}}$. For the most central collisions the neutral pion $R_{\mathrm{AA}}$ is shown for comparison (right panel) (reprinted with permission from Refs. [158] (left) and [160] (right); Copyright $(2005,2006)$ by the American Physical Society).

technique (see section 3.1.1 for details), which is superior to the cocktail subtraction technique at low $p_{\mathrm{t}}$ where the signal to background ratio is small. The resulting electron yields per binary collision are shown in the left panel of Fig. 18 as a function of the number of binary collisions, i.e. as a function of the $\mathrm{Au}+\mathrm{Au}$ collision centrality. Within the experimental uncertainties the electron yields, which are a measure of the number of produced $c \bar{c}$ pairs, scale with the number of binary collisions and in good agreement with the corresponding cross section measured in pp collisions at the same $\sqrt{s}$.

The total charm yield scales with the number of binary collisions consistent with pointlike production in a hard process. Final state interactions of charm quarks with the hot and dense medium produced in $\mathrm{Au}+\mathrm{Au}$ collisions at $\mathrm{RHIC}$ are not expected to have an effect on the total open charm yield. However, such effects will influence the momentum distribution of charm hadrons and, consequently, their decay products. Electron spectra from heavy-flavor hadron decays should be sensitive to such medium modifications for $p_{\mathrm{t}}>2.5 \mathrm{GeV} / c$. While the converter subtraction technique is well suited for the measurement of the total yield of electrons from heavy-flavor hadron decays (i.e. for low $p_{\mathrm{t}}$ electrons), it is of advantage to use the complementary cocktail subtraction technique for a precision measurement of the spectral 
shape towards larger electron $p_{\mathrm{t}}$.

Models utilizing radiative energy loss via induced gluon radiation as the relevant energy loss mechanism for heavy quarks in the hot and dense medium produced in $\mathrm{Au}+\mathrm{Au}$ collisions at RHIC predict only a moderate energy loss of charm and, in particular, bottom quarks at transverse momenta up to a few $\mathrm{GeV} / c$ [189,190]. In any case, due to the dead cone effect [98] the radiative energy loss of heavy quarks should be smaller than for light quarks. In view of this expectation, the discovery [160] of a substantial suppression of the electron yield from heavy-flavor hadron decays at intermediate and high $p_{\mathrm{t}}$ in central $\mathrm{Au}+\mathrm{Au}$ collisions relative to the binary collision scaled yields measured in pp collisions came as a complete surprise.

The right panel of Fig. 18 shows the nuclear modification factor $R_{\mathrm{AA}}$ as a function of $p_{\mathrm{t}}$ for electrons from heavy-flavor hadron decays in four $\mathrm{Au}+\mathrm{Au}$ centrality classes at $\sqrt{s_{\mathrm{NN}}}=$ $200 \mathrm{GeV}$ [160]. The pp reference was taken from the measurement described in Ref. [159]. Due to small signal to background ratio at low $p_{\mathrm{t}}$ the electrons yields at low $p_{\mathrm{t}}$ suffer from large systematic uncertainties which are reflected by the substantial uncertainties of $R_{\mathrm{AA}}$ for $p_{\mathrm{t}}<1.5 \mathrm{GeV} / c$. However, for all centrality classes $R_{\mathrm{AA}}$ is consistent with one in the low $p_{\mathrm{t}}$ region, which includes the dominant fraction of the total yields, in agreement with the observed scaling of the total charm yield with the number of binary collisions. Towards higher $p_{\mathrm{t}} \mathrm{a}$ suppression of electrons from heavy-flavor hadron decays develops which is most pronounced in the most central collisions at the highest $p_{\mathrm{t}}$. For comparison, the nuclear modification factor of neutral pions from [191] is shown for the case of the $10 \%$ most central $\mathrm{Au}+\mathrm{Au}$ collisions. For $p_{\mathrm{t}}>3 \mathrm{GeV} / c$ the nuclear modification factor of electrons from heavy-flavor hadron decays agrees quantitatively with the $R_{\mathrm{AA}}$ of neutral pions in central $\mathrm{Au}+\mathrm{Au}$ collisions at RHIC. This discovery presents a major challenge for models attempting to describe the partonic energy loss of heavy quarks traversing the medium created at RHIC.

Detailed model comparisons became possible with higher statistics electron data from heavy-flavor hadron decays recorded in $\mathrm{Au}+\mathrm{Au}$ collisions at $\sqrt{s_{\mathrm{NN}}}=200 \mathrm{GeV}$ with the PHENIX 155, 162] and STAR experiments [173] in the 2004 run at RHIC. With the STAR experiment electrons were measured in this run in the transverse momentum range $1.2<p_{\mathrm{t}}<$ $10 \mathrm{GeV} / c$. Electron background from photonic sources, i.e. light meson Dalitz decays and electrons from photon conversions in material, was measured via an invariant mass technique. Remaining background from other sources was subtracted using a cocktail approach. The left panel of Fig. 19 depicts the resulting heavy-flavor electron spectra measured with the STAR experiment in three $\mathrm{Au}+\mathrm{Au}$ centrality classes, $\mathrm{d}+\mathrm{Au}$ collisions, and the reference pp system 173. For comparison results from FONLL pQCD calculations 81,82 scaled to the pp measurement are shown, which indicate that up to $3 \mathrm{GeV} / c$ electrons originate mainly from charm hadron decays while towards higher $p_{\mathrm{t}}$ bottom hadron decays become increasingly more important. With the PHENIX experiment electrons have been measured in the transverse momentum range $0.3<p_{\mathrm{t}}<9.0 \mathrm{GeV} / c$ at midrapidity $(|y|<0.35)$. Electron background from other sources was subtracted statistically using the converter method for $p_{\mathrm{t}}<1.6 \mathrm{GeV} / c$ and the cocktail method for higher $p_{\mathrm{t}}$. The resulting electron spectra from heavy-flavor hadron decays for various $\mathrm{Au}+\mathrm{Au}$ centrality classes $[155,162$ are shown in the right panel of Fig. 19 together with the corresponding measurement from pp collisions [161]. The solid lines depict the line shape from a FONLL calculation [81,82 normalized to the pp data and scaled with the number of binary collisions for each $\mathrm{Au}+\mathrm{Au}$ centrality class.

While the binary collision scaled pp reference agrees with the electron yields from heavyflavor hadron decays at low $p_{\mathrm{t}}$ for all $\mathrm{Au}+\mathrm{Au}$ centrality classes this is different at high $p_{\mathrm{t}}$ where the heavy-flavor electron yields in $\mathrm{Au}+\mathrm{Au}$ are suppressed relative to the scaled pp reference. 

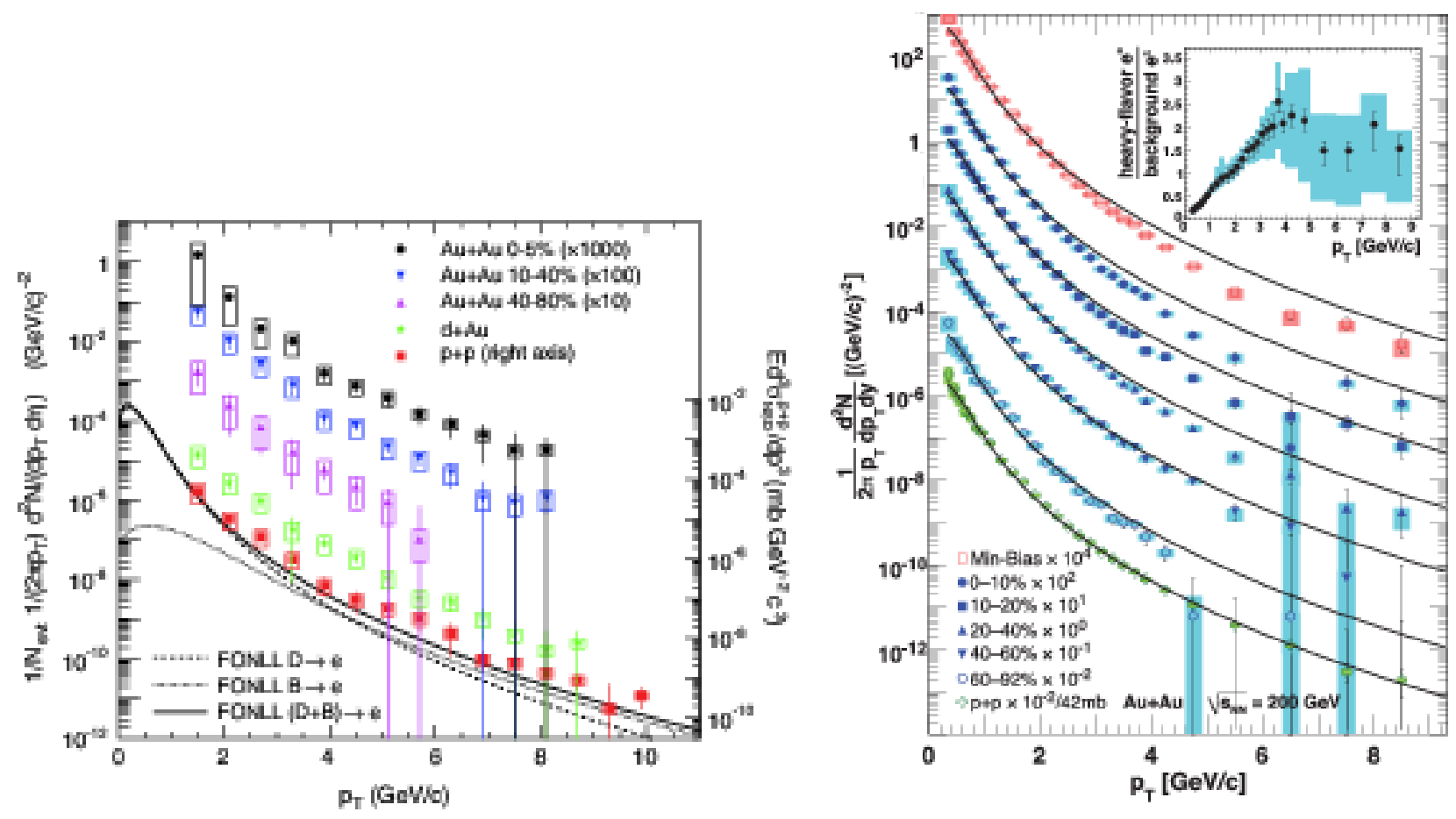

Figure 19: Electron $p_{\mathrm{t}}$ spectra from heavy-flavor hadron decays measured with the STAR experiment in $\mathrm{pp}, \mathrm{d}+\mathrm{Au}$, and various $\mathrm{Au}+\mathrm{Au}$ centrality classes at $\sqrt{s_{\mathrm{NN}}}=200 \mathrm{GeV}$. FONLL predictions [81,82] are compared with the data for the pp case, for which the right axis indicates the corresponding cross section values (left panel). Invariant $p_{\mathrm{t}}$ differential distributions of electrons from heavy-flavor hadron decays measured with the PHENIX experiment for various $\mathrm{Au}+\mathrm{Au}$ centrality classes and for pp collisions. The solid lines are a from a FONLL calculation and the inset shows the signal to background ratio for minimum bias $\mathrm{Au}+\mathrm{Au}$ collisions (right panel) (reprinted with permission from Refs. [173] (left) and [155] (right); Copyright (2007, 2011) by the American Physical Society).

The suppression is more pronounced in central collisions compared to more peripheral centrality classes. This behavior is quantified in the left panel of Fig. 20 which shows the nuclear modification factor $R_{\mathrm{AA}}$ of electrons from heavy-flavor hadron decays for two $p_{\mathrm{t}}$ ranges as a function of the number of participating nucleons, $N_{\text {part }}$, in $\mathrm{Au}+\mathrm{Au}$ collisions $[162$. For the range $p_{\mathrm{t}}>0.3 \mathrm{GeV} / c$, which includes more than $50 \%$ of the total electron yield from heavyflavor hadron decays, $R_{\mathrm{AA}}$ is consistent with one, confirming the binary collision scaling of the total heavy-flavor hadron yield in $\mathrm{Au}+\mathrm{Au}$ collisions at $\mathrm{RHIC}$. For the range $p_{\mathrm{t}}>3 \mathrm{GeV} / c, R_{\mathrm{AA}}$ decreases with increasing centrality reaching a value of $\approx 0.4$ for the $10 \%$ most central $\mathrm{Au}+\mathrm{Au}$ collisions at $\sqrt{s_{\mathrm{NN}}}=200 \mathrm{GeV}$. The suppression of electrons from heavy-flavor hadron decays can not be compared directly to light hadron suppression in the same $p_{\mathrm{t}}$ range. However, electrons from charm hadron decays with $p_{\mathrm{t}}>3 \mathrm{GeV} / c$ originate to a large extent from the decay of $\mathrm{D}$ mesons with $p_{\mathrm{t}}>4 \mathrm{GeV} / c$ [120]. Therefore, the electron $R_{\mathrm{AA}}$ above $3 \mathrm{GeV} / c$ is compared with the neutral pion $R_{\mathrm{AA}}$ above $4 \mathrm{GeV} / c$ [191]. In this intermediate $p_{\mathrm{t}}$ range the trend visible in the left panel of Fig. 20 indicates a smaller suppression of heavy-flavor hadrons relative to light-flavor mesons in the hot and dense medium produced in $\mathrm{Au}+\mathrm{Au}$ collisions at RHIC. However, for a firm conclusion it would be necessary to consider also cold nuclear matter effects, 

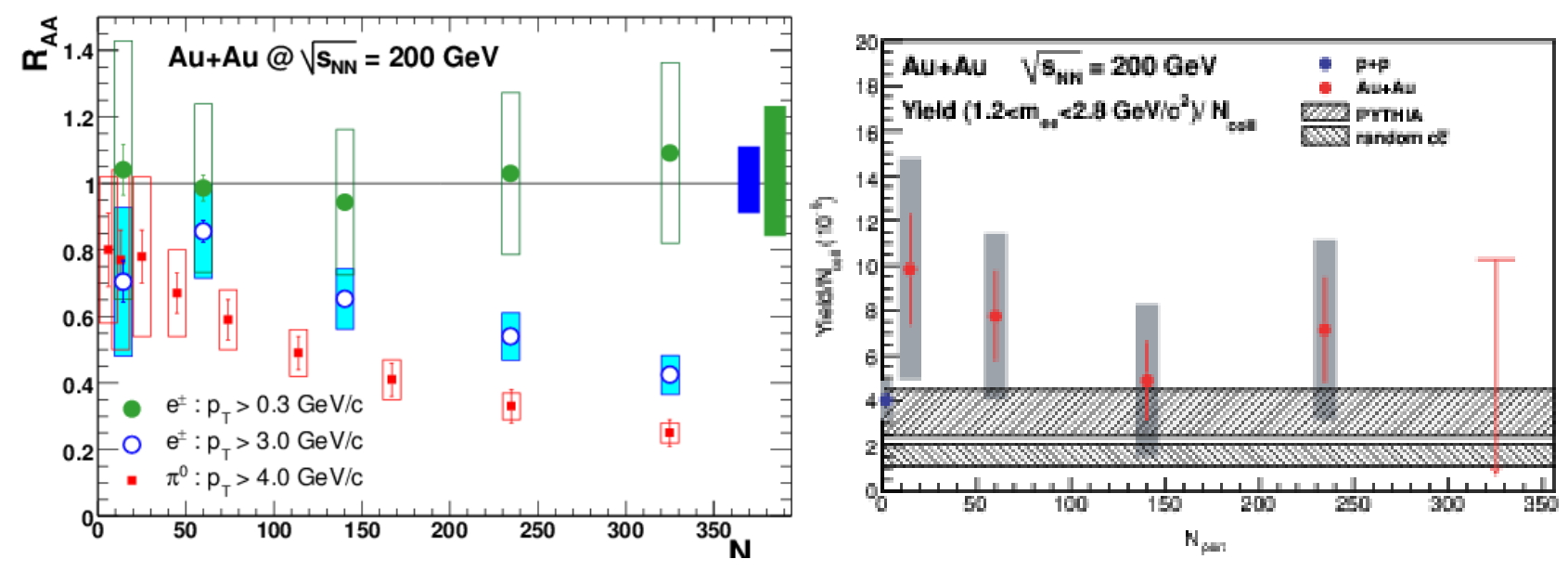

Figure 20: Nuclear modification factor $R_{\mathrm{AA}}$ of electrons from heavy-flavor hadron decays measured with the PHENIX experiment in $\mathrm{Au}+\mathrm{Au}$ collisions at $\sqrt{s_{\mathrm{NN}}}=200 \mathrm{GeV}$ as a function of the collision centrality (quantified by the number of participants, $N_{\text {part }}$ ) for the electron transverse momentum ranges $p_{\mathrm{t}}>0.3 \mathrm{GeV} / c$ and $p_{\mathrm{t}}>3.0 \mathrm{GeV} / c$, respectively. The boxes at $R_{\mathrm{AA}}=1$ indicate the common relative uncertainty from the pp reference for $p_{\mathrm{t}}>0.3 \mathrm{GeV} / c$ (right box) and $p_{\mathrm{t}}>3.0 \mathrm{GeV} / c$ (left box). $R_{\mathrm{AA}}$ of neutral pions with $p_{\mathrm{t}}>4.0 \mathrm{GeV} / c[191$ is shown for comparison (left panel). Dielectron yield in the intermediate mass range $1.2<m_{\mathrm{ee}}<2.8 \mathrm{GeV} / c^{2}$ per binary collision collision as a function of $N_{\text {part }}$ in comparison with two bands corresponding to different estimates of the contribution from charm hadron decays. See text for more details (right panel) (reprinted with permission from Refs. [162 (left) and [165] (right); Copyright $(2007,2010)$ by the American Physical Society).

which might be different for light and heavy-flavor hadrons and which have been measured with limited precision only up to now [173,181]. Furthermore, the interpretation of electron spectra from heavy-flavor hadron decays in $\mathrm{Au}+\mathrm{Au}$ collisions is not straight forward because of the decay kinematics involved and, most important, because the relative contributions from charm and bottom hadron decays can be different from those measured with substantial uncertainties in pp collisions at the same energy [163, 175.

A similar general picture emerges from the centrality dependence of dielectron yields per binary collision measured with the PHENIX experiment 165 in the intermediate mass range $1.2<m_{\mathrm{ee}}<2.8 \mathrm{GeV} / c^{2}$ as shown in the right panel of Fig. 20 . This mass region is dominantly populated by dielectrons from correlated charm hadron decays. The measured yields are compared with two extreme scenarios concerning the correlation between the original charm quark and antiquark. In the first approach, a PYTHIA calculation for pp collisions [120] is simply scaled with the number of binary collisions to the $\mathrm{Au}+\mathrm{Au}$ case, thus keeping the $c \bar{c}$ correlation intact, i.e. unmodified by the interaction with the hot and dense medium in $\mathrm{Au}+\mathrm{Au}$ collisions. In the second approach, the kinematic $c \bar{c}$ correlation is assumed to be completely destroyed by the interaction with the medium, i.e. the electrons and positrons are sampled randomly from the measured electron spectra from heavy-flavor hadron decays [162]. This approach gives rise to a considerable softening of the dielectron mass spectra resulting in a reduced dielectron yield in the intermediate mass region compared to the PYTHIA scaling as indicated in the right panel of Fig. 20. In view of the suppression of the electron yields from heavy-flavor hadron decays at high $p_{\mathrm{t}}$ the observed binary collision scaling of the intermediate mass dielectron yield 


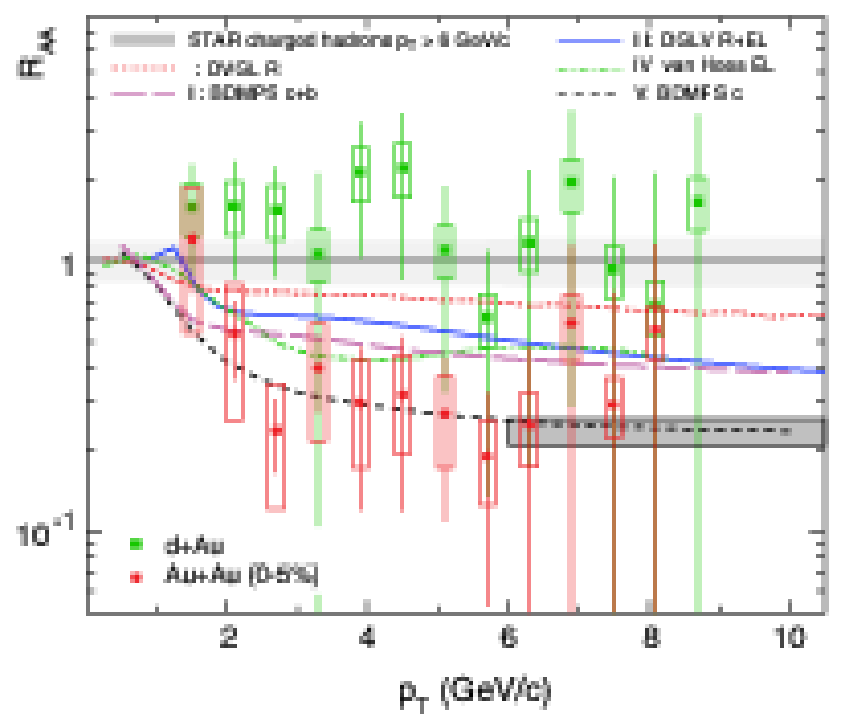

Figure 21: Nuclear modification factor $R_{\mathrm{AA}}$ of electrons from heavy-flavor hadron decays measured as a function of $p_{\mathrm{t}}$ with the STAR experiment in $\mathrm{d}+\mathrm{Au}$ and in the $5 \%$ most central $\mathrm{Au}+\mathrm{Au}$ collisions at $\sqrt{s_{\mathrm{NN}}}=200 \mathrm{GeV}$. For comparison the corresponding $R_{\mathrm{AA}}$ of charged hadrons 192 is indicated as well as results from various theoretical model calculations. See text for more details (reprinted with permission from Ref. [173]; Copyright (2007) by the American Physical Society).

for all collisions centralities might point to the cancellation of two competing effects. With increasing centrality the energy loss of charm increases, resulting in a softening of the dielectron mass spectra and, consequently, in a reduced dielectron yield in the intermediate mass region. This might be compensated by an increasing yield of dielectrons from thermal radiation with centrality, which was observed previously with the NA60 experiment [127, 128] at the CERN SPS (see section 2.1).

In order to address the suppression of electrons from heavy-flavor hadron decays quantitatively and to relate this with the energy loss of heavy quarks traversing the hot and dense medium produced in central $\mathrm{Au}+\mathrm{Au}$ collisions at $\mathrm{RHIC}$, model calculations of the nuclear modification factor $R_{\mathrm{AA}}\left(p_{\mathrm{t}}\right)$ from various theoretical approaches are confronted with electron data from the most central $\mathrm{Au}+\mathrm{Au}$ collisions.

Fig. 21 shows such a comparison for the case of the STAR experiment [173]. The nuclear modification factor of electrons from heavy-flavor hadron decays in $\mathrm{d}+\mathrm{Au}$ collisions is consistent with a moderate Cronin enhancement albeit with large uncertainties. For the $5 \%$ most central $\mathrm{Au}+\mathrm{Au}$ collisions predictions of the heavy-flavor electron $R_{\mathrm{AA}}$ are shown from five different model calculations. Curve I corresponds to radiative energy loss via a few hard scattering processes in the medium calculated within the DGLV formalism [189], where an initial gluon density of $d N_{\mathrm{g}} / d y=1000$ was assumed, consistent with hadron suppression in the light quark sector. Another radiative energy loss calculation, employing multiple soft collisions within the BDMPS formalism [190] is shown as curve II, where the maximum transport coefficient $\hat{q}=14 \mathrm{GeV}^{2} / \mathrm{fm}$ was used which is still consistent with light hadron suppression. Both radiative energy loss calculations predict less suppression than is observed. Adding collisional, i.e. elastic, energy loss to a DGLV-based calculation and introducing path length fluctuations [94] leads to 


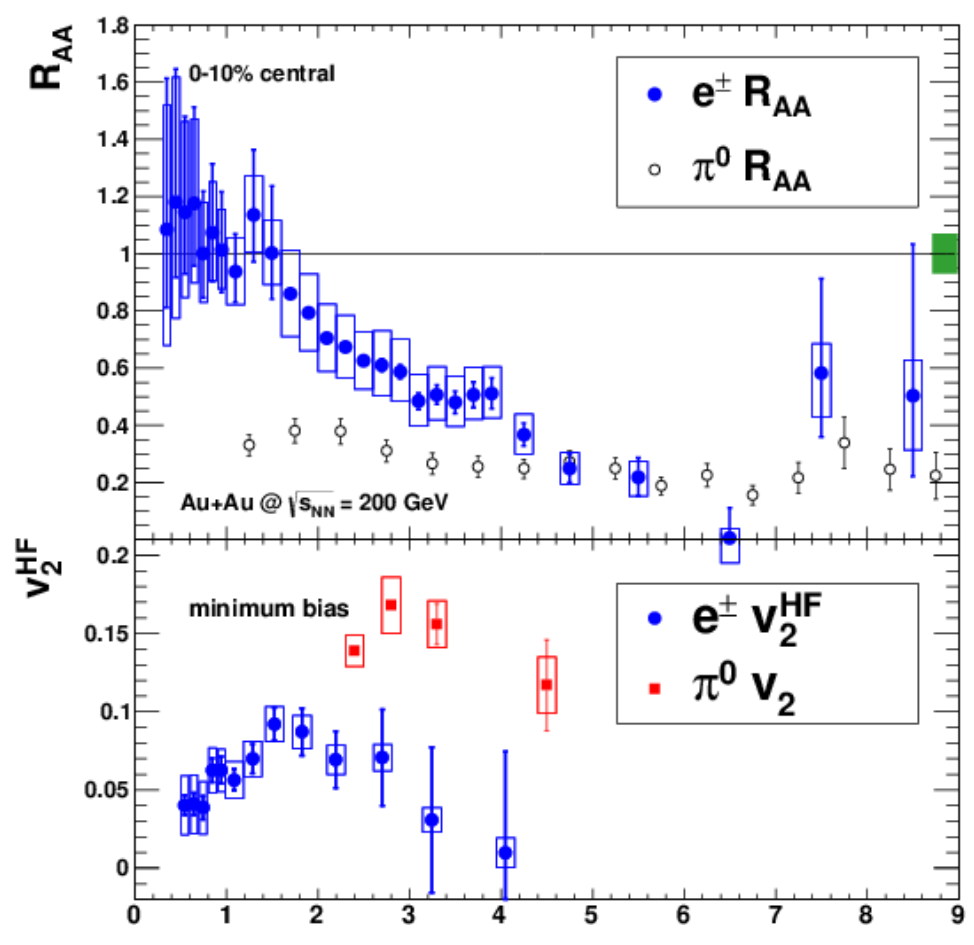

Figure 22: $R_{\mathrm{AA}}$ of electrons from heavy-flavor hadron decays measured as a function of $p_{\mathrm{t}}$ with the PHENIX experiment in the $10 \%$ most central $\mathrm{Au}+\mathrm{Au}$ collisions at $200 \mathrm{GeV}$ [155] in comparison with the corresponding neutral pion $R_{\mathrm{AA}}$ from Ref. 191 (upper panel). Elliptic flow magnitude $v_{2}$ of electrons from heavy-flavor hadron decays 155 and of neutral pions 193 in minimum bias $\mathrm{Au}+\mathrm{Au}$ collisions (lower panel).

a larger, but still not sufficient suppression as demonstrated by curve III. In a complementary approach (curve IV) heavy-quark energy loss is implemented via elastic scattering involving charm and bottom resonance excitations in the medium as well as leading order t-channel gluon exchange [101]. In particular towards high $p_{\mathrm{t}}$, where the contribution from bottom hadron decays is expected to become important heavy-quark energy loss models consistently seem to underpredict the observed suppression. However, given the relatively large uncertainties of the STAR electron data it is difficult to draw definite conclusions going beyond the general statement that in central $\mathrm{Au}+\mathrm{Au}$ collisions the yield of electrons from heavy-flavor hadron decays is strongly suppressed at high $p_{\mathrm{t}}$, consistent with substantial energy loss of heavy quarks in the hot and dense medium produced at RHIC.

The PHENIX electron data from heavy-flavor hadron decays provide a higher precision and, therefore, allow a better discrimination between different theoretical model calculations [155]. The nuclear modification factor $R_{\mathrm{AA}}$ of electrons from heavy-flavor hadron decays, shown as a function of $p_{\mathrm{t}}$ for the $10 \%$ most central $\mathrm{Au}+\mathrm{Au}$ collisions in the upper panel of Fig. 22, is larger compared to the neutral pion $R_{\mathrm{AA}}[191]$ at low and intermediate $p_{\mathrm{t}}$ but towards high $p_{\mathrm{t}}$ the suppression of the two particle species is of similar size. Further constraints are provided by the magnitude $v_{2}$ of the elliptic flow of electrons from heavy-flavor hadron decays as compared for minimum bias $\mathrm{Au}+\mathrm{Au}$ collisions to the corresponding neutral pion $v_{2}[193]$ in the lower panel of Fig. 22. Although for $p_{\mathrm{t}}$ above $2 \mathrm{GeV} / c$ the $\pi^{0} v_{2}$ is larger than the $v_{2}$ of electrons from heavyflavor decays the latter is clearly larger than zero up to a $p_{\mathrm{t}}$ of $\approx 3 \mathrm{GeV} / c$. The suppression 

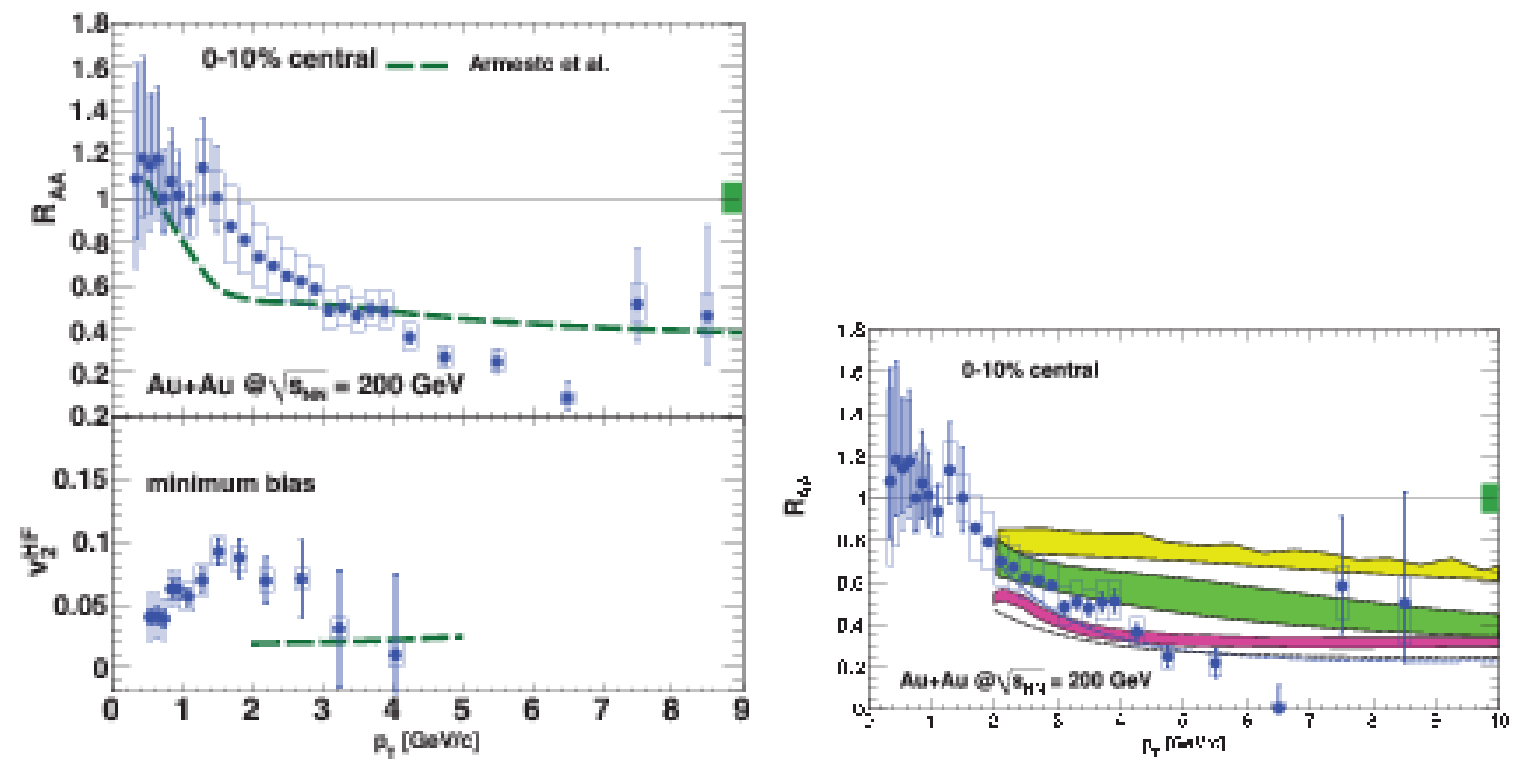

Figure 23: Heavy-flavor electron $R_{\mathrm{AA}}$ in central $\mathrm{Au}+\mathrm{Au}$ collisions and $v_{2}$ in minimum bias collisions predicted from a radiative energy loss calculation within the BDMPS framework [190] (left panel). $\quad R_{\mathrm{AA}}$ in central $\mathrm{Au}+\mathrm{Au}$ collisions from a DGLV calculation 94 with radiative energy loss only (uppermost band) and with additional collisional energy loss (middle band) for electrons from charm and bottom hadron decays. The thin dashed curves correspond to charm hadron decays only. The lowest band shows the prediction from a collisional dissociation model [102 (right panel) (reprinted with permission from Ref. [155]; Copyright (2011) by the American Physical Society).

of electrons from heavy-flavor hadron decays at high $p_{\mathrm{t}}$ and the simultaneous participation of these hadrons in the collective dynamical expansion of the fireball, as indicated by the nonzero flow of decay electrons, can be interpreted in terms of strong interactions of heavy flavor with the hot and dense medium produced at RHIC. By confronting predictions from various theoretical model calculations with the electron data one might hope to unravel the nature of the mechanisms responsible for the observed heavy-flavor medium modifications and, at the same time, approach a quantitative understanding of the properties of hot QCD matter.

A first question is whether the measured $R_{\mathrm{AA}}$ and $v_{2}$ of electrons from heavy-flavor hadron decays require heavy-flavor interactions with the medium on a partonic level or whether (pre)hadronic interactions are sufficient to explain the observed medium effects. The $R_{\mathrm{AA}}$ and $v_{2}$ of $\mathrm{D}$ mesons was calculated in the framework of the hadron-string-dynamics transport model (HSD) [194] which implements heavy-flavor interactions with the medium on a (pre) hadronic level. Only at low $p_{\mathrm{t}}$ the predicted D-meson $R_{\mathrm{AA}}$ is consistent with the electron measurement. Towards higher $p_{\mathrm{t}}$ the calculated D-meson energy loss is not sufficient to account for the small electron $R_{\mathrm{AA}}$. In addition, the substantial $v_{2}$ of electrons from heavy-flavor hadron decays can not be reproduced in this model, indicating that strong partonic interactions are relevant for the observed medium effect on heavy-flavor observables in $\mathrm{Au}+\mathrm{Au}$ collisions at RHIC.

Originally it was expected that the dominant energy loss mechanism of heavy quarks in hot QCD matter at RHIC would be induced gluon radiation, leading to a reduced suppression of heavy quarks with respect to light quarks due to the dead-cone effect. Predictions using the 
BDMPS framework [190], in which radiative partonic energy loss is implemented via multiple soft interactions of the partons with the medium, are confronted with the PHENIX electron data [155] in the left panel of Fig. 23. The relevant parameter concerning the strength of the parton-medium interaction in this model is the transport coefficient $\hat{q}$. For the curves shown in the left panel of Fig. $23 \hat{q}=14 \mathrm{GeV}^{2} / \mathrm{fm}$ was used which is close to the upper limit still consistent with the hadron suppression observed in the light-flavor sector. In particular at high $p_{\mathrm{t}}$, the model prediction is difficult to reconcile with the data if bottom hadron decays contribute significantly to the electron spectra in this region. Since this BDMPS calculation does not include any collective effects at low $p_{\mathrm{t}}$ the comparison of the predicted $v_{2}$ with the data is of limited usefulness. It is interesting to note that the predicted $v_{2}>0$ provides a lower limit of $v_{2}$ at high $p_{\mathrm{t}}$ which is due to the energy loss in the medium in combination with the initial geometry of the collision zone.

An alternative radiate energy loss approach is implemented in the DGLV framework 94,189$]$, in which partons interact with the medium via a few hard collisions. The predicted $R_{\mathrm{AA}}$ of electrons from heavy-flavor hadron decays, shown as the uppermost band in the right panel of Fig. 23, is clearly above the measured $R_{\mathrm{AA}}$ at high $p_{\mathrm{t}}$, consistent with the BDMPS prediction. It was pointed out [99] that for heavy quarks radiative and collisional energy loss via elastic scattering are of similar magnitude over a broad kinematic range at RHIC. Adding collisional energy loss to the DGLV calculation leads to a reduction of the electron $R_{\mathrm{AA}}$ as demonstrated by the middle band in the right panel of Fig. 23 which is still above the measured electron $R_{\mathrm{AA}}$ at high $p_{\mathrm{t}}$.

Following a different Ansatz [195 197], collisional energy loss in pQCD was implemented using a running coupling constant $\alpha_{\text {eff }}$ and replacing the Debye mass $m_{\mathrm{D}}$ by a hard thermal loop calculation. An important parameter in this model is a $K$ factor which is a scaling parameter for the collisional energy loss. $R_{\mathrm{AA}}$ and $v_{2}$ predicted from this model are compared with the data in Fig. 24. With a $K$ factor of 1.8 , the model prediction is in good agreement with the measured $R_{\mathrm{AA}}$ of electrons from heavy-flavor hadron decays, not only for the most central collisions but for all $\mathrm{Au}+\mathrm{Au}$ collision centralities. Furthermore, $K=1.8$ leaves room for additional contributions from radiative or other energy loss mechanisms which are not implemented explicitly. For the calculation of the elliptic flow magnitude $v_{2}$ the interaction time is important in this model, where a later decoupling of heavy quarks from the medium in general can produce larger values of $v_{2}$. The curve shown in the right panel of Fig. 24 corresponds to the situation in which the hadronization of heavy quarks takes place at the end of the mixed phase.

Another class of models uses a Langevin-based transport approach to describe the propagation of heavy quarks in the hot medium, i.e. these quarks are placed into a thermal medium and the quark-medium interaction is implemented by uncorrelated elastic momentum kicks. A relevant parameter in this approach is the heavy-quark diffusion coefficient. The predictions from one of these models [100] are compared with the data for two different values of the diffusion coefficient in the left panel of Fig. 25. While the general features of the data are reproduced by this model, it can not simultaneously describe the measured $R_{\mathrm{AA}}$ and $v_{2}$ of single electrons from heavy-flavor hadron decays with a single value of the heavy-quark diffusion coefficient. In a similar approach, also based on Langevin transport, the elastic scattering of heavy quarks in the medium is mediated by the resonant excitation of charm and bottom states in the medium [101, 198]. Predictions from this model are confronted with the data for two different choices of the resonance widths in the right panel of Fig. 25. Both the measured suppression and the azimuthal anisotropy of electrons from heavy-flavor hadron decays are reproduced by the model calculation. 

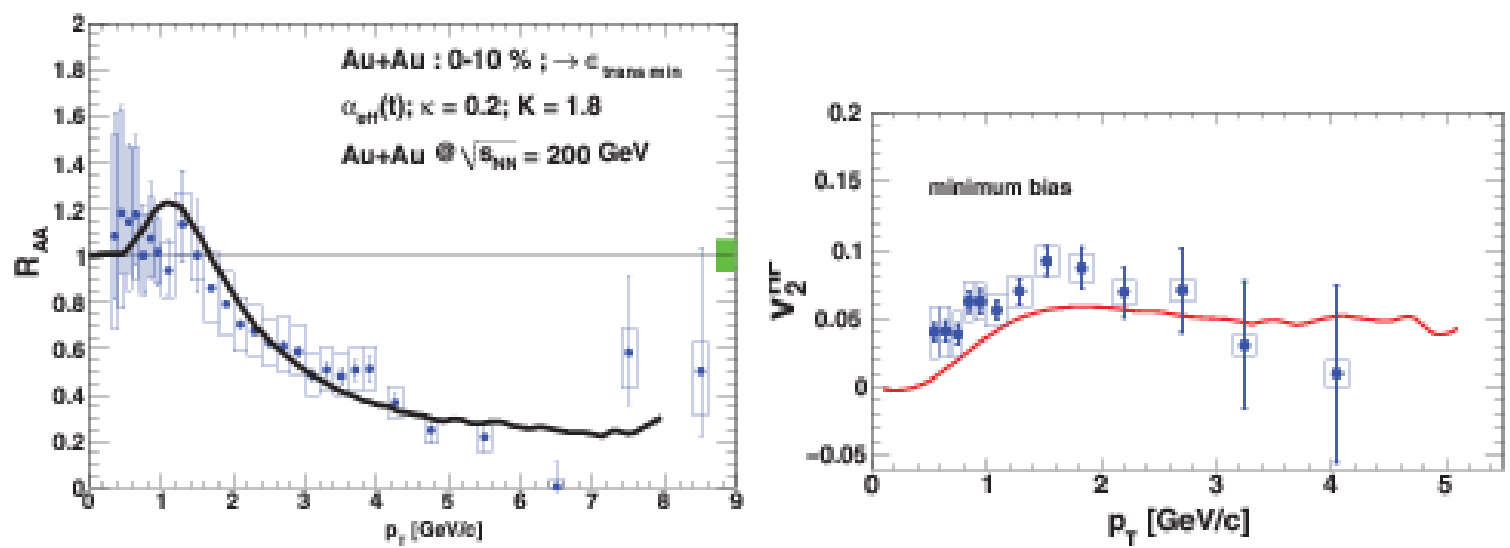

Figure 24: Nuclear modification factor $R_{\mathrm{AA}}$ of electrons from heavy-flavor hadron decays in central $\mathrm{Au}+\mathrm{Au}$ collisions (left panel) and corresponding elliptic flow magnitude $v_{2}$ in minimum bias collisions (right panel) as predicted from a collisional energy loss model [195 197] in comparison with the PHENIX data (reprinted with permission from Ref. [155]; Copyright (2011) by the American Physical Society).

The diffusion constant, which is the driving parameter of both Langevin-based transport models introduced above, can be related to the shear viscosity $\eta$ of the hot QCD matter through which the heavy quarks propagate, where the ratio of $\eta$ to the entropy density $s$ is the quantity that determines the damping rate in the system. In that sense the energy loss of heavy quark in the medium and the simultaneous participation of the heavy quarks in the medium's collective expansion can be interpreted as a damping of the initial heavyquark dynamics by the hot and dense medium. Arguments based on the uncertainty principle exclude zero viscosity for any thermal system [199]. Furthermore, it was shown [43 that specific classes of conformal field theories with gravity duals in the anti-de-Sitter space (AdS/CFT correspondence) exhibit a ratio $\eta / s=1 / 4 \pi$. It was conjectured that this value represents a bound for all relativistic thermal field theories [43]. As it is discussed in detail in Ref. [155, 162], the $R_{\mathrm{AA}}$ and $v_{2}$ measurements of electrons from heavy-flavor hadron decays suggest a value of $\eta / s(1.33-2) / 4 \pi$, close to the conjectured lower bound. In turn, AdS/CFT-based energy loss models [104] give a reasonable description of the measured $R_{\mathrm{AA}}$ of electrons from heavy-flavor hadron decays while the measured $v_{2}$ is severely underestimated. $\eta / s$ can not only be inferred from measurements sensitive to the coupling of heavy quarks to the medium as discussed here, but also from observables related to the bulk of the medium, involving light flavor quarks only. Such estimates based on elliptic flow measurements of light hadrons 200,201 and measurements of fluctuations [202] and entropy production [203] are supported by detailed viscous relativistic hydrodynamic calculations 204,205 and they are consistent with the $\eta / s$ value estimated here using electrons from heavy-flavor hadron decays.

It is an interesting question whether heavy-quark bound states can be formed in hot QCD matter. The relevant pQCD dynamics of open heavy-flavor production was worked out in Ref. [102], and the GLV framework was extended to include composite $q \bar{q}$ systems such that the medium induced dissociation probability of $\mathrm{D}$ and $\mathrm{B}$ mesons propagating through the hot and dense medium could be calculated. In this scenario the sequential fragmentation of heavy quarks and dissociation of heavy-flavor mesons results in an effective energy loss. Quantitatively, the formation time and the detailed expansion dynamics of the produced hot QCD matter as 

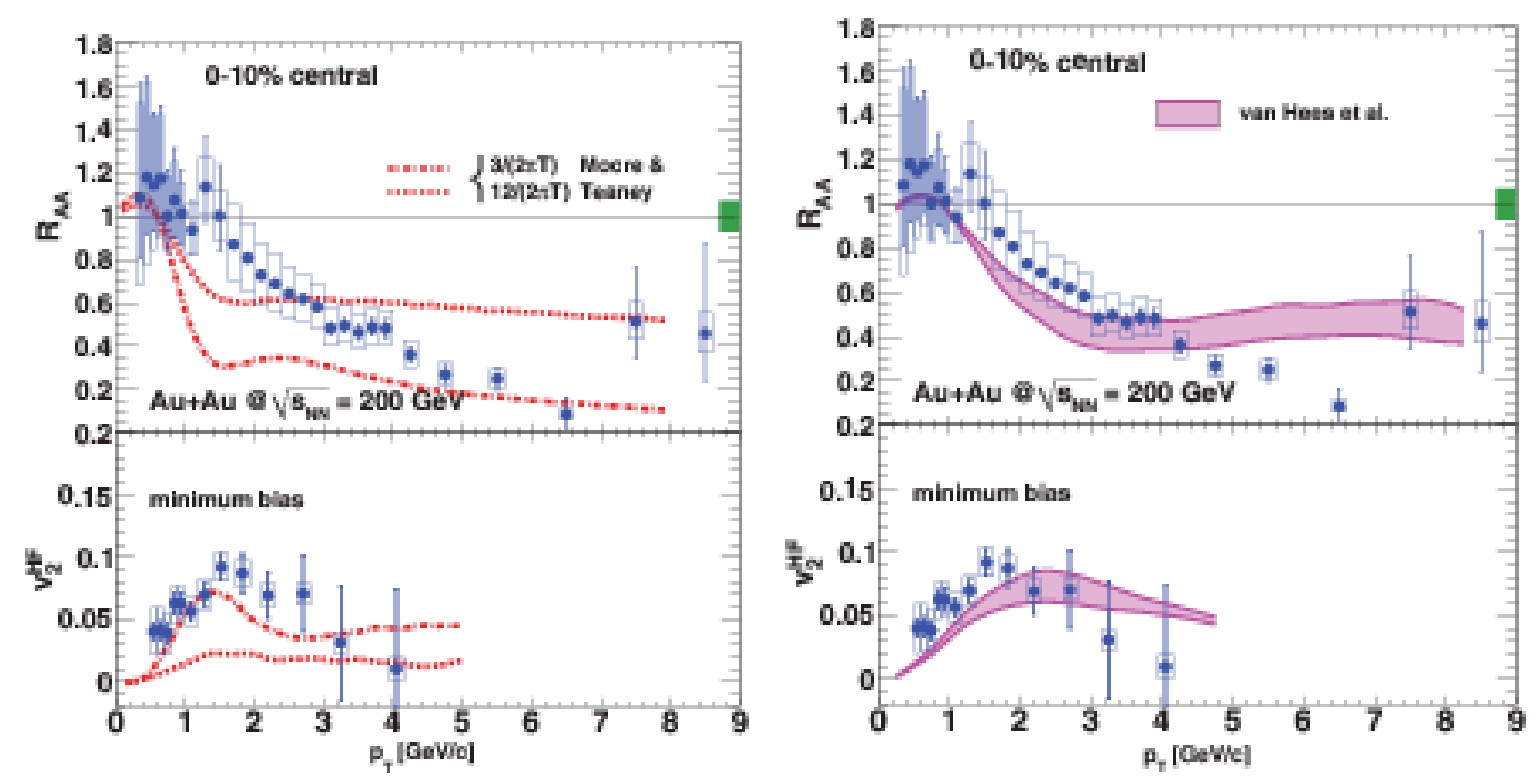

Figure 25: Nuclear modification factor $R_{\mathrm{AA}}$ of electrons from heavy-flavor hadron decays in central $\mathrm{Au}+\mathrm{Au}$ collisions and corresponding elliptic flow magnitude $v_{2}$ in minimum bias collisions as predicted from Langevin-based transport models without 100 (left panel) and with 101,198 (right panel) resonance excitation of heavy-flavor like hadrons in the medium. See text for details (reprinted with permission from Ref. [155]; Copyright (2011) by the American Physical Society).

well as the in-medium formation time of the heavy-flavor hadrons play a role. The resulting nuclear modification factor of electrons from heavy-flavor hadron decays, shown as the lowest band in the right panel of Fig. 23, is in reasonable agreement with the measurement as are result from improved calculations which combine sequential heavy-flavor hadron formation and dissociation with charm and bottom energy loss on the parton level [103].

In all above model calculations, addressing the production of heavy-flavor hadron decay electrons, the same chemical composition of charm and bottom hadrons is assumed for both pp and $\mathrm{Au}+\mathrm{Au}$ collisions. However, in the light-flavor sector an enhancement of baryon yields at intermediate $p_{\mathrm{t}}$ relative to meson yields has been observed in central $\mathrm{Au}+\mathrm{Au}$ collisions at RHIC [107]. A similar baryon anomaly in the heavy-quark sector could lead to an enhancement of $\Lambda_{\mathrm{c}}$ relative to $\mathrm{D}$ mesons. Given the smaller semileptonic decay branching ratio of charm baryons compared to mesons and because of a softer $p_{\mathrm{t}}$ spectrum of electrons from charm baryon decays, $R_{\mathrm{AA}}<1$ would be a consequence of such a baryon anomaly 108 even without any additional heavy-flavor energy loss in the hot QCD matter. Assuming a charm baryon to meson ratio close to one a nuclear modification factor of about 0.65 was predicted [109 for electrons from heavy-flavor hadron decays. Without additional experimental input it will be impossible to resolve this issue. Clearly, the reconstruction of displaced secondary decay vertices and exclusive measurements of hadronic heavy-flavor decays are highly desirable as they would not suffer from such ambiguities.

The issue of charm versus bottom energy loss suffers from similar ambiguities. The relative contribution, $r_{\mathrm{B}}$, from bottom hadron decays to the electron spectra from heavy-flavor hadron decays has been measured with substantial uncertainties in pp collisions [163, 175]. For $\mathrm{Au}+\mathrm{Au}$ 


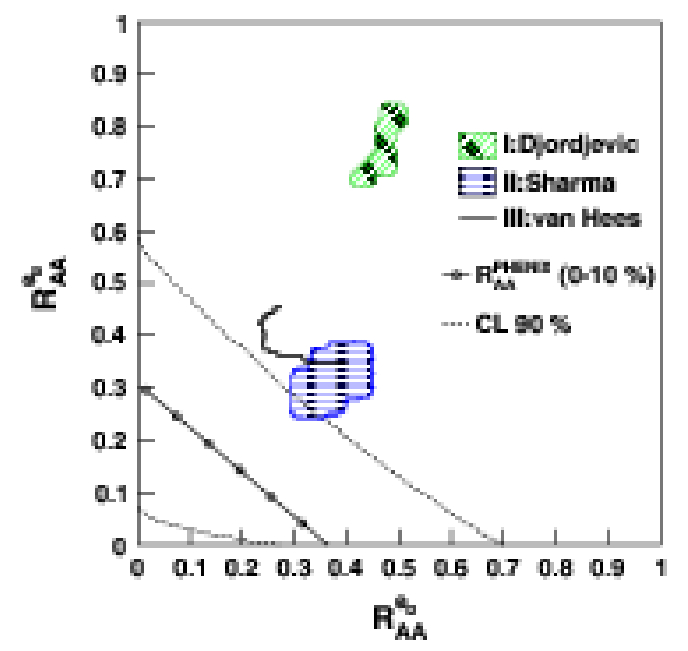

Figure 26: $90 \%$ confidence level contours for $R_{\mathrm{AA}}$ of electrons from D-meson and B-meson decays for electron $p_{\mathrm{t}}>5 \mathrm{GeV} / c$ in comparison with model calculations. See text for more details (reprinted with permission from Ref. [175]; Copyright (2010) by the American Physical Society).

collisions, however, experimental constraints are missing. Therefore, the only practical approach to separate the nuclear modification factors of electrons from charm hadron decays, $R_{\mathrm{AA}}^{e_{\mathrm{D}}}$, and from bottom hadron decays, $R_{\mathrm{AA}}^{e_{\mathrm{B}}}$, is to combine the measurement of $r_{\mathrm{B}}$ in pp collisions with the measurement of $R_{\mathrm{AA}}^{e_{\mathrm{AF}}}$ of electrons from all heavy-flavor hadron decays in $\mathrm{Au}+\mathrm{Au}$ collisions via the equation

$$
R_{\mathrm{AA}}^{e_{\mathrm{HF}}}=\left(1-r_{\mathrm{B}}\right) R_{\mathrm{AA}}^{e_{\mathrm{D}}}+r_{\mathrm{B}} R_{\mathrm{AA}}^{e_{\mathrm{B}}} .
$$

Since above an electron $p_{\mathrm{t}}$ of about $5 \mathrm{GeV} / c$ both $r_{\mathrm{B}}$, as measured with the STAR experiment in pp collisions [175], and $R_{\mathrm{AA}}^{e_{\mathrm{HF}}}$, as measured in central $\mathrm{Au}+\mathrm{Au}$ collisions with the PHENIX experiment 155, 162], do not show a significant $p_{\mathrm{t}}$ dependence, such an attempt to separate the contributions from charm and bottom hadrons decays was conducted in this $p_{\mathrm{t}}$ region as discussed in detail in Ref. [175]. The results are summarized in Fig. 26, which shows the most probable values for $R_{\mathrm{AA}}^{e_{\mathrm{D}}}$ and $R_{\mathrm{AA}}^{e_{\mathrm{B}}}$ obtained from the data as open circles and the corresponding $90 \%$ confidence limits as dashed lines. Clearly the data imply a substantial suppression not only of electrons from charm but also from bottom hadron decays. The predictions from three models are confronted with the data in Fig. 26. A DGLV calculation with radiative energy loss only (model I) [189] predicts a too small suppression for charm and, in particular, bottom energy loss. A collisional energy loss model including collisional dissociation, partonic energy loss, and cold nuclear matter effects (model II) [103] is in reasonable agreement with the data as is a model in which collisional energy loss is mediated by resonant elastic scattering (model III) [101. Not shown are calculations within the AdS/CFT framework [206, 207], which also predict small $R_{\mathrm{AA}}$ for electrons from charm and bottom hadron decays. Even more important, the ratio of the nuclear modification factors of charm and bottom are predicted [208] to be significantly different for AdS/CFT calculations and pQCD-based calculations as shown for LHC energies in Fig. 27, emphasizing the importance of separate measurements of charm and bottom hadron energy loss in the future. 


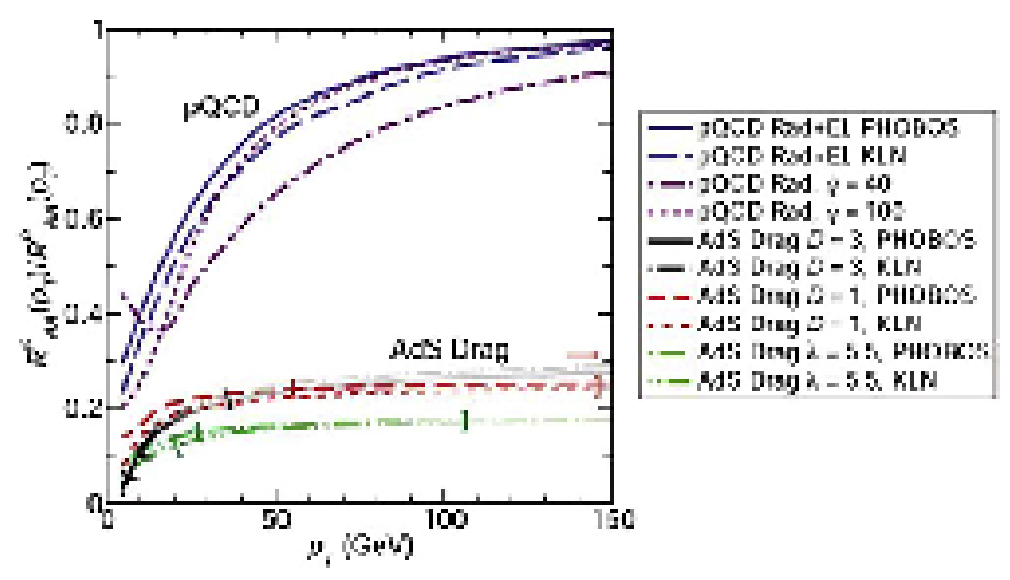

Figure 27: Ratio of the nuclear modification factors of charm and bottom calculated within the frameworks of AdS/CFT [208] and WHDG pQCD [94] with a wide range of input parameters as a function of $p_{\mathrm{t}}$ (reprinted from Ref. 208]).

In summary, the measurements of electrons from heavy-flavor hadron decays in pp and $\mathrm{Au}+\mathrm{Au}$ collisions at RHIC as reviewed here constitute the first systematic application of open heavy-flavor observables as a tool to probe the properties of the hot QCD matter produced in high-energy nucleus-nucleus collisions. It was shown that the total charm yield scales proportional to the number of binary collisions in nucleus-nucleus collisions at RHIC, i.e. charm quark-antiquark pairs are almost exclusively produced in point-like hard scattering processes. Additional production processes such as thermal production in the hot medium do not play a significant role. The produced heavy quarks propagate through the medium where they are subject to strong interactions with the medium, which do not change the overall heavy-flavor yields but modify the phase-space distributions to a large extent. Electrons from heavy-flavor hadron decays are suppressed at high $p_{\mathrm{t}}$, quantitatively similar to what is observed for light hadrons. This observation is in stark contrast with the expectation from energy-loss models based on radiative energy-loss mechanisms. Collisional energy loss can not be ignored for heavy quarks and/or alternative energy-loss mechanism need to be considered. At the same time, the measured $v_{2}$ of these electrons indicates that charm quarks participate in the collective expansion of the produced hot QCD medium. These observations support the characterization of the hot and dense QCD matter formed in $\mathrm{Au}+\mathrm{Au}$ collisions at RHIC as a strongly interacting medium with partonic degrees of freedom which features a minimal ratio of shear viscosity to entropy density and, therefore, was dubbed an almost perfect fluid. However, semielectronic heavy-flavor hadron decays provide only indirect information. In particular, a precision separation of charm from bottom is difficult via this approach, and the chemistry of charm and bottom hadron states is completely inaccessible. These issue can only be addressed with the upgrades of the PHENIX and STAR experiments.

\subsubsection{Semimuonic heavy-flavor hadron decays}

While extensive systematic studies of heavy-flavor production in heavy-ion collisions have been conducted at RHIC in the semielectronic decay channel at mid-rapidity, corresponding measurements in the semimuonic decay channel at forward rapidity have become available only 

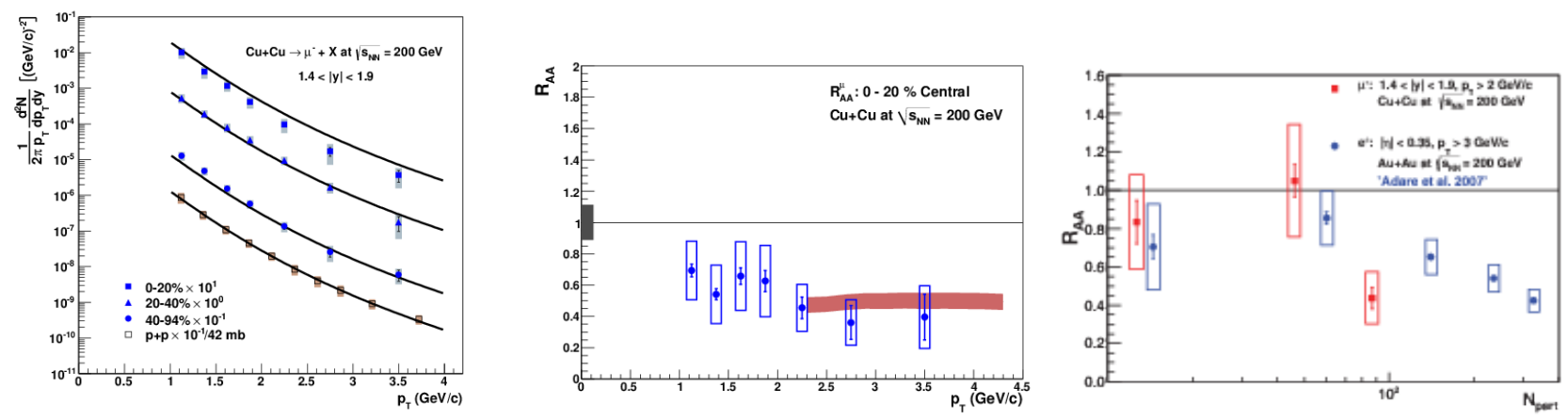

Figure 28: $p_{\mathrm{t}}$-differential invariant yields of negatively charged muons from heavy-flavor hadron decays in $\mathrm{pp}$ and three centrality classes of $\mathrm{Cu}+\mathrm{Cu}$ collisions measured at forward rapidity with the PHENIX experiment at $\sqrt{s_{\mathrm{NN}}}=200 \mathrm{GeV}$. The solid lines depict a fit to the pp data scaled by the appropriate number of binary collisions to the $\mathrm{Cu}+\mathrm{Cu}$ centrality classes (left panel). Nuclear modification factor $R_{\mathrm{AA}}$ for negative muons from heavy-flavor hadron decays measured as a function of $p_{\mathrm{t}}$ in the $20 \%$ most central $\mathrm{Cu}+\mathrm{Cu}$ collisions. For comparison, a prediction from a collisional dissociation model [103] is shown (middle panel). Comparison of the nuclear modification factors of heavy-flavor muons at forward rapidity $(1.4<y<1.9)$ and $p_{\mathrm{t}}>2 \mathrm{GeV} / c$ in $\mathrm{Cu}+\mathrm{Cu}$ collisions $[156$ and heavy-flavor electrons at mid-rapidity $(|\eta|<0.35)$ and $p_{\mathrm{t}}>3 \mathrm{GeV} / c$ in $\mathrm{Au}+\mathrm{Au}$ collisions 162 as a function of the collision centrality, expressed by the number of participating nucleons (right panel) (reprinted with permission from Ref. [156]; Copyright (2012) by the American Physical Society).

recently.

With the PHENIX experiment negatively charged muons from the decay of heavy-flavor hadrons have been measured in the rapidity range $1.4<y<1.9$ and for transverse momenta $1<p_{\mathrm{t}}<4 \mathrm{GeV} / c$ in three centrality classes of $\mathrm{Cu}+\mathrm{Cu}$ collisions at $\sqrt{s_{\mathrm{NN}}}=200 \mathrm{GeV}$ [156]. Background, mainly from light hadrons, was estimated using a MonteCarlo approach and it was subtracted statistically from the inclusive muon candidate sample. The resulting invariant yields measured in $\mathrm{Cu}+\mathrm{Cu}$ collisions are compared as a function of the muon $p_{\mathrm{t}}$ with the corresponding data from pp collisions in the left panel of Fig. 28, where the solid lines correspond to a fit of the pp data scaled with the appropriate numbers of binary collisions to the $\mathrm{Cu}+\mathrm{Cu}$ centrality classes. While the muon yields from heavy-flavor hadron decays in peripheral and mid-central $\mathrm{Cu}+\mathrm{Cu}$ collisions are in reasonable agreement with the binary collision scaled pp reference, for the $20 \%$ most central $\mathrm{Cu}+\mathrm{Cu}$ collisions a suppression of such muons is observed at high $p_{\mathrm{t}}$ relative to the pp fit.

This suppression is quantified via the nuclear modification factor $R_{\mathrm{AA}}$ of muons from heavyflavor hadron decays shown as a function of $p_{\mathrm{t}}$ in the middle panel of Fig. 28 for the $20 \%$ most central $\mathrm{Cu}+\mathrm{Cu}$ collisions. A prediction from a theoretical model [103] agrees well with the measurement at high $p_{\mathrm{t}}$. This specific model includes elastic and inelastic in-medium energy loss of heavy quarks, the dissociation of heavy mesons in medium, and cold nuclear matter effects such as shadowing and initial state energy loss due to multiple scattering of the incoming partons [103]. A related model prediction [102] is also in reasonable agreement with the measured nuclear modification factor of electrons from heavy-flavor hadron decays in $\mathrm{Au}+\mathrm{Au}$ collisions at mid-rapidity (see Fig. 23). 
As demonstrated in the right panel of Fig. 28, the suppression of muons from heavy-flavor hadron decays at forward rapidity in central $\mathrm{Cu}+\mathrm{Cu}$ collisions is comparable to the suppression observed in central Au+Au collisions for electrons from heavy-flavor hadron decays [155, 162. This is a surprising observation since the Bjorken energy density of hot QCD matter produced at mid-rapidity in central $\mathrm{Au}+\mathrm{Au}$ collisions at $\mathrm{RHIC}$ is expected to be larger by at least a factor of two compared to the energy density in the forward rapidity region in $\mathrm{Cu}+\mathrm{Cu}$ collisions. Consequently, the substantial suppression observed in $\mathrm{Cu}+\mathrm{Cu}$ collisions might point to significant cold nuclear matter effects playing a role in particular at forward rapidity.

Major progress at forward rapidity requires improved heavy-flavor production measurements at RHIC. In particular, the PHENIX forward silicon vertex detector will allow the separation of charm and bottom contributions to the muon sample from heavy-flavor hadron decays.

\section{Open heavy-flavor measurements at the LHC}

\subsection{Experiments studying heavy-flavor production at the LHC}

The LHC delivered the first pp collisions at injection energy, i.e. at $\sqrt{s}=0.9 \mathrm{TeV}$ in November 2009. While data were recorded with all experiments during the commissioning phase at this energy, the accumulated statistics was not sufficient to investigate the production of heavyflavor hadrons. Substantial data sets, providing already detailed information on heavy-flavor production in pp collisions, were recorded by all four large LHC experiments at an energy of $\sqrt{s}=7 \mathrm{TeV}$ in the years 2010/2011. In particular, these data provided a reference for the first $\mathrm{Pb}+\mathrm{Pb}$ collision data recorded at the LHC at $\sqrt{s_{\mathrm{NN}}}=2.76 \mathrm{TeV}$ in November/December 2010 and 2011 with the ALICE, ATLAS, and CMS experiments. The LHCb experiment does not participate in heavy-ion runs. Because of the difference in the available energy per nucleonnucleon pair the $7 \mathrm{TeV}$ pp data have to be scaled to the lower energy of $2.76 \mathrm{TeV}$ to provide a reference for measurements in $\mathrm{Pb}+\mathrm{Pb}$ collisions. As will be demonstrated in Section 4.2 , all heavy-flavor production data from $7 \mathrm{TeV}$ pp collisions at the LHC are in good agreement with predictions from FONLL pQCD calculations [83]. Therefore, FONLL driven $\sqrt{s}$ scaling procedures could be used to determine pp references at the proper energy for $\mathrm{Pb}+\mathrm{Pb}$ collisions. In order to validate the FONLL based $\sqrt{s}$ scaling and to provide experimental references for observables which can not be scaled by pQCD model calculations, the LHC delivered pp collisions at the energy of $\sqrt{s}=2.76 \mathrm{TeV}$ for a short period of a few days in March 2011. While the LHC operates at a collision energy of $\sqrt{s}=8 \mathrm{TeV}$ since April 2012 relevant results from this experimental pp campaign are not yet available.

This review discusses two issues related to the LHC experiments. First, it is demonstrated that perturbative QCD calculations are able to reasonably well describe heavy-flavor hadron production in pp collisions at the LHC, using data from all four large experiments in the hadronic and semileptonic decay channels at mid-rapidity and at forward rapidity. It is actually a big success of the experiments as well as of the theoretical calculations that this can be concluded already during the very first years of LHC operations. It took years to resolve discrepancies that seemingly existed between pQCD calculations and measurements at the Fermilab Tevatron. Also at RHIC, long standing discrepancies between the electron spectra from heavy-flavor hadron decays measured with the PHENIX and STAR experiments, respectively, could be resolved only after a while. Second, the first published results on open heavy-flavor production in $\mathrm{Pb}+\mathrm{Pb}$ collisions obtained with the ALICE and CMS experiments are summa- 
rized. While these measurements confirm the substantial suppression of heavy-flavor probes at high $p_{\mathrm{t}}$ with respect to binary collision scaled pp measurements as observed at RHIC, they also represent a major step forward not only in terms of the energy scale. For the first time, hot QCD matter effects on exclusive heavy-flavor hadron observables can be investigated. In $\mathrm{Pb}+\mathrm{Pb}$ collisions, $\mathrm{D}$ mesons have been reconstructed with the ALICE experiment and with the CMS experiment $\mathrm{J} / \psi$ mesons originating from b-hadron decays have been studied. Such measurements have only been possible because all of the four large LHC experiments include precision silicon vertex detectors, which allow the experimental separation of displaced secondary vertices from heavy-flavor hadron decays from the primary collision vertex. Augmented by inclusive measurements, e.g. of electrons and muons from semileptonic heavy-flavor hadron decays, these first exclusive results are only the tip of the iceberg of near time future results to be expected from systematic heavy-flavor production studies with all experiments at the LHC. In the following, the experimental approaches of the four large LHC experiments to open heavy-flavor measurements are summarized briefly, with special emphasis on the ALICE experiment.

\subsubsection{The ALICE approach to open heavy-flavor measurements}

The ALICE experiment [209] was designed and optimized to cope with the large particle multiplicities in central $\mathrm{Pb}+\overline{\mathrm{Pb}}$ collisions at the LHC. The ALICE apparatus consists of two main parts: a central barrel detector system housed inside the L3 solenoid magnet, which provides a field of $0.5 \mathrm{~T}$, and a forward muon spectrometer $(-4<\eta<-2.5)$ including a dipole magnet, which provides a field integral of $3 \mathrm{Tm}$. This setup is augmented by several smaller auxiliary detectors for event characterization and for triggering purposes. Special emphasis was put on excellent tracking and particle identification capabilities, in particular also at low momenta. With these capabilities excellent measurements of heavy-flavor production are possible through the reconstruction of heavy-flavor hadrons in their hadronic decay channels at mid-rapidity as well as via semileptonic decays of charm and bottom hadrons both at mid-rapidity and forward rapidity.

The central barrel tracking system consists of the silicon Inner Tracking System (ITS) and the Time Projection Chamber (TPC). In the pseudorapidity range $|\eta|<0.9$ tracks are reconstructed with a momentum resolution better than $4 \%$ for $p_{\mathrm{t}}<20 \mathrm{GeV} / c$. The distance of closest approach of tracks to the interaction vertex is measured with a resolution better than $75 \mu \mathrm{m}$ for $p_{\mathrm{t}}>1 \mathrm{GeV} / c$ in the plane transverse to the beam direction [210].

Charged hadrons are identified via their specific energy loss $\mathrm{d} E / \mathrm{d} x$ in the TPC and a timeof-flight (TOF) measurement in the ALICE TOF detector. The same techniques allow a clean electron identification in the range $p_{\mathrm{t}}<6 \mathrm{GeV} / c$. At higher $p_{\mathrm{t}}$, the Transition Radiation Detector (TRD) and, alternatively, the Electromagnetic Calorimeter (EMCal) are necessary for the measurement of a pure electron sample.

The measurement of $\mathrm{D}$ mesons at mid-rapidity $(|y|<0.5)$ is based on the selection of displaced-vertex topologies [210]. Tracks are selected which originate from a secondary vertex consistent with a large decay length as expected from the D-meson lifetimes. In addition, a good alignment of the reconstructed D-meson momentum with the flight line from the collision vertex to the secondary vertex is required. Since the relevant D-meson decay channels $\left(\mathrm{D}^{0} \rightarrow \mathrm{K}^{-} \pi^{+}\right.$, $\mathrm{D}^{+} \rightarrow \mathrm{K}^{-} \pi^{+} \pi^{+}$, and $\mathrm{D}^{*+} \rightarrow \mathrm{D}^{0} \pi^{+}$) involve a kaon as decay product the identification of the charged kaon in the TPC and TOF detectors helps to reduce the background. In an invariant mass analysis the raw D-meson yields are determined which, then, are corrected for geometrical 
acceptance and for reconstruction and particle identification efficiency based on a detailed simulation of the apparatus. Feed down from B-meson decays is estimated from a FONLL pQCD calculation 83 and it is subtracted.

Bottom production can be investigated via the measurement of $\mathrm{J} / \psi$ mesons from the decay of bottom hadrons, $c f$.Tab. 1. $\mathrm{J} / \psi$ mesons are reconstructed via their $e^{+} e^{-}$decays at mid-rapidity $(|y|<0.9)$. The fraction of the total $\mathrm{J} / \psi$ yield originating not from the primary collision vertex but from a displaced bottom-hadron decay vertex is determined via the measurement of the pseudo-proper decay length of the $\mathrm{J} / \psi$ mesons [211].

Electrons from heavy-flavor hadron decays are measured at mid-rapidity [212] in a two step procedure. First, the inclusive electron spectrum is determined. Electron candidate tracks are identified with the TPC, TOF, TRD, and EMCal detectors. The small remaining hadron background is estimated via fits of the TPC $\mathrm{d} E / \mathrm{d} x$ distribution in momentum slices and it is subtracted from the electron candidate spectra. After corrections for geometrical acceptance and tracking and particle identification efficiencies derived from simulations the inclusive electron spectrum is obtained. From this inclusive spectrum a cocktail of electrons from sources other than heavy-flavor decays is subtracted. Similar to corresponding analyses at RHIC, the most important components of this background cocktail are Dalitz decays of the $\pi^{0}$ and $\eta$ meson as well as photon conversions in the beam pipe and in the first pixel layer of the ITS. In addition, decays of light vector mesons $(\rho, \omega, \phi)$, heavy quarkonia $(J / \psi, \Upsilon)$, and direct radiation from hard scattering processes are included. The cocktail input is based on the $\pi^{0}$ and $\eta$ spectra measured with the ALICE experiment [213], the known material budget of the apparatus [214], $m_{\mathrm{t}}$ scaling for the $p_{\mathrm{t}}$ spectra and yield of light vector mesons, heavy quarkonia measurements with the ALICE [215] and CMS experiments [216, 217], and next-to-leadingorder pQCD calculations 218, 219 for the electron spectra from direct radiation. With the ALICE ITS, electrons originating from displaced secondary bottom-hadron decay vertices can be separated from decay vertices of shorter lived charm hadrons and from the primary collision vertex. This technique allows the measurement of electrons from bottom-hadron decays [220].

Muons with momenta above $4 \mathrm{GeV} / c$ are identified and their momenta are measured in the pseudorapidity range $-4<\eta<-2.5$ with the ALICE forward muon spectrometer, which consists of a 10 interaction lengths passive front absorber, a small angle beam shield, a set of tracking chambers in front of, in, and behind a $3 \mathrm{Tm}$ dipole magnet, and trigger chambers located behind an iron wall with a thickness of 7.2 interaction lengths. From the inclusive muon candidate spectrum three main background sources have to be removed: muons from the decay of primary light hadrons, muons from the decay of hadrons produced in secondary interactions in the front absorber, and hadrons that punch through the front absorber. Contributions from the decay of $\mathrm{W}^{ \pm}$and $Z^{0}$ vector bosons are negligible in the muon $p_{\mathrm{t}}$ range $2<p_{\mathrm{t}}<12 \mathrm{GeV} / c$. Punch through hadrons are rejected efficiently requiring that muon candidate tracks match a track in the muon trigger system. Decay muons are the dominant source of background in the relevant $p_{\mathrm{t}}$ region. In pp collisions 221] this contribution can be subtracted using information from detailed simulations. In $\mathrm{Pb}+\mathrm{Pb}$ collisions 222 the presence of unknown medium effects prevents such a MonteCarlo approach. Instead, the decay muon contribution is estimated by extrapolating the $p_{\mathrm{t}}$ spectra of pions and kaons measured in $\mathrm{Pb}+\mathrm{Pb}$ collisions at mid-rapidity [223] to forward rapidity and calculating the corresponding decay muon spectra. 


\subsubsection{The ATLAS approach to open heavy-flavor measurements}

The ATLAS collaboration operates one of the two large general purpose detectors at the LHC designed to extend the frontiers of particle physics by exploiting the unprecedented high collision energy and luminosity delivered by the LHC. The main goals of ATLAS are related to the search for the Standard Model Higgs boson, precision tests of QCD, electroweak interactions, flavor physics, and the search for physics beyond the Standard Model in pp collisions. Utilizing mainly hard probes such as jets, ATLAS also studies the $\mathrm{Pb}+\mathrm{Pb}$ collisions provided by the LHC. In the following, a brief characterization of those subsystems of the ATLAS apparatus is given which are relevant for the results published on open heavy-flavor production in pp collisions up to now. ATLAS has not yet published open heavy-flavor data from $\mathrm{Pb}+\mathrm{Pb}$ collisions.

The ATLAS apparatus covers almost the full solid angle around the interaction point. An inner detector (ID) tracking system consisting of a 3 layer silicon pixel detector, a 4 layer double-sided silicon strip detector, and a 73 layer transition radiation straw tube tracker is located in a $2 \mathrm{~T}$ solenoidal magnetic field, and it covers the pseudorapidity range $|\eta|<2.5$. The ID is surrounded by a high-granularity liquid-argon electromagnetic calorimeter (EM), also covering $|\eta|<2.5$, and hadron calorimetry provided by an iron-scintillator tile calorimeter in the mid-rapidity region and copper/liquid-argon endcap calorimeters towards forward and backward rapidity. The outer muon spectrometer (MS) is located inside a toroidal magnetic field providing a bending power of $2.5 \mathrm{Tm}$ in the barrel and $5 \mathrm{Tm}$ in the end caps. Muons are tracked in the region $|\eta|<2.7$ using monitored drift tube chambers and higher granularity cathode strip chambers in the end caps. Muon trigger chambers employ resistive plate chambers for $|\eta|<1.05$ and thin gap chambers in the region $1.05<|\eta|<2.4$. This general purpose setup provides ample opportunities for the measurement of open heavy-flavor observables. A detailed descriptions of the detector layout of the ATLAS experiment can be found elsewhere [224].

\subsubsection{The CMS approach to open heavy-flavor measurements}

The CMS collaboration operates the second large general purpose detector at the LHC. The main goals of CMS are identical to the ones of ATLAS (see Sec. 4.1.2). Open heavy-flavor measurement in $\mathrm{pp}$ and $\mathrm{Pb}+\mathrm{Pb}$ collisions with the CMS experiment at the $\mathrm{LHC}$ have used the inner tracker and the muon system of the apparatus up to now. Therefore, these detector components are briefly characterized below. A detailed descriptions of the detector layout can be found elsewhere 225].

The central component of the CMS apparatus is a superconducting solenoid with an internal diameter of $6 \mathrm{~m}$, providing at $3.8 \mathrm{~T}$ magnetic field. Immersed in the magnetic field are the inner tracker surrounded by a lead-tungstate crystal electromagnetic calorimeter and a brass/scintillator hadron calorimeter. The silicon pixel tracker comprises three barrel layers and two endcap disks at each barrel end. This device is surrounded by a silicon strip tracker consisting of 10 barrel layers and 12 endcap disks at each barrel end. Muons are detected in the pseudorapidity interval $|\eta|<2.4$ with three types of gas-ionization detectors embedded in the magnet's steel return yoke: drift tubes, cathode strip chambers, and resistive plate chambers. Due to the strong magnetic field and the silicon tracker's high granularity a $p_{\mathrm{t}}$ resolution of about $1 \%$ is reached for muon candidates which are matched with reconstructed tracks. The silicon tracker also provides the position of the primary collision vertex with an accuracy of about $20 \mu \mathrm{m}$ allowing the separation of primary tracks from those originating from displaced secondary decay vertices. Weak decays of hadrons, e.g. $K_{S}^{0} \rightarrow \pi^{+} \pi^{-}$, can be reconstructed via an invariant mass analysis of charged particle tracks pointing back to a common decay 
vertex and fulfilling decay topology cuts, even without the identification of the decay hadron species. In addition, the CMS experiment includes substantial forward calorimetry in the pseudorapidity range $2.9<\eta<5.2$ as well as auxiliary forward detectors for triggering purposes and beam-halo rejection. In $\mathrm{Pb}+\mathrm{Pb}$ collisions information provided by the forward detectors is used for event selection and the event-by-event centrality determination. As in the case of ATLAS, the CMS experiment gives access to open heavy-flavor observables in a multitude of channels, not all of which have been addressed in publications yet.

\subsubsection{The LHCb approach to open heavy-flavor measurements}

The LHCb experiment was designed as a single-arm forward spectrometer 226 aiming at the measurement of $\mathrm{CP}$ violating and rare decays of heavy-flavor hadrons in pp collisions at the $\mathrm{LHC} . \mathrm{Pb}+\mathrm{Pb}$ collisions are not investigated with the LHCb experiment. Nevertheless, in the context of this review LHCb provides an important testing ground for pQCD calculations regarding the rapidity dependence of heavy-flavor production cross sections in pp collisions at the LHC.

The LHCb apparatus covers the pseudorapidity range $2<\eta<5$. The detector includes a high precision tracking system comprising a silicon strip vertex detector surrounding the nominal pp interaction point, a large-area silicon strip detector located upstream of a dipole magnet providing a bending power of $4 \mathrm{Tm}$, and three stations of silicon strip detectors and straw drift tubes located downstream. With this tracking system a momentum resolution $\Delta p / p$ is achieved between $0.4 \%$ at $5 \mathrm{GeV} / c$ and $0.6 \%$ at $100 \mathrm{GeV} / c$. For high $p_{\mathrm{t}}$ tracks, the impact parameter is measured with a resolution of $20 \mu \mathrm{m}$. Two ring imaging Cherenkov detectors are used for hadron identification. Photon, electron, and hadron candidates are selected with a calorimeter system including scintillating pad and pre shower detectors, an electromagnetic and a hadronic calorimeter. Alternating layers of iron absorbers and multiwire proportional chambers compose the muon detection system.

In addition to a variety of results concerning $\mathrm{CP}$ violating and rare heavy-flavor hadron decays, the LHCb collaboration has also published baseline cross sections of bottom production in pp collisions at $\sqrt{s}=7 \mathrm{TeV}$, which can be compared with predictions from pQCD calculations.

\subsection{Reference measurements in pp collisions}

As for the RHIC case, open heavy-flavor measurements in pp collisions at $\sqrt{s}=7 \mathrm{TeV}$ at the LHC provide a crucial testing ground for higher order pQCD calculations in a yet unexplored high energy regime and, at the same time, they deliver a baseline for corresponding measurements in $\mathrm{Pb}+\mathrm{Pb}$ collisions, where the interaction of heavy-flavor particles with the produced hot and dense QCD medium is expected to leave its footprint on open heavy-flavor observables.

\subsubsection{Hadronic heavy-flavor hadron decays}

The only measurement of mid-rapidity charm hadron production cross sections in pp collisions at the LHC via the full reconstruction of hadronic decay channels has been conducted with the ALICE experiment [210]. $\mathrm{D}^{0}, \mathrm{D}^{+}$, and $\mathrm{D}^{*+} p_{\mathrm{t}}$-differential production cross sections measured in the range $|y|<0.5[210]$ are shown in Fig. 29. Theoretical calculations based on higher-order pQCD calculations (FONLL 83 and GM-VFNS [84]) are in reasonable agreement with the data within substantial experimental and theoretical systematic uncertainties. 

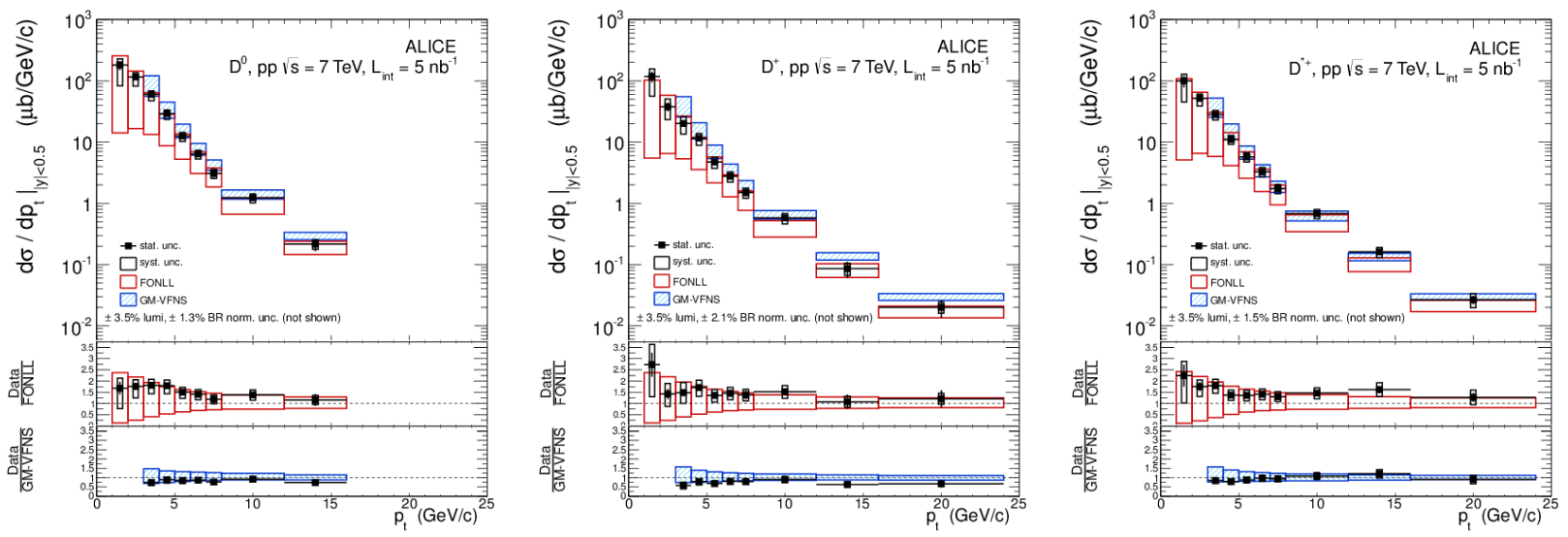

Figure 29: $p_{\mathrm{t}}$-differential production cross sections of $\mathrm{D}^{0}$ (left panel), $\mathrm{D}^{+}$(middle panel), and $\mathrm{D}^{*+}$ (right panel) measured at mid-rapidity $(|y|<0.5)$ with the ALICE experiment in pp collisions at $\sqrt{s}=7 \mathrm{TeV}$ [210] in comparison with pQCD calculations [83, 84]. The lower panels of the individual plots show the ratio of the measured cross sections and the corresponding predictions from higher order FONLL and GM-VFNS pQCD calculations (reprinted from Ref. [210] with kind permission from Springer Science and Business Media).
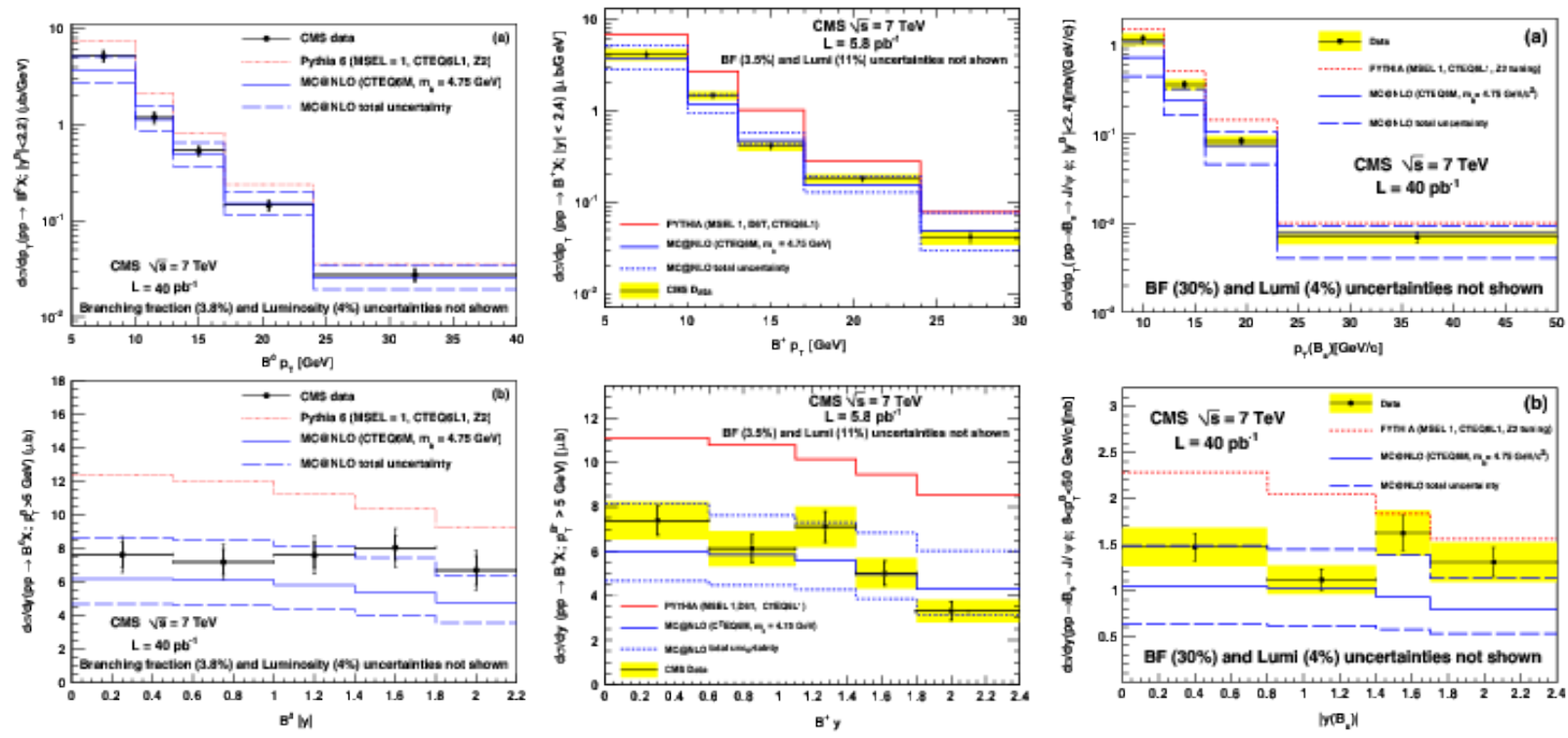

Figure 30: $p_{\mathrm{t}^{-}}$(upper panels) and rapidity-differential (lower panels) production cross sections of $\mathrm{B}^{0}$ (left panels), $\mathrm{B}^{+}$(middle panels), and $\mathrm{B}_{\mathrm{s}}^{0}$ (right panels) mesons measured with the CMS experiment in pp collisions at $\sqrt{s}=7 \mathrm{TeV}$. Predictions from higher order pQCD calculations (MC@NLO [230|) are compared with the data (reprinted with permission from Refs. [227- 229]; Copyright (2011) by the American Physical Society). 
The situation is similar for the production of bottom hadrons in pp collisions at the LHC as demonstrated in Fig. 30 which shows the production cross sections of $\mathrm{B}^{0}, \mathrm{~B}^{+}$, and $\mathrm{B}_{\mathrm{s}}^{0}$ mesons as a function of $p_{\mathrm{t}}$ and, for high $p_{\mathrm{t}}$ mesons, as a function of rapidity 227 229]. Calculations within the framework of the higher-order MC@NLO pQCD model [230] are in reasonable agreement with the data although both the experimental and the theoretical systematic uncertainties are substantial again.

$\mathrm{B}^{+}$meson production in pp collisions was also investigated with the LHCb experiment 231 in the kinematic range $0<p_{\mathrm{t}}<40 \mathrm{GeV} / c$ and $2.0<y<4.5$. The measured $\mathrm{B}^{+}$cross sections are in good agreement with FONLL pQCD calculations [83]. Furthermore, the pseudorapidity dependence of bottom hadron production cross sections was measured with LHCb [232] in the range $2<\eta<6$ without an exclusive identification of the various hadron species. Also in this case, higher order pQCD calculations in the FONLL [83] and MCFM [233] frameworks are able to describe the measured data.

An alternative approach to bottom hadron production is the measurement of non-prompt $\mathrm{J} / \psi$ mesons originating from displaced bottom hadron decay vertices. All four large experiments at the LHC include vertex spectrometers with sufficient resolution for such measurements. The relative contribution $f_{\mathrm{B}}$ of bottom hadron decays to the inclusive $\mathrm{J} / \psi$ yield increases at midrapidity from $\approx 10 \%$ at low $\mathrm{J} / \psi p_{\mathrm{t}}$ to $\approx 2 / 3$ at high $p_{\mathrm{t}}$ as demonstrated in the upper left panel of Fig. 31 showing data from the ALICE [211], ATLAS 234], and CMS 235] experiments measured in pp collisions at $\sqrt{s}=7 \mathrm{TeV}$. The dashed line superimposed to the data indicates a semi-phenomenological function which is used to extrapolate the measured fraction down to zero $p_{\mathrm{t}}$. As an example, the ATLAS measurement of the $p_{\mathrm{t}}$-differential production cross section on non-prompt $\mathrm{J} / \psi$ mesons $[234$ is shown for four different rapidity intervals between mid-rapidity and $2.0<y_{\mathrm{J} / \psi}<2.4$ in the lower four panels of Fig. 31. Only in the rapidity interval $1.5<y_{\mathrm{J} / \psi}<2.0$ the ATLAS measurement reaches down to a $\mathrm{J} / \psi$ meson $p_{\mathrm{t}}$ of $1 \mathrm{GeV} / c$, whereas the minimum $p_{\mathrm{t}}$ in the other rapidity intervals is $5 \mathrm{GeV} / c$ or even higher. FONLL predictions are in agreement with the measured $p_{\mathrm{t}}$-differential production cross section for all rapidity selections. Furthermore, the measured rapidity dependence of the $\mathrm{J} / \psi$ cross section from bottom hadron decays is reproduced by FONLL within experimental and theoretical uncertainties as is demonstrated in the upper right panel of Fig. 31, which includes data from ALICE [211], CMS [235], and LHCb 236]. ALICE has measured the $p_{\mathrm{t}}$-differential cross section of non-prompt $\mathrm{J} / \psi$ meson production in the transverse momentum range $p_{\mathrm{t}}>1.3 \mathrm{GeV} / c$ at mid-rapidity $(|y|<0.9)$. The shape of the corresponding FONLL prediction was used to extrapolate the measurement to zero $p_{\mathrm{t}}$. A corresponding procedure was applied for the CMS measurement in the range $1.2<|y|<1.6$. The systematic uncertainties related to this extrapolation procedure are indicated by the slashed boxes in the upper right panel of Fig. 31 . Neither the CMS data in the interval $1.6<|y|<2.4$ nor the LHCb data had to be extrapolated since non-prompt $\mathrm{J} / \psi$ mesons have been measured down to zero $p_{\mathrm{t}}$ in these cases. The cross section measured with the LHCb experiment at forward rapidity (closed symbols) have been reflected around $y=0$ (open symbols). The corresponding FONLL calculation [83 and its uncertainty are indicated by the solid and dashed lines, respectively.

\subsubsection{Semielectronic heavy-flavor hadron decays}

Both ALICE and ATLAS have published first cross section measurements of electrons from semileptonic decays of heavy-flavor hadrons in pp collisions at $\sqrt{s}=7 \mathrm{TeV}$ at the LHC. The ALICE measurement 212$]$ is restricted to a narrow interval around mid-rapidity $(|y|<0.5)$. 

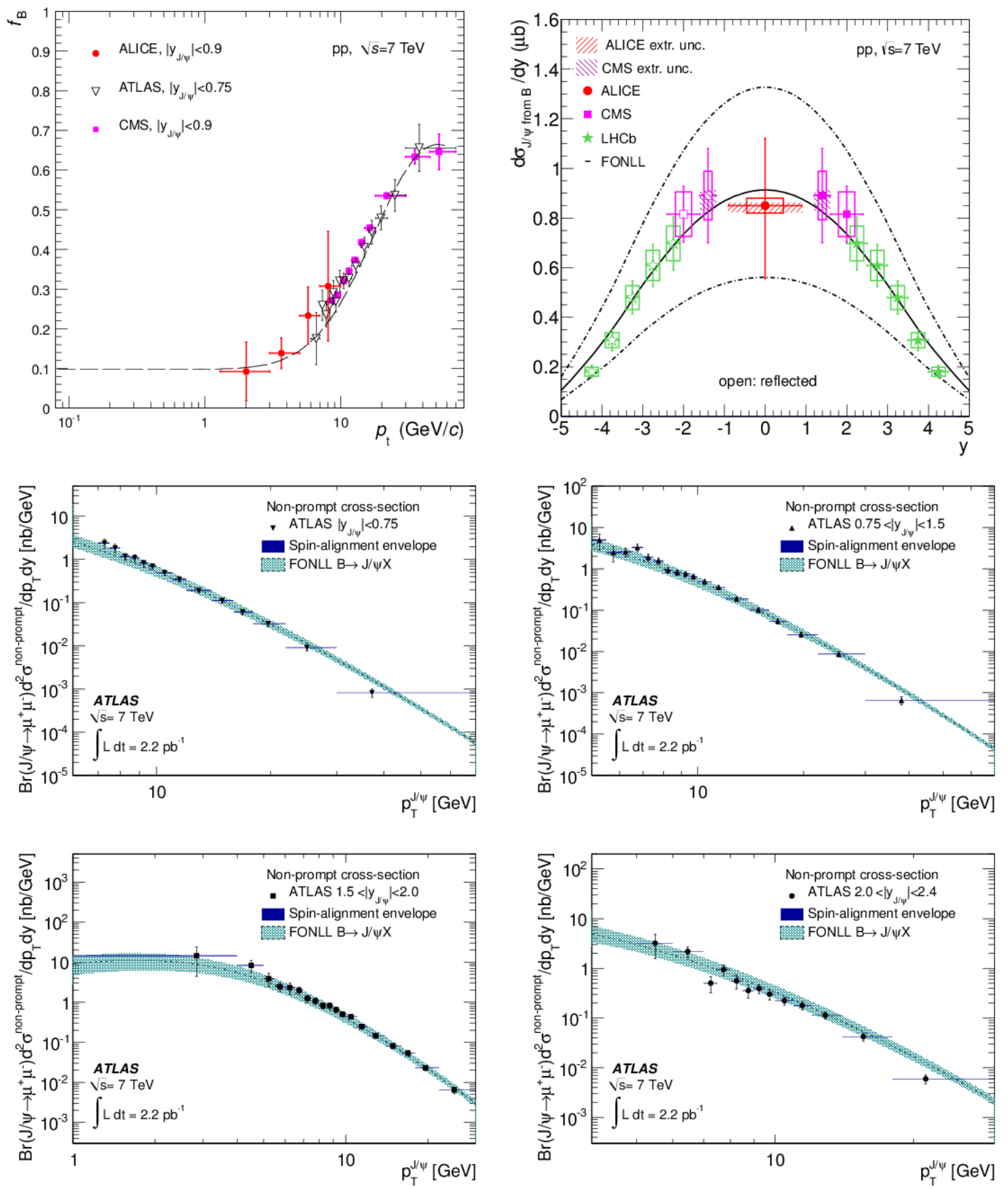

Figure 31: Fraction of $\mathrm{J} / \psi$ from the decay of bottom hadrons in the inclusive $\mathrm{J} / \psi$ sample as measured with ALICE [211], ATLAS [234], and CMS [235] in $7 \mathrm{TeV}$ pp collisions at mid-rapidity. Rapidity-differential production cross section of $\mathrm{J} / \psi$ from bottom-hadron decays measured with ALICE [211], CMS [235], and LHCb [236] (upper two panels, reprinted from Ref. 211] with kind permission from Springer Science and Business Media). $p_{\mathrm{t}}$-differential non-prompt $\mathrm{J} / \psi$ production cross sections in four rapidity intervals as measured with the ATLAS experiment (lower four panels, reprinted from Ref. 234]). FONLL pQCD predictions of $\mathrm{J} / \psi$ cross sections from bottom-hadron decays are compared with the measured differential $\mathrm{J} / \psi$ cross sections in all but the upper left panel. (see text for further details.) 

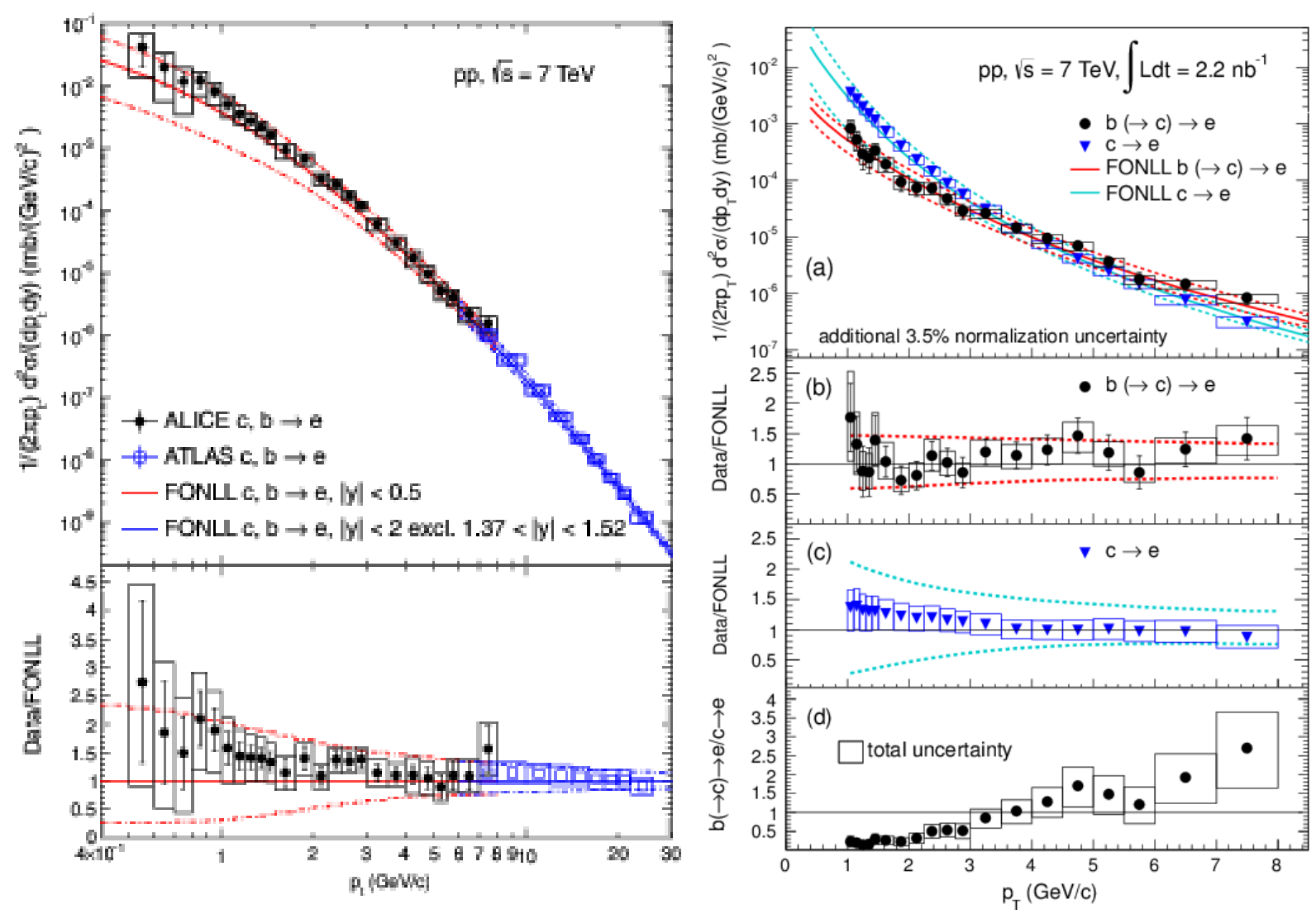

Figure 32: $p_{\mathrm{t}}$-differential production cross sections of electrons from heavy-flavor hadron decays measured around mid-rapidity with the ALICE [212] and ATLAS experiments [237] in pp collisions at $\sqrt{s}=7 \mathrm{TeV}$ (left panel, reprinted with permission from Ref. [212]; Copyright (2012) by the American Physical Society). $p_{\mathrm{t}}$-differential production cross sections of electrons from charm- and from bottom-hadron decays measured with ALICE at mid-rapidity in pp collisions at $\sqrt{s}=7 \mathrm{TeV}$ (right panel, reprinted from Ref. $[220]$ ). In both panels corresponding predictions from a FONLL pQCD calculation [83] are shown for comparison.

With ATLAS, electrons were reconstructed $[237]$ in the larger range $|y|<2$, excluding the interval $1.37<|y|<1.52$ in which the electron identification and the resolution of the energy measurement is degraded by a large amount of material in front of the first detector elements. The resulting $p_{\mathrm{t}}$-differential production cross sections, normalized per unit rapidity, are depicted in the left panel of Fig. 32. While the ALICE data include most of the total electron cross section from heavy-flavor hadron decays, the ATLAS data extend the measurement to higher $p_{\mathrm{t}}$. Exploiting the excellent vertexing capabilities of the ALICE ITS, electrons from charm and bottom hadron decays can be separated from each other in pp collisions at $\sqrt{s}=7 \mathrm{TeV}$ [220]. The resulting $p_{\mathrm{t}}$-differential production cross sections are shown in the right panel of Fig. 32 . Predictions from a FONLL pQCD calculation [83] are in good agreement both with the inclusive electrons cross sections from heavy-flavor hadron decays as well as with the individual cross sections of electrons from charm- and bottom-hadron decays, respectively, within experimental and theoretical uncertainties. 

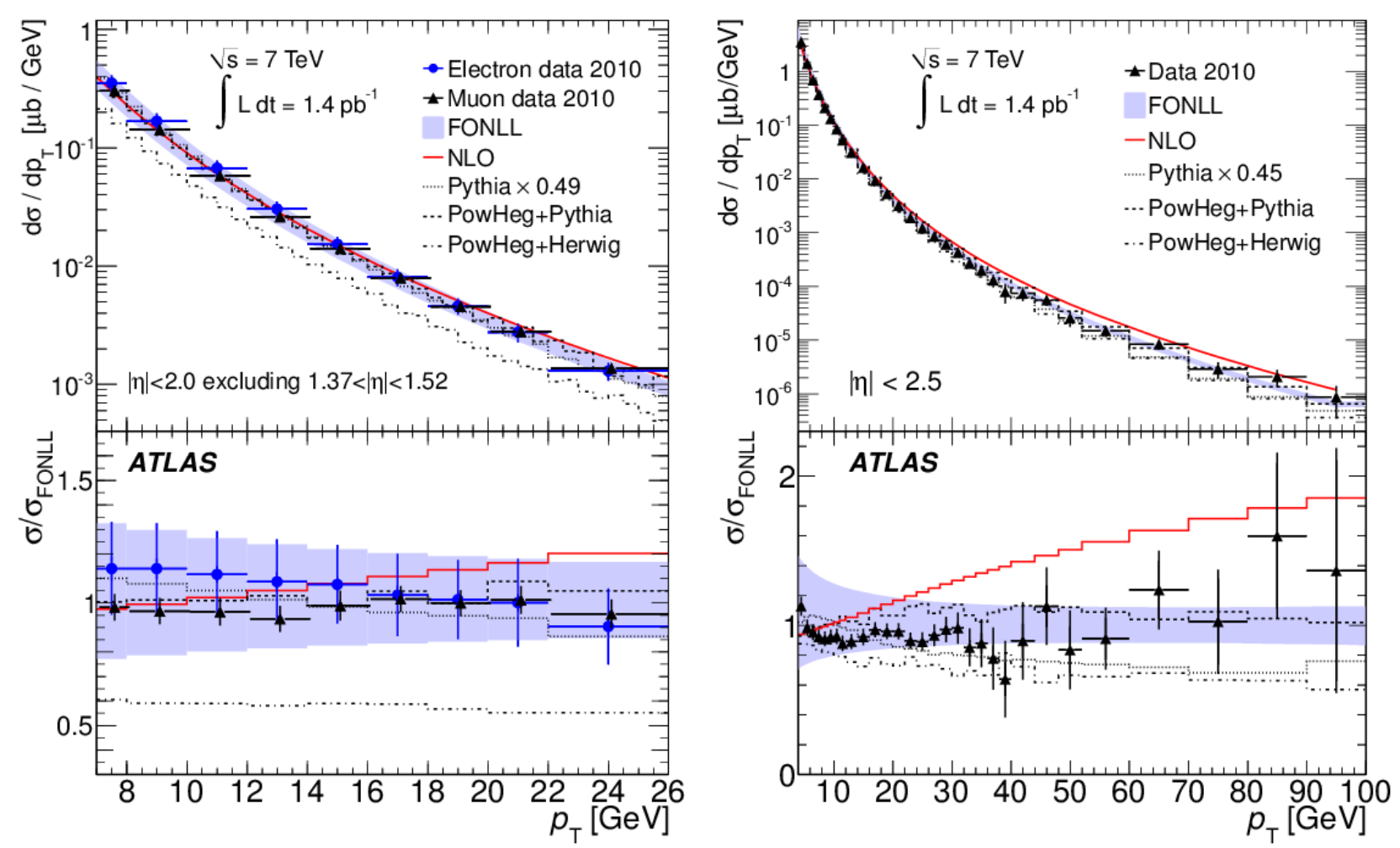

Figure 33: $p_{\mathrm{t}}$-differential production cross sections of leptons from heavy-flavor hadron decays measured with the ATLAS experiment in pp collisions at $\sqrt{S}=7 \mathrm{TeV}$ at the LHC. Electron and muon production cross sections for $|\eta|<2.0$, excluding the region $1.37<|\eta|<1.52$ (left). Muon production cross section for $|\eta|<2.5$ (right). Predictions from FONLL and other pQCD calculations are compared with the data. Ratios of the measured cross sections and of other predictions to the FONLL calculation are shown in the lower panels (see text for further details) (reprinted from Ref. [237]).

\subsubsection{Semimuonic heavy-flavor hadron decays}

Both with the ALICE [221] and ATLAS 237] experiments not only electrons but also muons from open heavy-flavor hadron decays have been measured at the LHC. The left panel of Fig. 33 shows a comparison of the muon $p_{\mathrm{t}}$-differential production cross section from ATLAS with the electron data (also shown in Fig. 32 for $p_{\mathrm{t}}>7 \mathrm{GeV} / c$ in the pseudorapidity range $|\eta|<2.0$, excluding the interval $1.37<|\eta|<1.52$ in which no electron measurement is available. In this high $p_{\mathrm{t}}$ range the mass difference between electrons and muons is irrelevant. Therefore, one would expect the cross sections of electrons and muons from semileptonic heavyflavor hadron decays to be equal which is confirmed within experimental uncertainties by the ATLAS data 237]. The data are confronted with various pQCD-based model calculations. The state-of-the are FONLL pQCD prediction [83], shown as a solid band in Fig. 33, agrees well with the data within experimental and theoretical uncertainties. In order to simplify the quantitative comparison of model predictions with the data the lower panels in Fig. 33 show the ratio of the data and the other model calculations to the FONLL prediction on a linear scale. The NLO calculation POWHEG [238,239] stops on the parton level and, therefore, has to be interfaced with parton shower generators to allow a comparison with decay lepton data. Here, 

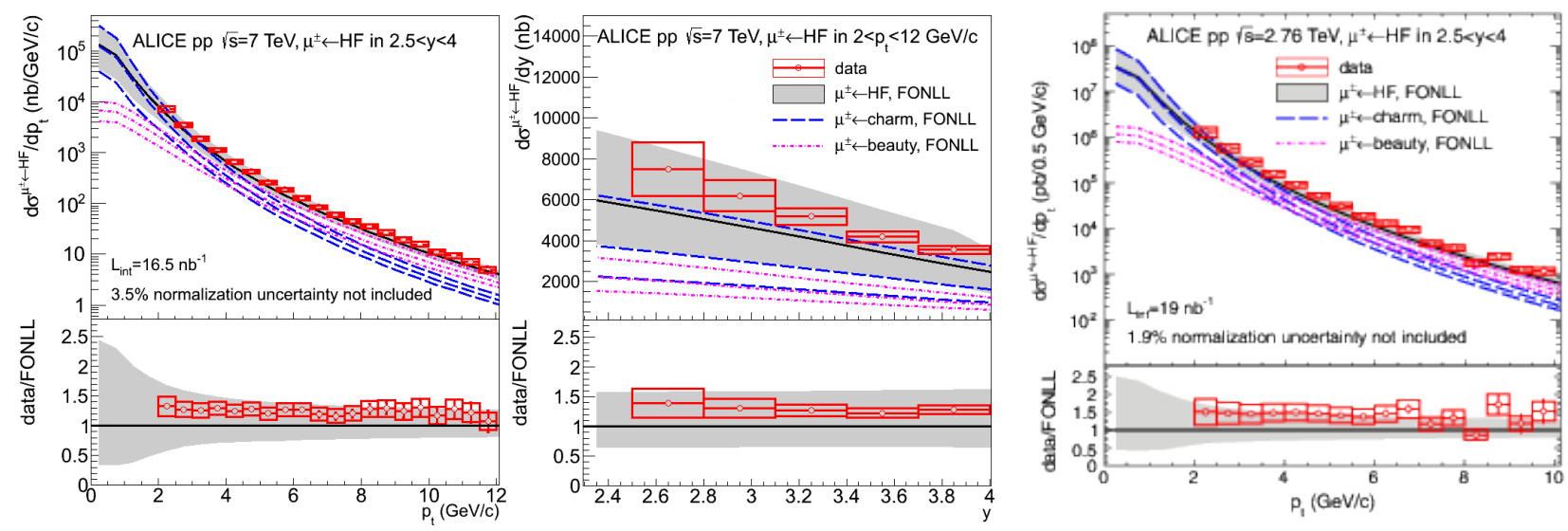

Figure 34: $p_{\mathrm{t}^{-}}$and rapidity-differential production cross sections of muons from heavy-flavor hadron decays measured with the ALICE experiment in the ranges $2<p_{\mathrm{t}}<12 \mathrm{GeV} / c$ and $-4<y<-2.5$ in pp collisions at $\sqrt{s}=7 \mathrm{TeV}$ (left and middle panel, reprinted from Ref. 221]), and $p_{\mathrm{t}}$-differential cross section at $\sqrt{s}=2.76 \mathrm{TeV}$ (right panel, reprinted with permission from Ref. [222]; Copyright (2012) by the American Physical Society) in comparison with predictions from FONLL pQCD calculations. Error bars (boxes) represent the statistical (systematic) uncertainties, where an additional 3.5\% absolute normalization uncertainty is not shown. Grey bands indicate the FONLL prediction 83] for muons from heavy-flavor hadron decays and the long dashed (short dashed) curves represent the FONLL predictions for charm (bottom) hadron decays separately. The ratios of the data to the FONLL prediction are shown in the lower panels on a linear scale.

PYTHIA [120] and HERWIG [240] have been used for the parton shower simulation. Whereas POWHEG+PYTHIA agree reasonably well with the data and with FONLL, this is different for POWHEG+HERWIG which predicts a significantly reduced lepton cross section. It was shown that this discrepancy can not be accounted for by the different hadron decay models. In addition, a LO PYTHIA pQCD calculation [120], which includes the parton shower simulation, predicts total cross sections approximately a factor two larger than the measurement, but with the proper shape of the decay lepton $p_{\mathrm{t}}$ distribution.

It is an interesting question whether the next-to-leading-log (NLL) resummation terms included in the FONLL pQCD calculation are actually important at the LHC or whether an NLO calculation without this resummation would be sufficient for the description of open heavyflavor data. In order to address this issue, an NLO central value prediction was calculated with the FONLL code excluding the NLL resummation [83]. An indication that this approach yields too hard lepton $p_{\mathrm{t}}$ spectra is visible in the left panel of Fig. 33 already, which shows the electron and muon $p_{\mathrm{t}}$ spectra up to $26 \mathrm{GeV} / c$. The right panel of Fig. 33 displays the $p_{\mathrm{t}}$-differential production cross section of muons from heavy-flavor hadron decays in the extended transverse momentum range up to $100 \mathrm{GeV} / c$ [237]. While the FONLL pQCD prediction and, to some extent, also the other pQCD calculations are in good agreement with the measurement, the NLO FONLL calculation ignoring the NLL resummation is clearly not consistent with data, demonstrating for the first time at a hadron collider the importance of the resummation of high order terms in the pQCD expansion.

At forward rapidity $(-4<y<-2.5)$ the $p_{\mathrm{t}^{-}}$and rapidity-differential production cross sections of muons from heavy-flavor hadron decays have been measured in pp collisions at $\sqrt{s}=$ 
$7 \mathrm{TeV} 221$ and $2.76 \mathrm{TeV} 222$ with the ALICE experiment as shown in Fig. 34 . Corresponding FONLL pQCD calculations are in good agreement with the measurement within the systematic uncertainties. In both semileptonic decay channels, i.e. electrons and muons, the FONLL pQCD calculations suggest that for $p_{\mathrm{t}}>5-6 \mathrm{GeV} / c$ the dominant contribution is from bottom hadron decays while at lower $p_{\mathrm{t}}$ charm hadron decays represent the relevant lepton source.

The correlation of single high- $p_{\mathrm{t}}$ muons with jets was measured with the CMS experiment in pp collisions at $\sqrt{s}=7 \mathrm{TeV}\left[241\right.$. Events with high- $p_{\mathrm{t}}$ muons $\left(p_{\mathrm{t}}^{\mu}>6 \mathrm{GeV} / c\right)$ have been selected in the range $\left|\eta^{\mu}\right|<2.1$. Events containing bottom hadrons were discriminated from background via the measurement of the muon transverse momentum with respect to the closest jet. The resulting $p_{\mathrm{t}^{-}}$and pseudorapidity-differential cross sections of muons from bottom-hadron decays have been confronted with pQCD calculations executed in the MC@NLO framework 230]. While the measured cross section are slightly larger than theoretically predicted, the data still agree with the model within the combined systematic uncertainties.

\subsubsection{FONLL-driven $\sqrt{s}$ scaling}

The heavy-flavor production cross sections measured in pp collisions at $\sqrt{s}=7 \mathrm{TeV}$ can be scaled to $\sqrt{s}=2.76 \mathrm{TeV}$ to provide a reference for the $\mathrm{Pb}+\mathrm{Pb}$ data at the same energy per nucleon-nucleon pair for cases in which a direct measurement of a pp reference at $2.76 \mathrm{TeV}$ is not available. This was demonstrated for the ALICE case in Ref. [242]. Since FONLL pQCD calculations [83 have been shown to be in reasonable agreement with all open heavy-flavor observables measured in pp collisions at $\sqrt{s}=7 \mathrm{TeV}$, FONLL was used for the necessary $\sqrt{s}$ scaling in this case. The scaling factors for D mesons, electrons, and muons were defined as the ratios of the corresponding cross sections from FONLL calculations at 2.76 and $7 \mathrm{TeV}$. The assumption was used that neither the factorization and renormalization scales nor the heavy-quark masses in the FONLL calculation vary with $\sqrt{s}$. To evaluate the uncertainties of the scaling factors, the scales and heavy-quark masses were varied over reasonable ranges and the envelopes of the resulting scaling factors were determined. For all open heavy-flavor observables the resulting uncertainties of the scaling functions are similar. They range from $\sim 25 \%$ at $p_{\mathrm{t}}=2 \mathrm{GeV} / c$ to less than $10 \%$ for $p_{\mathrm{t}}>10 \mathrm{GeV} / c$.

As mentioned above, with all LHC experiments pp data have been recorded at $\sqrt{s}=$ $2.76 \mathrm{TeV}$, albeit with limited statistics only. $p_{\mathrm{t}}$-differential muon spectra from heavy-flavor hadron decays have been measured with the ALICE experiment 222 as shown in the right panel of Fig. 34. Furthermore, D-meson $p_{\mathrm{t}^{-}}$differential production cross sections have been measured with ALICE at the same energy [243]. Both the muon and the D-meson data are in good agreement within uncertainties with the reference obtained via the FONLL-driven $\sqrt{s}$ scaling procedure from the $7 \mathrm{TeV}$ pp measurements to $2.76 \mathrm{TeV}$.

\subsection{Heavy flavor in $\mathrm{Pb}+\mathrm{Pb}$ collisions}

\subsubsection{Hadronic heavy-flavor hadron decays}

First results on open heavy-flavor production in $\mathrm{Pb}+\mathrm{Pb}$ collisions at $\sqrt{s_{\mathrm{NN}}}=2.76 \mathrm{TeV}$ at the LHC have been published recently. One of the major deficiencies of the measurements at RHIC has been overcome at the LHC right away. With ALICE, D mesons could be reconstructed even in central $\mathrm{Pb}+\mathrm{Pb}$ collisions 244 due to the excellent performance of the apparatus. Charged particle tracks are reconstructed in the ALICE TPC and ITS with a typical efficiency of $90 \%$. Furthermore, the ITS provides excellent vertexing precision, e.g. the track impact parameter 
resolution in the transverse plane is better than $50 \mu \mathrm{m}$ for $p_{\mathrm{t}} \geq 2 \mathrm{GeV} / c$. With the TOF system, which is instrumental for hadron identification, a time resolution in the order of 100 ps can be achieved. The D-meson transverse momentum spectra measured with ALICE in pp and $\mathrm{Pb}+\mathrm{Pb}$ collisions at the LHC contain feed down from bottom-hadron decays. This contribution was estimated based on the bottom production cross sections predicted from the FONLL calculation, which in pp collisions is in good agreement with the ALICE measurements of $\mathrm{J} / \psi$ and electrons from bottom-hadron decays as demonstrated in Figs. 31 and 32 , respectively. The feed down from bottom hadron decays was subtracted from the inclusive D-meson spectra resulting in the so-called prompt $\mathrm{D}$-meson yields. For $\mathrm{Pb}+\mathrm{Pb}$ collisions, the unknown nuclear modification factor of bottom hadrons introduces an additional systematic uncertainty on the prompt Dmeson yields in $\mathrm{Pb}+\mathrm{Pb}$ collisions. The conservative assumption was employed that the nuclear modification factor of bottom hadrons relative to the prompt D-meson $R_{\mathrm{AA}}$ fulfills the condition $0.3<R_{\mathrm{AA}}^{B} / R_{\mathrm{AA}}^{D}<3$. The resulting nuclear modification factors of prompt $\mathrm{D}^{0}, \mathrm{D}^{+}$, and $\mathrm{D}_{\mathrm{s}}^{+}$ mesons in the $20 \%$ most central $\mathrm{Pb}+\mathrm{Pb}$ collisions are shown in the upper left panel of Fig. 35 . A strong suppression is observed for all species reaching a factor 3-5 for $p_{\mathrm{t}}>8 \mathrm{GeV} / c$. Towards more peripheral $\mathrm{Pb}+\mathrm{Pb}$ collisions nuclear modification factors closer to one are measured for prompt $\mathrm{D}$ mesons as demonstrated for $40-80 \%$ central $\mathrm{Pb}+\mathrm{Pb}$ collisions in the upper right panel of Fig. 35. The centrality dependence of the D-meson $R_{\mathrm{AA}}$ is quantified in the middle panel of Fig. 35, which shows the nuclear modification factor of $\mathrm{D}^{0}$ mesons in the transverse momentum range $2<p_{\mathrm{t}}<5 \mathrm{GeV} / c$ as a function of the average number of participants $\left\langle N_{\text {part }}\right\rangle$ on the left and the same dependence for all D-meson species in the range $6<p_{\mathrm{t}}<12 \mathrm{GeV} / c$ on the right. Clearly the $\mathrm{D}$-meson suppression increases towards more central $\mathrm{Pb}+\mathrm{Pb}$ collisions.

It is an important issue whether the observed suppression of prompt D mesons at intermediate and high $p_{\mathrm{t}}$ might be related to shadowing of the parton distribution functions at the LHC. The comparison of an NLO pQCD calculation including the state of the art EPS09 parametrization of parton shadowing [245] with the measured D-meson $R_{\mathrm{AA}}$ as shown in the lower left panel of Fig. 35 provides evidence that this is not the case, i.e. the observed suppression clearly is a final state effect related to the hot and dense medium produced in $\mathrm{Pb}+\mathrm{Pb}$ collisions at the LHC. While parton shadowing seems not to be relevant for the interpretation of the prompt D-meson $R_{\mathrm{AA}}$, it is obvious that a highly desirable experimental confirmation of this conclusion will require data from proton-nucleus collisions at the LHC.

One of the most important experimental question left open by heavy-flavor measurements at RHIC is related to the expected mass hierarchy of hadron suppression. Radiative in-medium energy loss should be larger for light quark flavors compared to charm and, in particular, bottom quarks. However, the measured nuclear modification factor of electrons from heavyflavor hadron decays at high $p_{\mathrm{t}}$ was comparable in magnitude to the light-flavor dominated neutral pion $R_{\mathrm{AA}}$ in $\mathrm{Au}+\mathrm{Au}$ collisions at $\mathrm{RHIC}$, giving no hint of a quark mass hierarchy. The more exclusive measurements from $\mathrm{Pb}+\mathrm{Pb}$ collisions at the LHC might provide new insight into this issue. The nuclear modification factor of prompt D mesons measured [244] as a function of $p_{\mathrm{t}}$ with ALICE in the $20 \%$ most central $\mathrm{Pb}+\mathrm{Pb}$ collisions at mid-rapidity at the LHC is compared with corresponding ALICE measurements of charged hadrons [246] and non-prompt $\mathrm{J} / \psi$ mesons from bottom-hadron decays as measured with the CMS experiment 247] in the lower right panel of Fig. 35. While with the current statistical and systematic uncertainties a definite conclusion can not be drawn, the ALICE data hint towards a stronger suppression of charged hadrons relative to D mesons, as one would expect from a scenario in which induced gluon radiation is the dominant energy loss mechanism for partons propagating through a dense and color-charged medium. The nuclear modification factor of non-prompt $\mathrm{J} / \psi$ mesons 

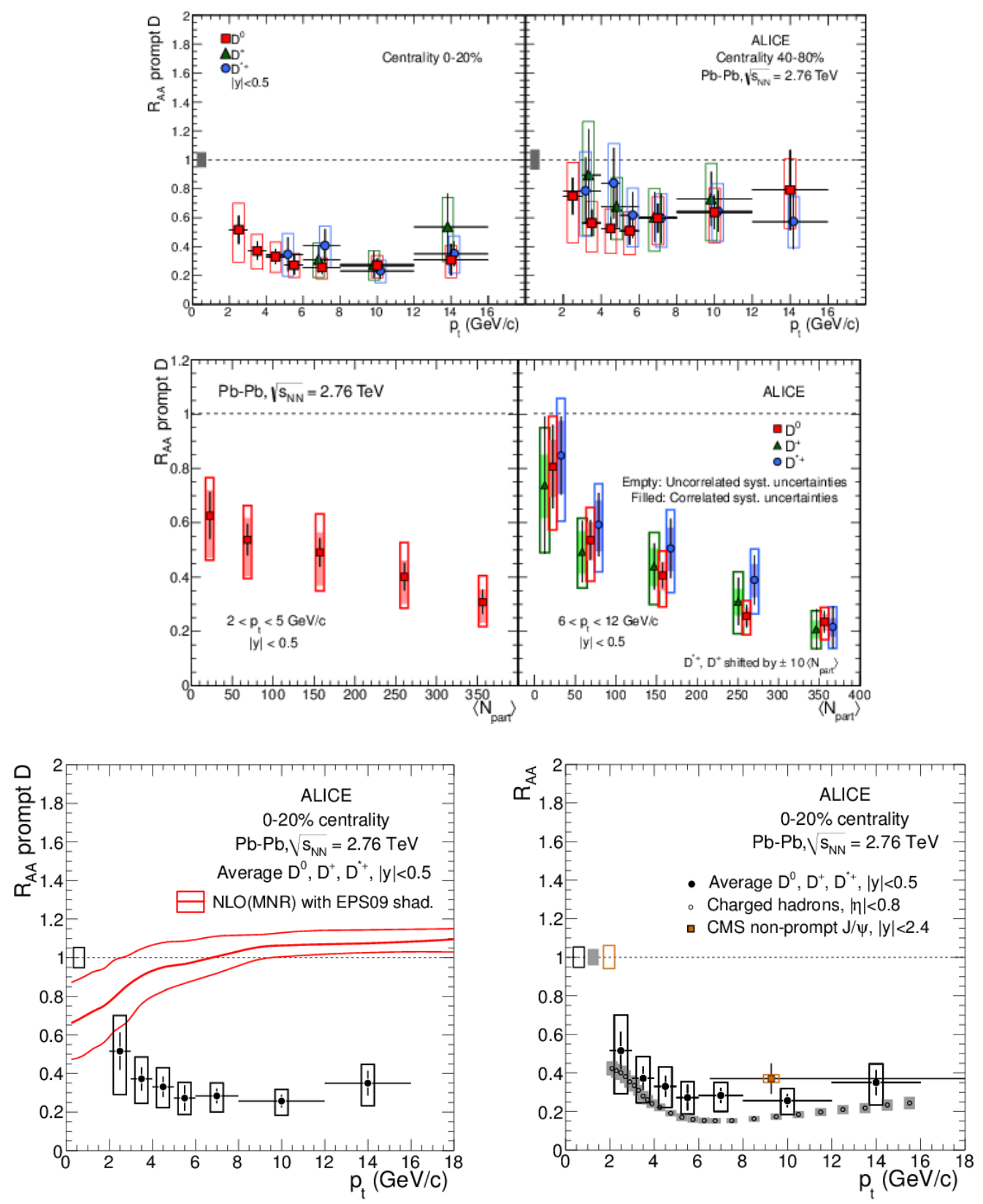

Figure 35: Nuclear modification factor $R_{\mathrm{AA}}$ of prompt $\mathrm{D}^{0}, \mathrm{D}^{+}$, and $\mathrm{D}_{\mathrm{s}}^{+}$mesons measured with the ALICE experiment in the $20 \%$ most central (upper left) and in $40-80 \%$ central (upper right) $\mathrm{Pb}+\mathrm{Pb}$ collisions at $\sqrt{s_{\mathrm{NN}}}=2.76 \mathrm{TeV}$. Error bars (boxes) show the statistical (systematic) uncertainties. The normalization uncertainties are indicated by the full boxes at one. Centrality dependence of $R_{\mathrm{AA}}$ for $\mathrm{D}^{0}$ mesons with $2<p_{\mathrm{t}}<5 \mathrm{GeV} / c$ (middle left) and all D-meson species with $6<p_{\mathrm{t}}<12 \mathrm{GeV} / c$ (middle right). Average D-meson $R_{\mathrm{AA}}$ in the $20 \%$ most central $\mathrm{Pb}+\mathrm{Pb}$ collisions in comparison with results from an NLO pQCD calculation including EPS09 nuclear shadowing [245] (lower left). D-meson $R_{\mathrm{AA}}$ compared with corresponding values of the nuclear modification factors of charged hadrons as measured with ALICE [246] and of non-prompt $\mathrm{J} / \psi$ mesons from bottom-hadron decays as measured with the CMS experiment 247] (lower right) (reprinted from Ref. 244] with kind permission from Springer Science and Business Media). 


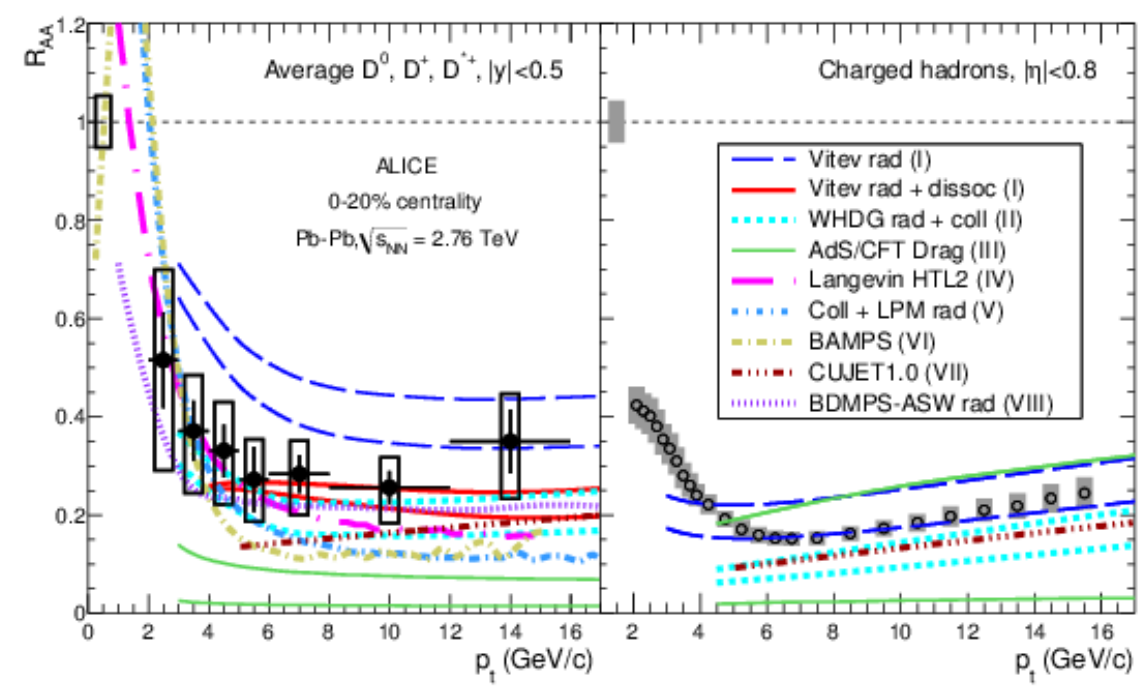

Figure 36: Average nuclear modification factor $R_{\mathrm{AA}}$ of various D-meson species measured as a function of $p_{\mathrm{t}}$ with the ALICE experiment at mid-rapidity in the $20 \%$ most central $\mathrm{Pb}+\mathrm{Pb}$ collisions at $\sqrt{s_{\mathrm{NN}}}=2.76 \mathrm{TeV}$ [244] (left panel) and the corresponding $R_{\mathrm{AA}}$ of charged hadrons in a slightly wider pseudoradidity interval [246] (right panel). Error bars (boxes) correspond to the statistical (systematic) uncertainties of the measurements. The normalization uncertainties, which are almost fully correlated for the two measurements, are indicated by the boxes around one. Predictions from various model calculations are compared to the data (see text for further details) (reprinted from Ref. 244 with kind permission from Springer Science and Business Media).

from bottom-hadron decays measured with the CMS experiment, clearly supports this picture because it is larger than the $R_{\mathrm{AA}}$ measured for charged hadrons. With CMS the $R_{\mathrm{AA}}$ of nonprompt $\mathrm{J} / \psi$ mesons from bottom-hadron decays was also measured in the $80 \%$ most peripheral collisions at $\sqrt{s_{\mathrm{NN}}}=2.76 \mathrm{TeV}$ 247]. Within uncertainties the measured value is consistent with the measurement in central $\mathrm{Pb}+\mathrm{Pb}$ collisions. In addition to the obvious necessity to collect statistically more significant data samples it is also mandatory to measure the nuclear modification factor of heavy- and light-flavor observables in proton-nucleus collisions at the LHC, such that cold nuclear matter effects can be separated from medium modifications due to the interaction of heavy quarks with the hot QCD matter produced in nuclear collisions at the LHC.

In the same context, it is important to confront theoretical predictions with the nuclear modification factors as measured in central $\mathrm{Pb}+\mathrm{Pb}$ collisions at the LHC. A comparison of various model predictions with the average $\mathrm{D}$ meson $R_{\mathrm{AA}}[244]$ (left panel) and with the $R_{\mathrm{AA}}$ of charged hadrons [246] (right panel) measured with the ALICE experiment in the 20\% most central $\mathrm{Pb}+\mathrm{Pb}$ collisions is shown in Fig. 36. In several models the nuclear modification factors are calculated both for D mesons and for charged hadrons. Among those, calculations which supplement radiative energy loss with the in-medium dissociation of D meson (curves I 103, 248 in Fig. 36) or with collisional energy loss via the WHDG (curves II [249]) or CUJET1.0 (curves VII [250|) implementations describe $R_{\mathrm{AA}}$ of $\mathrm{D}$ mesons and hadrons reasonably well at the same time. In the model incorporating in-medium formation and dissociation of $\mathrm{D}$ mesons the 


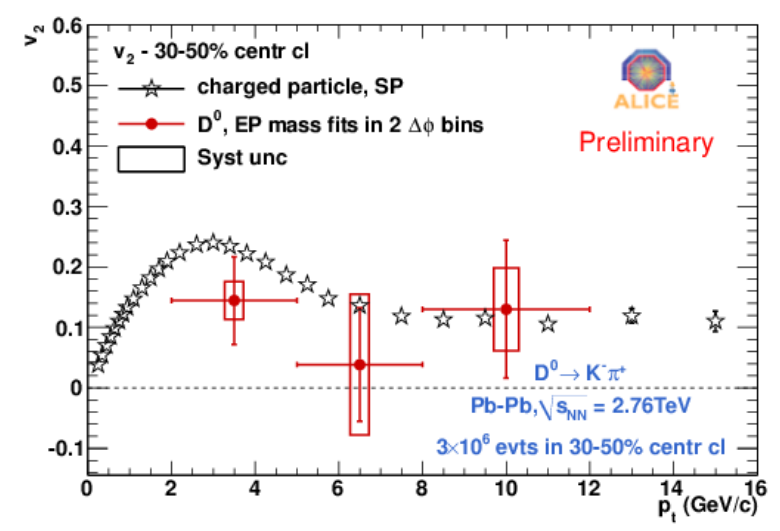

Figure 37: Elliptic-flow strength $v_{2}$ of $\mathrm{D}^{0}$ mesons measured with the ALICE experiment 256 as a function of $p_{\mathrm{t}}$ in mid-central $\mathrm{Pb}+\mathrm{Pb}$ collisions in comparison with the corresponding $v_{2}$ of unidentified charged particles [65] (reprinted from Ref. [256]).

medium density is tuned such that it describes the inclusive jet suppression measured at the LHC. This is different for the WHDG and CUJET1.0 calculations which extrapolate the medium density required to describe the pion suppression observed at RHIC to LHC conditions. This extrapolation might contribute to the fact that the nuclear modification factors calculated for charged hadrons in these two models are slightly smaller than measured. The average D-meson $R_{\mathrm{AA}}$ calculated in a completely different model approach based on AdS/CFT drag coefficient (curves III [104]) underestimates the measurement significantly. The predictive power for the charged hadron $R_{\mathrm{AA}}$ is only limited in this model. Other models, including a Langevin transport calculation (curve IV [251,252]), a calculation including collisional and radiative energy loss in the medium (curve V 196 253]), a pQCD based partonic transport calculation (curve VI [254]), and a calculation employing radiative energy loss only (curve VIII [255]), provide the average D-meson $R_{\mathrm{AA}}$ only. In general, these model calculations are in reasonable agreement with the data with a tendency to underpredict the measured D-meson $R_{\mathrm{AA}}$.

The measurement of nuclear modification factors of $\mathrm{D}$ mesons demonstrates the strong interaction of heavy quarks produced in the earliest phase of $\mathrm{Pb}+\mathrm{Pb}$ collisions at the $\mathrm{LHC}$ with the hot and dense partonic medium that is formed afterwards. It is an important question whether heavy quarks participate in the collective expansion of this medium and whether they might thermalize. This question can be addressed via the measurement of the elliptic flow strength $v_{2}$ of particles carrying heavy quarks. $v_{2}$ was measured for neutral D mesons [256] in mid-central $\mathrm{Pb}+\mathrm{Pb}$ collisions with the ALICE experiment as shown in Fig. 37. A correction for feed down from B-meson decays was not applied yet. While statistical and systematic uncertainties are still large, an indication for a non-zero $v_{2}$ of $\mathrm{D}$ mesons is observed. Clearly, more statistics is necessary to be able to evaluate the centrality dependence of D-meson flow, and it will be important to reduce the systematic uncertainties before any strong conclusion can be drawn.

\subsubsection{Semileptonic heavy-flavor hadron decays}

Both electrons and muons from heavy-flavor hadron decays have been measured with the ALICE experiment in $\mathrm{Pb}+\mathrm{Pb}$ collisions at $\sqrt{s_{\mathrm{NN}}}=2.76 \mathrm{TeV}$. The electron analysis in the $p_{\mathrm{t}}$ range up 

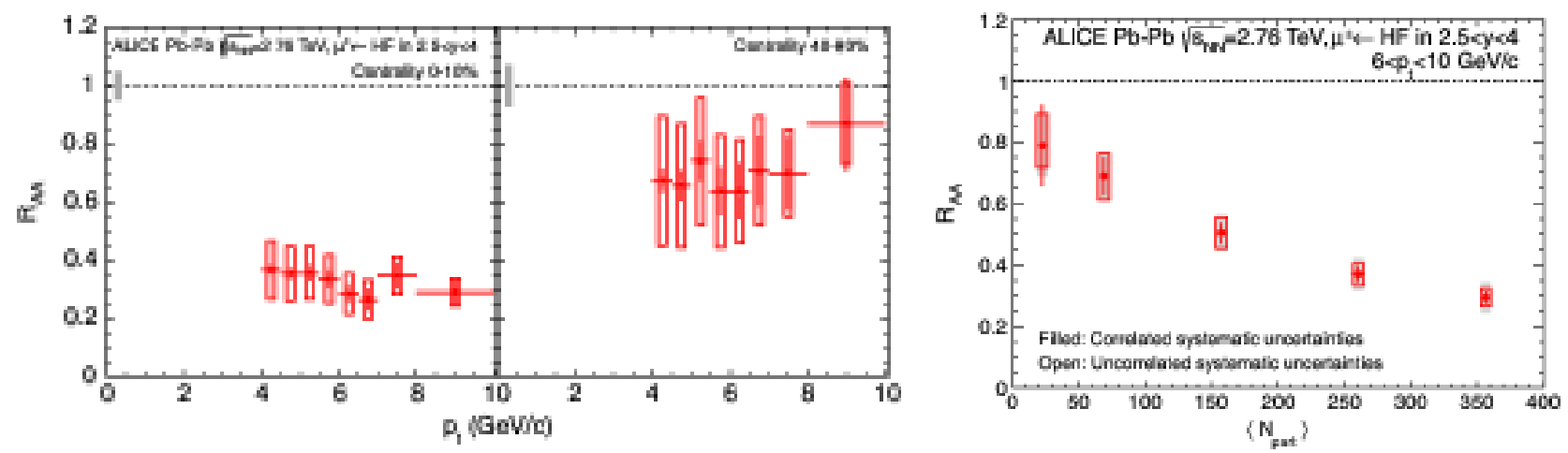

Figure 38: Nuclear modification factor of muons from heavy-flavor hadron decays measured as a function of $p_{\mathrm{t}}$ with the ALICE experiment in the rapidity range $2.5<y<4.0$ in the $10 \%$ most central and in $40-80 \%$ central $\mathrm{Pb}+\mathrm{Pb}$ collisions (left panel). Centrality dependence of the muon $R_{\mathrm{AA}}$ as a function of the average number of participating nucleons in the ranges $2.5<y<4.0$ and $4<p_{\mathrm{t}}<10 \mathrm{GeV} / c$ (right panel) (reprinted with permission from Ref. 222]; Copyright (2012) by the American Physical Society).

to 6g̃evc at mid-rapidity $(|y|<0.5)$ is currently being finalized. Preliminary results 257] indicate a strong suppression in central collisions consistent with the suppression observed for muons from heavy-flavor hadron decays (see below) although the systematic uncertainties are still large. Towards more peripheral collisions the high- $p_{\mathrm{t}}$ heavy-flavor electron suppression decreases, again consistent with the muon case.

For muons from heavy-flavor hadron decays the nuclear modification factors as measured with the ALICE experiment 222$]$ at forward rapidity $(2.5<y<4.0)$ in the $10 \%$ most central and $40-80 \%$ central collisions are presented as a function of $p_{\mathrm{t}}$ in the left panel of Fig. 38. In contrast to the D-meson $R_{\mathrm{AA}}$ measurement the muon pp reference could be taken directly from the ALICE pp measurement at $\sqrt{s}=2.76 \mathrm{TeV}$ and not from the FONLL-driven $\sqrt{s}$ scaling [242 of the pp data at $7 \mathrm{TeV}$. This was possible only because a hardware muon candidate trigger allowed a significant muon sample to be recorded in the relatively short pp run period of the $\mathrm{LHC}$ at $2.76 \mathrm{TeV}$. In the transverse momentum range $4<p_{\mathrm{t}}<10 \mathrm{GeV} / c$ a strong suppression of muons from heavy-flavor hadron decays is observed in the most central $\mathrm{Pb}+\mathrm{Pb}$ collisions without a pronounced $p_{\mathrm{t}}$ dependence. In $40-80 \%$ central $\mathrm{Pb}+\mathrm{Pb}$ collisions a suppression is observed as well, although to a much lesser extent, again without any significant $p_{\mathrm{t}}$ dependence. The pronounced centrality dependence of the muon $R_{\mathrm{AA}}$ from heavy-flavor hadron decays is quantified in the right panel of Fig. 38 which presents the nuclear modification factor of such muons measured with ALICE in the range $6<p_{\mathrm{t}}<10 \mathrm{GeV} / c$ at forward rapidity as a function of the average number of participating nucleons in various $\mathrm{Pb}+\mathrm{Pb}$ centrality classes. While in peripheral $\mathrm{Pb}+\mathrm{Pb}$ collisions only a small suppression is observed, it increases strongly towards central collisions. However, it is important to note that for the decay muons in the $p_{\mathrm{t}}$ range investigated here, a significant fraction originates from the decay of bottom hadrons. Therefore, these data emphasize again (see also the lower right panel of Fig. 35) that not only charm but also bottom quarks are coupled strongly to the hot and dense QCD medium produced in highenergy heavy-ion collisions. 


\section{Summary and outlook}

Hadrons carrying heavy flavor, i.e. charm or bottom quarks, have been shown to be among the most interesting probes in modern particle and nuclear physics. Because of the large quark masses heavy-flavor production proceeds mainly through hard parton-parton scattering processes in the earliest stage of hadronic collisions. As such the measurement of heavy-flavor production in pp collisions provides a crucial testing ground for perturbative QCD calculations. State of the art FONLL pQCD calculations are in agreement with all of such measurements within statistical and systematic uncertainties at the Fermilab Tevatron, the BNL RHIC, and at the CERN LHC. Furthermore, these measurements serve as a baseline for heavy-flavor studies in nucleus-nucleus collisions, in which the heavy quarks propagate through and interact with the hot and dense medium produced in the nuclear collisions. The investigation of in-medium modifications of heavy-flavor observables can shed light on the properties of the QCD medium and the nature of parton-medium interactions. This is the case both for heavy-quarkonia, which have not been discussed in this review (see Ref. [80] instead) and for which open heavy-flavor measurements can provide a natural baseline, and for open heavy-flavor observables, which have been in the focus of this review.

While extensive systematic studies of the production of heavy quarkonia have been conducted in the nucleus-nucleus collision program at the CERN SPS, the production of open heavy-flavor hadrons does not play a role in the collision dynamics in this rather low energy regime.

First systematic studies of open heavy-flavor production in nucleus-nucleus collisions have been conducted at RHIC, mainly in the semi-electronic decay channel, where it was verified that the total heavy-flavor yields scale with the number of binary collisions. Measurements of the nuclear modification factor and of the elliptic flow strength of electrons from heavyflavor hadron decays demonstrate the strong coupling of heavy quarks with the hot and dense QCD medium produced in $\mathrm{Au}+\mathrm{Au}$ collisions at RHIC. To further elucidate the properties of hot QCD matter it is important to shed light on the relevant interaction mechanism of heavy quarks with the medium. It is still not clear which mechanisms are finally responsible for the strong suppression observed for high $p_{\mathrm{t}}$ electrons in central $\mathrm{Au}+\mathrm{Au}$ collisions at RHIC.

At the CERN LHC, the systematic study of open heavy-flavor production in nucleus-nucleus collisions is continued in an unprecedented high-energy regime. In addition, for the first time, exclusive charm and bottom measurements have become possible in nucleus-nucleus collisions thanks to the availability of silicon vertex spectrometers that allow the separation of secondary, displaced heavy-flavor hadron decay vertices from the primary collision vertex. The general picture established at RHIC was confirmed by the first measurements at the LHC already, but major additional progress is expected in the near future through the systematic study of the centrality, $p_{\mathrm{t}}$, and rapidity dependence of exclusive charm and bottom observables both in $\mathrm{Pb}+\mathrm{Pb}$ and soon in $\mathrm{p}+\mathrm{Pb}$ collisions. Table 3 gives an overview over the measured open heavy-flavor channels and their kinematic coverage at RHIC and at the LHC until July 2012. It should be noted that since then preliminary results have become available, in particular from the LHC, which significantly extend the kinematic coverage in most channels.

The future is bright for open heavy-flavor measurements in high-energy nucleus-nucleus collisions. The experimental program at the LHC has just begun. At RHIC, after the very successful first decade of operations at the world's first dedicated heavy-ion collider, the accelerator is upgraded to deliver higher luminosities and concurrently the PHENIX and STAR detectors are undergoing major upgrade programs. Key elements of these upgrades are silicon 
Table 3: Overview over measurements related to open heavy-flavor production conducted at RHIC $\left(\sqrt{s_{\mathrm{NN}}} \leq 0.2 \mathrm{TeV}\right)$ and at the LHC $\left(\sqrt{s_{\mathrm{NN}}} \leq 7 \mathrm{TeV}\right)$ until July 2012. For each collision system, center-of-mass energy $\sqrt{s_{\mathrm{NN}}}$, and channel investigated the phase space coverage is given as well.

\begin{tabular}{|c|c|c|c|c|c|}
\hline system & $\sqrt{s_{\mathrm{NN}}}[\mathrm{TeV}]$ & channel & $p_{\mathrm{t}}$ coverage $[\mathrm{GeV} / c]$ & $y$ coverage & ref. \\
\hline \multirow[t]{10}{*}{$\mathrm{pp}$} & 0.2 & $\mathrm{D}^{0}$ & $0.6<p_{\mathrm{t}}<2.0$ & $|y|<1$ & [170] \\
\hline & & $\mathrm{D}^{*}$ & $2<p_{\mathrm{t}}<6$ & $|y|<1$ & 170 \\
\hline & & $c, b \rightarrow e^{ \pm}$ & $0.3<p_{\mathrm{t}}<9.0$ & $|y|<0.35$ & 155 \\
\hline & & $c, b \rightarrow e^{ \pm}$ & $1.2<p_{\mathrm{t}}<10.0$ & $|y|<1$ & 174 \\
\hline & & $c \rightarrow e^{ \pm}$ & $2<p_{\mathrm{t}}<7$ & $|y|<0.35$ & 163 \\
\hline & & $b \rightarrow e^{ \pm}$ & $2<p_{\mathrm{t}}<7$ & $|y|<0.35$ & 163 \\
\hline & & $c \rightarrow e^{ \pm}$ & $2.5<p_{\mathrm{t}}<9.5$ & $|y|<0.7$ & 175 \\
\hline & & $b \rightarrow e^{ \pm}$ & $2.5<p_{\mathrm{t}}<9.5$ & $|y|<0.7$ & 175 \\
\hline & & $c, b \rightarrow e^{+} e^{-}$ & $p_{t}^{e}>0.2$ & $|y|<0.35$ & 164,165 \\
\hline & & $c, b \rightarrow \mu^{-}$ & $1<p_{\mathrm{t}}<7$ & $1.4<|y|<1.9$ & 156 \\
\hline \multirow[t]{3}{*}{$\mathrm{d}+\mathrm{Au}$} & 0.2 & $\mathrm{D}^{0}$ & $0.1<p_{\mathrm{t}}<3.0$ & $|y|<1$ & 169 \\
\hline & & $c, b \rightarrow e^{ \pm}$ & $0.85<p_{\mathrm{t}}<8.5$ & $|y|<0.35$ & 181 \\
\hline & & $c, b \rightarrow e^{ \pm}$ & $1<p_{\mathrm{t}}<10$ & $|y|<1$ & 173 \\
\hline $\mathrm{Cu}+\mathrm{Cu}$ & 0.2 & $c, b \rightarrow \mu^{-}$ & $1<p_{\mathrm{t}}<4$ & $1.4<|y|<1.9$ & {$[156$} \\
\hline $\mathrm{Au}+\mathrm{Au}$ & 0.13 & $c, b \rightarrow e^{ \pm}$ & $0.5<p_{\mathrm{t}}<3.0$ & $|y|<0.35$ & {$[157]$} \\
\hline \multirow[t]{4}{*}{$\mathrm{Au}+\mathrm{Au}$} & 0.2 & $\mathrm{D}^{0}$ & $p_{\mathrm{t}}<2.0$ & $|y|<1$ & 171 \\
\hline & & $c, b \rightarrow e^{ \pm}$ & $0.3<p_{\mathrm{t}}<9.0$ & $|y|<0.35$ & 155 \\
\hline & & $c, b \rightarrow e^{ \pm}$ & $1.2<p_{\mathrm{t}}<10.0$ & $|y|<1$ & 173 \\
\hline & & $c, b \rightarrow e^{+} e^{-}$ & $p_{t}^{e}>0.2$ & $|y|<0.35$ & 165 \\
\hline \multirow[t]{14}{*}{$\mathrm{pp}$} & 7.0 & $\mathrm{D}^{0}, \mathrm{D}^{+}, \mathrm{D}^{*+}$ & $\begin{array}{l}\mathrm{D}^{+}, \mathrm{D}^{*+}: 1<p_{\mathrm{t}}<24 \\
\mathrm{D}^{0}: 1<p_{\mathrm{t}}<16\end{array}$ & $|y|<0.5$ & $|210|$ \\
\hline & & $\mathrm{B}^{0}, \mathrm{~B}^{+}, \mathrm{B}_{\mathrm{s}}^{0}$ & $\begin{array}{l}\mathrm{B}^{+}, \mathrm{B}^{0}: p_{\mathrm{t}}>5 \\
\mathrm{~B}_{\mathrm{s}}^{0}: p_{\mathrm{t}}>5\end{array}$ & $\begin{array}{l}\mathrm{B}^{+}, \mathrm{B}_{\mathrm{s}}^{0}:|y|<2.4 \\
\mathrm{~B}^{0}:|y|<2.2\end{array}$ & $227-229$ \\
\hline & & $\mathrm{B}^{+}$ & $0<p_{\mathrm{t}}<40$ & $2.0<y<4.5$ & 231 \\
\hline & & $\mathrm{b}$ hadron $\rightarrow \mathrm{J} / \psi$ & $p_{\mathrm{t}}>0$ & $|y|<0.9$ & 211 \\
\hline & & $\mathrm{b}$ hadron $\rightarrow \mathrm{J} / \psi$ & $7<p_{\mathrm{t}}<70$ & $|y|<0.75$ & 234 \\
\hline & & $\mathrm{b}$ hadron $\rightarrow \mathrm{J} / \psi$ & $1<p_{\mathrm{t}}<30$ & $1.5<|y|<2.0$ & 234 \\
\hline & & $\mathrm{b}$ hadron $\rightarrow \mathrm{J} / \psi$ & $p_{\mathrm{t}}>6.5$ & $|y|<1.2$ & 235 \\
\hline & & $\mathrm{b}$ hadron $\rightarrow \mathrm{J} / \psi$ & $p_{\mathrm{t}}>0$ & $1.6<|y|<2.4$ & 235 \\
\hline & & $c, b \rightarrow e^{ \pm}$ & $0.5<p_{\mathrm{t}}<8.0$ & $|y|<0.5$ & 212 \\
\hline & & $c, b \rightarrow e^{ \pm}$ & $7<p_{\mathrm{t}}<26$ & $|y|<2.0$ & 237 \\
\hline & & $c \rightarrow e^{ \pm}$ & $1<p_{\mathrm{t}}<8$ & $|y|<0.8$ & 220 \\
\hline & & $b \rightarrow e^{ \pm}$ & $1<p_{\mathrm{t}}<8$ & $|y|<0.8$ & 220 \\
\hline & & $c, b \rightarrow \mu^{-}$ & $2<p_{\mathrm{t}}<12$ & $2.5<y<4.0$ & 221 \\
\hline & & $c, b \rightarrow \mu^{-}$ & $7<p_{\mathrm{t}}<24$ & $|y|<2.0$ & 237 \\
\hline \multirow[t]{2}{*}{$\mathrm{pp}$} & 2.76 & $\mathrm{D}^{0}, \mathrm{D}^{+}, \mathrm{D}^{*+}$ & $1<p_{\mathrm{t}}<12$ & $|y|<0.5$ & 243 \\
\hline & & $c, b \rightarrow \mu^{ \pm}$ & $2<p_{\mathrm{t}}<10$ & $2.5<y<4.0$ & 222 \\
\hline \multirow[t]{4}{*}{$\mathrm{Pb}+\mathrm{Pb}$} & 2.76 & $\mathrm{D}^{0}, \mathrm{D}^{+}, \mathrm{D}^{*+}$ & $2<p_{\mathrm{t}}<16$ & $|y|<0.5$ & 243 \\
\hline & & $\mathrm{b}$ hadron $\rightarrow \mathrm{J} / \psi$ & $6.5<p_{\mathrm{t}}<30$ & $|y|<2.4$ & 247 \\
\hline & & $c, b \rightarrow e^{ \pm}$ & $3.5<p_{\mathrm{t}}<6.0$ & $|y|<0.5$ & 257 \\
\hline & & $c, b \rightarrow \mu^{ \pm}$ & $4<p_{\mathrm{t}}<10$ & $2.5<|y|<4.0$ & 2 \\
\hline
\end{tabular}


vertex detectors aimed towards the separation of displaced heavy-flavor hadron decay vertices from the primary collision vertex. With these upgrades in place it will be possible both at RHIC [152] as well as at the LHC to measure the yields and phase space distributions of charm and bottom hadrons in pp, p/d-nucleus, and nucleus-nucleus collisions via exclusive channels, providing unique hard probes for hot QCD matter even at low $p_{\mathrm{t}}$. In particular the measurement of heavy-flavor baryons, notably the $\Lambda_{\mathrm{c}}$, would be important even though this is very challenging, markedly in nucleus-nucleus collisions.

Precision measurements of the nuclear modification factors of heavy-flavor hadrons will address the issue whether the expected mass ordering of quark energy loss in the hot and dense medium is realized in nature or not. This is one of the crucial questions related to the in-medium parton dynamics and, in particular, to the mechanism of parton energy loss in the medium. The latter is up to now not understood on a fundamental level. Tightly connected to the energy loss of a hard probe is the reaction of the medium to the resulting energy deposit. Regarding these issues, heavy-flavor measurements can contribute a unique piece of information. With the high resolution vertex detectors at RHIC and at the LHC it should be possible to "tag" charm and, in particular, bottom jets on an event-by-event basis. In contrast to high- $p_{\mathrm{t}}$ particle production, which at RHIC and at the LHC is dominated by gluon jets, such heavyflavor tags give clean access to quark jets. Furthermore, momentum conservation in the hard scattering process gives rise to a back-to-back correlation in azimuth of the quark and antiquark jets produced associately. Therefore, tagged heavy-quark jets constitute a new quality of hard probes as they give much better access to the kinematics of the initially scattered partons and allow the investigation of the mass dependence of parton energy loss and the medium response. Also, the precision measurement of heavy-flavor hadron flow will be possible at RHIC and at the LHC, such that the question can be addressed to what extent heavy quarks reach equilibrum with the hot and dense medium. This is a crucial issue as it is directly related to the transport properties of the medium.

With precision vertexing it should furthermore be possible to measure at RHIC and at the LHC the contribution of correlated semileptonic heavy-flavor hadron decays to the dilepton continuum in the intermediate mass region, which at RHIC is dominated by charm hadron decays while at the LHC bottom hadron decays contribute significantly. In nucleus-nucleus collisions, these dileptons carry information about the interaction of heavy quarks with the medium in a twofold way. First, the quarks lose energy in the medium and second the opening angle between the quark and the antiquark can be modified. In the extreme case, the heavy quarks thermalize with the medium such that the angular correlation between the quark and antiquark is completely lost. Again, a comparison of charm and bottom measurements would be useful regarding the issue of the parton energy loss mechanism and it could help to better understand the transport properties of the medium. Furthermore, it should be noted that only two sources are expected to contribute significantly to the intermediate mass dilepton continuum, i.e. correlated heavy-flavor hadron decays and thermal radiation from the hot medium. If these two contributions can be disentangled with the help of precision vertexing the temperature of the emitting source and its evolution with time can be investigated in a unique way.

In the longer term future, open charm production will be addressed at the FAIR facility currently under construction at GSI. At this fixed-target machine charm can be investigated close to the production threshold in proton-nucleus and nucleus-nucleus collisions, where models predict in-medium effects which in a unique way could shed light on the properties of the baryon-rich form of dense QCD matter produced at FAIR [129]. 


\section{References}

[1] H. Fritzsch, M. Gell-Man, and H. Leutwyler Phys. Lett. B 47 (1973) 365

[2] N. Itoh, Prog. Theor. Phys. 44 (1970) 291

[3] N. Cabibbo and G. Parisi, Phys. Lett. B 59 (1975) 67

[4] J.C. Collins and M.J. Perry, Phys. Rev. Lett. 34 (1975) 1353

[5] K.G. Wilson, Phys. Rev. D 10 (1974) 2445

[6] M. Creutz, Phys. Rev. D 15 (1975) 1128

[7] E. Laermann and O. Philipsen, Ann. Rev. Nucl. Part. Sci. 53 (2003) 163

[8] Z. Fodor and S.D. Katz, JHEP 0404 (2004) 050

[9] S. Ejiri et al., Prog. Theor. Phys. 153 (2004) 118

[10] Y. Aoki et al., Nature 443 (2006) 675

[11] Y Aoki et al., Phys. Lett. B 643 (2006) 46

[12] M. Cheng et al., Phys. Rev. D 74 (2006) 054507

[13] A. Bazavov et al., Phys. Rev. D 80 (2009) 014504

[14] D.H. Rischke, Prog. Part. Nucl. Phys. 52 (2004) 197

[15] M. Buballa, Phys. Rep. 407 (2005) 205

[16] M.G. Alford et al., Rev. Mod. Phys. 80 (2008) 1455

[17] F. Weber, Prog. Part. Nucl. Phys. 54 (2005) 193

[18] N.A. Gentile et al., Astrophys. J. 414 (1993) 701

[19] I. Sagert et al., Phys. Rev. Lett. 102 (2009) 081101

[20] U. Heinz and M. Jacob, arXiv:nucl-th/0002042

[21] I. Arsene et al. (BRAHMS Collaboration), Nucl. Phys. A 757 (2005) 1

[22] K. Adcox et al. (PHENIX Collaboration), Nucl. Phys. A 757 (2005) 184

[23] B. Back et al. (PHOBOS Collaboration), Nucl. Phys. A 757 (2005) 28

[24] J. Adams et al. (STAR Collaboration), Nucl. Phys. A 757 (2005) 102

[25] S. Voloshin and Y. Zhang, Z. Phys. C 70 (1996) 665

[26] A.P. Mishra et al., Phys. Rev. C 77 (2008) 064902

[27] A.P. Mishra et al., Phys. Rev. C 81 (2010) 034903

[28] J. Takahashi et al., Phys. Rev. Lett. 103 (2009) 242301

[29] B. Alver and G. Roland, Phys. Rev. C 81 (2010) 054905

[30] B.H. Alver et al., Phys. Rev. C 82 (2010) 034913

[31] D. Teaney and L. Yan, Phys. Rev. C 83 (2011) 064904

[32] M. Luzum, Phys. Lett. B 696 (2011) 499

[33] S. Manly et al. (PHOBOS Collaboration), Nucl. Phys. A 774 (2006) 523

[34] K. Aamodt et al. (ALICE Collaboration), Phys. Rev. Lett. 107 (2011) 032301

[35] A. Adare et al. (PHENIX Collaboration), Phys. Rev. Lett. 107 (2011) 252301

[36] K. Aamodt et al. (ALICE Collaboration), Phys. Lett. B 708 (2012) 249

[37] G. Aad et al. (ATLAS Collaboration), Phys. Rev. C 86 (2012) 014907 
[38] CMS Collaboration, Measurement of higher-order harmonic flow in PbPb collisions at $\sqrt{s_{\mathrm{NN}}}=2.76 \mathrm{Te} V$, CMS PAS HIN-11-005

[39] CMS Collaboration, Azimuthal anisotropy harmonics in ultra-central $\mathrm{PbPb}$ collisions at $\sqrt{s_{\mathrm{NN}}}=2.76 \mathrm{Te} V$, CMS PAS HIN-12-011

[40] P. Huovinen et al., Phys. Lett. B 503 (2001) 58

[41] P. Kolb and U. Heinz, Quark-Gluon Plasma 3 (World Scientific, Singapore, 2004) 634-714

[42] D. Teaney, J. Lauret, and E. Shuryak, Nucl. Phys. A 698 (2002) 479

[43] P.K. Kovtun, D.T. Son, and A.O. Starinets, Phys. Rev. Lett. 94 (2005) 111601

[44] R. Glauber, Nucl. Phys. B 21 (1970) 135

[45] M.L. Miller et al., Ann. Rev. Nucl. Part. Sci. 57 (2007) 205

[46] S.S. Adler et al. (PHENIX Collaboration), Phys. Rev. Lett. 94 (2005) 232301

[47] K. Adcox et al. (PHENIX Collaboration), Phys. Rev. Lett. 88 (2001) 022301

[48] M. Gyulassy and L. McLerran, Nucl. Phys. A 750 (2005) 30

[49] I. Vitev, J. Phys. Conf. Ser. 50 (2006) 119

[50] A. Adare et al. (PHENIX Collaboration), Phys. Rev. Lett. 104 (2010) 132301

[51] D. d'Enterria and D. Peressounko, Eur. Phys. J. C 46 (2006) 451

[52] T. Matsui and H. Satz, Phys. Lett. B 178 (1986) 416

[53] M.C. Abreu et al. (NA50 Collaboration), Phys. Lett. B 521 (2001) 195

[54] S. Gavin and R. Vogt, Phys. Rev. Lett. 78 (1997) 1006

[55] C. Spieles et al., Phys. Rev. C 60 (1999) 054901

[56] A. Capella, A.B. Kaidalov, and D. Sousa, Phys. Rev. C 65 (2002) 054908

[57] P. Braun-Munzinger and J. Stachel, Phys. Lett. B 490 (2000) 196

[58] P. Braun-Munzinger and J. Stachel, Nucl. Phys. A 690 (2001) 119

[59] R.L. Thews, M. Schroedter, and J. Rafelski, Phys. Rev. C 63 (2001) 054905

[60] A. Andronic et al., Nucl. Phys. A 789 (2007) 334

[61] A. Adare et al. (PHENIX Collaboration), Phys. Rev. Lett. 98 (2007) 232301

[62] A. Andronic et al., Phys. Lett. B 659 (2008) 149

[63] K. Aamodt et al. (ALICE Collaboration), Phys. Lett. B 696 (2011) 328

[64] K. Aamodt et al. (ALICE Collaboration), Phys. Rev. Lett. 105 (2010) 252301

[65] K. Aamodt et al. (ALICE Collaboration), Phys. Rev. Lett. 105 (2010) 252302

[66] G. Aad et al. (ATLAS Collaboration), Phys. Lett. B 707 (2012) 330

[67] S. Chatrchyan et al. (CMS Collaboration), Phys. Rev. C 87 (2013) 014902

[68] K. Aamodt et al. (ALICE Collaboration), Phys. Lett. B 696 (2011) 30

[69] S. Chatrchyan et al. (CMS Collaboration), Eur. Phys. J. C 72 (2012) 1945

[70] B. Abelev et al. (ALICE Collaboration), Phys. Rev. Lett. 109 (2012) 072301

[71] R. Reed et al. (STAR Collaboration), J. Phys. G: Nucl. Part. Phys. 38 (2011) 124185

[72] S. Chatrchyan et al. (CMS Collaboration), JHEP 05 (2012) 063

[73] K. Nakamura et al. (Particle Data Group), J. Phys. G: Nucl. Part. Phys. 37 (2010) 075021

[74] J.A. Appel, Ann. Rev. Nucl. Part. Sci. 42 (1992) 367 
[75] R.K. Ellis, W.J. Stirling, and B.R. Webber, QCD and Collider Physics (Cambridge University Press, Cambridge, 1996)

[76] M. Mangano, Int. School of Physics E. Fermi, Course 137, arXiv:hep-ph/9711337

[77] R. Vogt, Phys. Rep. 310 (1999) 197

[78] N. Brambilla et al. (QWG), Heavy Quarkonium Physics arXiv:hep-ph/0412158

[79] C. Lourenco and H. Satz (Eds.), Proceedings on the International Conference on Hard and Electromagnetic Probes of High Energy Nuclear Collisions, Eur. Phys. J. C 43 (2005)

[80] R. Rapp, D. Blaschke, and P. Crochet, Prog. Part. Nucl. Phys. 65 (2010) 209

[81] M. Cacciari, M. Greco, and P. Nason, JHEP 9805 (1998) 007

[82] M. Cacciari, S. Frixione, and P. Nason, JHEP 0103 (2001) 006

[83] M. Cacciari et al., JHEP 10 (2012) 137

[84] B.A. Kniehl et al., Phys. Rev. Lett. 96 (2006) 012001

[85] B. Kniehl et al., Phys. Rev. Lett. 97 (2006) 252002

[86] A. Accardi et al., Hard probes in heavy ion collisions at the LHC: PDFs, shadowing and $p A$ collisions, arXiv:hep-ph/0308248

[87] F. Gelis, T. Lappi, and R. Venugopolan, Int. J. Mod. Phys. E 16 (2007) 2595

[88] S. Forte, Acta Phys. Polon. B 41 (2010) 2859

[89] M. Gyulassy and M. Plumer, Phys. Lett. B 243 (1990) 432

[90] A. Majumder and M. Van Leeuwen, Prog. Part. Nucl. Phys. 66 (2011) 41

[91] A. Dainese, C. Loizides, and G. Paic, Eur. Phys. J. C 38 (2005) 461

[92] C. Loizides, Eur. Phys. J. C 49 (2007) 339

[93] M. Gyulassy, P. Levai, and I. Vitev, Nucl. Phys. B 571 (2000) 197

[94] S. Wicks et al., Nucl. Phys. A 784 (2007) 426

[95] H. Zhang et al., Phys. Rev. Lett. 98 (2007) 212301

[96] R. Baier et al., Nucl. Phys. B 484 (1997) 265

[97] C.A. Salgado and U.A. Wiedemann, Phys. Rev. D 68 (2003) 014008

[98] Y.L. Dokshitzer and D.E. Kharzeev, Phys. Lett. B 519 (2001) 199

[99] M.G. Mustafa, Phys. Rev. C 72 (2005) 014905

[100] G.D. Moore and D. Teaney, Phys. Rev. C 71 (2005) 064904

[101] H. van Hees, V. Greco, and R. Rapp, Phys. Rev. C 73 (2005) 034913

[102] A. Adil and I. Vitev, Phys. Lett. B 649 (2007) 139

[103] R. Sharma, I. Vitev, and B. Zhang, Phys. Rev. C 80 (2009) 054902

[104] W.A. Horowitz, AIP Conf. Proc 1441 (2012) 889

[105] S.S. Adler et al. (PHENIX Collaboration), Phys. Rev. Lett. 91 (2003) 172301

[106] I. Arsene et al. (BRAHMS Collaboration), Phys. Rev. C 72 (2005) 014908

[107] B. Abelev et al. (STAR Collaboration), Phys. Rev. Lett. 97 (2006) 152301

[108] P. Sorensen and X. Dong, Phys. Rev. C 74 (2006) 024902

[109] G. Martinez-Garcia, S. Gadrat, and P. Crochet, Phys. Lett. B 663 (2008) 55 and Phys. Lett. B 666 (2008) 533

[110] J.J Aubert et al., Phys. Rev. Lett. 33 (1974) 1404 
[111] J.E. Augustin et al., Phys. Rev. Lett. 33 (1974) 1406

[112] S.W. Herb et al., Phys. Rev. Lett. 39 (1977) 252

[113] G. Goldhaber et al., Phys. Rev. Lett. 37 (1976) 265

[114] I. Peruzzi et al., Phys. Rev. Lett. 37 (1976) 569

[115] C. Bebek et al. (CLEO Collaboration), Phys. Rev. Lett. 46 (1981) 84

[116] L.J. Spencer et al. (CUSB Collaboration), Phys. Rev. Lett. 47 (1981) 771

[117] F.W. Büsser et al., Phys. Lett. B 53 (1974) 212

[118] C. Lourenco and H.K. Wöhri, Phys. Rep. 433 (2006) 127

[119] G.A. Alves et al., Phys. Rev. Lett. 77 (1977) 2388

[120] T. Sjostrand, S. Mrenna, and P. Skands, JHEP 0605 (2006) 026

[121] P. Braun-Munzinger et al., Eur. Phys. J. C 1 (1998) 123

[122] C. Lourenco et al. (NA38 Collaboration), Nucl. Phys. A 566 (1994) 77c

[123] A.L.S. Angelis et al. (HELIOS-3 Collaboration), Eur. Phys. J. C 13 (2000) 433

[124] M.C. Abreu et al. (NA38 and NA50 Collaborations), Eur. Phys. J. C 14 (2000) 443

[125] M.C. Abreu et al. (NA50 Collaboration), Phys. Lett. B 410 (1997) 327

[126] M. Keil et al., Nucl. Instrum. Methods A 549 (2005) 20

[127] R. Arnaldi et al. (NA60 Collaboration), Eur. Phys. J. C 59 (2009) 607

[128] R. Arnaldi et al. (NA60 Collaboration), Eur. Phys. J. C 61 (2009) 711

[129] B. Friman et al. (Eds.), Springer Lecture Notes in Physics 814 (2011)

[130] C.W. Fabjan and N. McCubbin, Phys. Rep. 403-404 (2004) 165

[131] C. Albajar et al. (UA1 Collaboration), Phys. Lett. B 186 (1987) 237

[132] C. Albajar et al. (UA1 Collaboration), Phys. Lett. B 256 (1991) 121 and Phys. Lett. B 262 (1991) 497

[133] P. Nason, S. Dawson, and R.K. Ellis, Nucl. Phys. B 303 (1988) 607

[134] P. Nason, S. Dawson, and R.K. Ellis, Nucl. Phys. B 327 (1989) 49 and Nucl. Phys. B 335 (1990) 260

[135] W. Beenakker et al., Phys. Rev. D 40 (1989) 54

[136] W. Beenakker et al., Nucl. Phys. B 351 (1991) 507

[137] C. Albajar et al. (UA1 Collaboration), Phys. Lett. B 244 (1990) 566

[138] O. Botner et al. (UA2 Collaboration), Phys. Lett. B 236 (1990) 488

[139] N. Ellis and A. Kernan, Phys. Rep. 195 (1990) 23

[140] F. Abe et al. (CDF Collaboration), Phys. Rev. Lett. 68 (1992) 3403

[141] F. Abe et al. (CDF Collaboration), Phys. Rev. Lett. 71 (1993) 500

[142] F. Abe et al. (CDF Collaboration), Phys. Rev. Lett. 71 (1993) 2396

[143] F. Abe et al. (CDF Collaboration), Phys. Rev. D 50 (1994) 4252

[144] S. Abachi et al. (D0 Collaboration), Phys. Rev. Lett. 74 (1995) 3548

[145] B. Abbott et al. (D0 Collaboration), Phys. Lett. B 487 (2000) 264

[146] B. Abbott et al. (D0 Collaboration), Phys. Rev. Lett. 84 (2000) 5478

[147] S. Abachi et al. (D0 Collaboration), Phys. Lett. B 370 (1996) 239

[148] M. Cacciari, arXiv:hep-ph/0407187 
[149] M.L. Mangano, AIP Conf. Proc. 753 (2005) 247

[150] D. Acosta et al. (CDF Collaboration), Phys. Rev. D 71 (2005) 032001

[151] D. Acosta et al. (CDF Collaboration), Phys. Rev. Lett. 91 (2003) 241804

[152] A.D. Frawley, T. Ullrich, and R. Vogt, Phys. Rep. 462 (2008) 128

[153] M. Harrison, T. Ludlam, and S. Ozaki (Eds.), The Relativistic Heavy Ion Collider Project: RHIC and its Detectors Nucl. Instrum. Methods A 499 (2003) 469-602

[154] http://www.phenix.bnl.gov

[155] A. Adare et al. (PHENIX Collaboration), Phys. Rev. C 84 (2011) 044905

[156] A. Adare et al. (PHENIX Collaboration), Phys. Rev. C 86 (2012) 024909

[157] K. Adcox et al. (PHENIX Collaboration), Phys. Rev. Lett. 88 (2002) 192303

[158] S.S. Adler et al. (PHENIX Collaboration), Phys. Rev. Lett. 94 (2005) 082301

[159] S.S. Adler et al. (PHENIX Collaboration), Phys. Rev. Lett. 96 (2006) 032001

[160] S.S. Adler et al. (PHENIX Collaboration), Phys. Rev. Lett. 96 (2006) 032301

[161] A. Adare et al. (PHENIX Collaboration), Phys. Rev. Lett. 97 (2006) 252002

[162] A. Adare et al. (PHENIX Collaboration), Phys. Rev. Lett. 98 (2007) 172301

[163] A. Adare et al. (PHENIX Collaboration), Phys. Rev. Lett. 103 (2009) 082002

[164] A. Adare et al. (PHENIX Collaboration), Phys. Lett. B 670 (2009) 313

[165] A. Adare et al. (PHENIX Collaboration), Phys. Rev. C 81 (2010) 034911

[166] A. Adare et al. (PHENIX Collaboration), Phys. Rev. D 76 (2007) 092002

[167] M. Harrison, T. Ludlam, and S. Ozaki (Eds.), The Relativistic Heavy Ion Collider Project: RHIC and its Detectors Nucl. Instrum. Methods A 499 (2003) 624-813

[168] http://www.star.bnl.gov and J. Dunlop, private communication

[169] J. Adams et al. (STAR Collaboration), Phys. Rev. Lett. 94 (2005) 062301

[170] L. Adamczyk et al. (STAR Collaboration), Phys. Rev. D 86 (2012) 072013

[171] B.I. Abelev et al. (STAR Collaboration), arXiv:0805.0364

[172] B.I. Abelev et al. (STAR Collaboration), Phys. Rev. D 79 (2009) 112006

[173] B.I. Abelev et al. (STAR Collaboration), Phys. Rev. Lett. 98 (2007) 192301 and Phys. Rev. Lett. 106 (2011) 159902

[174] H. Agakishiev et al. (STAR Collaboration), Phys. Rev. D 83 (2011) 052006

[175] M.M. Aggarwal et al. (STAR Collaboration), Phys. Rev. Lett. 105 (2010) 202301

[176] D. Lange, Nucl. Instrum. Methods A 462 (2001) 152

[177] C. Jaroschek, Master Thesis, Stony Brook University, 2001

[178] D. Kharzeev, Y.V. Kovchegov, and K. Tuchin, Phys. Rev. D 68 (2003) 094013

[179] J.W. Cronin et al., Phys. Rev. D 11 (1975) 3105

[180] P.B. Straub et al., Phys. Rev. Lett. 68 (1992) 452

[181] A. Adare et al. (PHENIX Collaboration), Phys. Rev. Lett. 109 (2012) 242301

[182] I. Arsene et al. (BRAHMS Collaboration), Phys. Rev. Lett. 91 (2003) 072305

[183] S.S. Adler et al. (PHENIX Collaboration), Phys. Rev. Lett. 91 (2003) 072303

[184] B.B. Back et al. (PHOBOS Collaboration), Phys. Rev. Lett. 91 (2003) 072302

[185] J. Adams et al. (STAR Collaboration), Phys. Rev. Lett. 91 (2003) 072304 
[186] S.S. Adler et al. (PHENIX Collaboration), Phys. Rev. C 74 (2006) 024904

[187] M. Basile et al., Nuovo Cimento Soc. Ital. Fis. 65A (1981) 421

[188] M. Mangano, P. Nason, and G. Ridolfi, Nucl. Phys. B 405 (1993) 507

[189] M. Djordjevic et al., Phys. Lett. B 632 (2006) 81

[190] N. Armesto et al., Phys. Lett. B 637 (2006) 362

[191] S.S. Adler et al. (PHENIX Collaboration), Phys. Rev. Lett. 91 (2003) 072301

[192] J. Adams et al. (STAR Collaboration), Phys. Rev. Lett. 91 (2003) 172301

[193] S.S. Adler et al. (PHENIX Collaboration), Phys. Rev. Lett. 96 (2006) 032302

[194] O. Linnyk, E. Bratkovskaya, and W. Cassing, Int. J. Mod. Phys. E 17 (2008) 1367

[195] P.B. Gossiaux and J. Aichelin, Phys. Rev. C 78 (2008) 014904

[196] P.B. Gossiaux, R. Bierkandt, and J. Aichelin, Phys. Rev. C 79 (2009) 044906

[197] P.B. Gossiaux and J. Aichelin, J. Phys. G: Nucl. Part. Phys. 36 (2009) 064028

[198] H. van Hees et al., Phys. Rev. Lett. 100 (2008) 192301

[199] P. Danielexicz and M. Gyulassy, Phys. Rev. D 31 (1985) 53

[200] R.A. Lacey et al., Phys. Rev. Lett. 98 (2007) 092301

[201] H.J. Drescher et al., Phys. Rev. C 76 (2007) 024905

[202] S. Gavin and M. Abdel-Aziz, Phys. Rev. Lett. 97 (2006) 162302

[203] A. Dumitru, E. Molnar, and Y. Nara, Phys. Rev. C 76 (2007) 024910

[204] P. Romatschke and U. Romatschke, Phys. Rev. Lett. 99 (2007) 172301

[205] M. Luzum and P. Romatschke, Phys. Rev. C 78 (2008) 034915 and Phys. Rev. C 79 (2009) 039903(E)

[206] S. Gubser, Phys. Rev. D 74 (2006) 126005

[207] C.P. Herzog et al., JHEP 07 (2006) 013

[208] W.A. Horowitz and M. Gyulassy, Phys. Lett. B 666 (2008) 320

[209] K. Aamodt et al. (ALICE Collaboration), J. Instrum. 3 (2008) S08002

[210] B. Abelev et al. (ALICE Collaboration), JHEP 01 (2012) 128

[211] B. Abelev et al. (ALICE Collaboration), JHEP 11 (2012) 065

[212] B. Abelev et al. (ALICE Collaboration), Phys. Rev. D 86 (2012) 112007

[213] B. Abelev et al. (ALICE Collaboration), Phys. Lett. B 717 (2012) 162

[214] K. Koch et al. (ALICE Collaboration), Nucl. Phys. A 855 (2011) 281

[215] K. Aamodt et al. (ALICE Collaboration), Phys. Lett. B 704 (2011) 442

[216] V. Khachatryan et al. (CMS Collaboration), Eur. Phys. J. C 71 (2011) 1575

[217] V. Khachatryan et al. (CMS Collaboration), Phys. Rev. D 83 (2011) 112004

[218] L.E. Gordon and W. Vogelsang, Phys. Rev. D 48 (1993) 3136

[219] L.E. Gordon and W. Vogelsang, Phys. Rev. D 50 (1994) 1901

[220] B. Abelev et al. (ALICE Collaboration), submitted for publication in Phys. Lett. B, arXiv:1208.1902

[221] B. Abelev et al. (ALICE Collaboration), Phys. Lett. B 708 (2012) 265

[222] B. Abelev et al. (ALICE Collaboration), Phys. Rev. Lett. 109 (2012) 112301 
[223] H. Appelshäuser et al. (ALICE C0llaboration), J. Phys. G: Nucl. Part. Phys. 38 (2011) 124014

[224] G. Aad et al. (ATLAS Collaboration), J. Instrum. 3 (2008) S08003

[225] S. Chatrchyan et al. (CMS Collaboration), J. Instrum. 3 (2008) S08004

[226] A. Augusto Alves Jr. et al. (LHCb Collaboration), J. Instrum. 3 (2008) S08005

[227] S. Chatrchyan et al. (CMS Collaboration), Phys. Rev. Lett. 106 (2011) 252001

[228] V. Khachatryan et al. (CMS Collaboration), Phys. Rev. Lett. 106 (2011) 112001

[229] S. Chatrchyan et al. (CMS Collaboration), Phys. Rev. D 84 (2011) 052008

[230] S. Frixione, P. Nason, and B.R. Webber, JHEP 08 (2003) 007

[231] R. Aaij et al. (LHCb Collaboration), JHEP 04 (2012) 093

[232] R. Aaij et al. (LHCb Collaboration), Phys. Lett. B 694 (2010) 209

[233] J.M. Campbell and K. Ellis, MCFM - Monte Carlo for FeMtobarn processes, http://mcfm.fnal.gov/

[234] G. Aad et al. (ATLAS Collaboration), Nucl. Phys. B 850 (2011) 387

[235] S. Chatrchyan et al. (CMS Collaboration), JHEP 2 (2012) 011

[236] R. Aaij et al. (LHCb Collaboration), Eur. Phys. J. C 71 (2011) 1645

[237] G. Aad et al. (ATLAS Collaboration), Phys. Lett. B 707 (2012) 438

[238] S. Frixione, P. Nason, and C. Oleari, JHEP 0711 (2007) 070

[239] S. Alioli et al.JHEP 1006 (2010) 043

[240] G. Corcella et al., JHEP 0101 (2001) 010

[241] V. Khachatryan et al. (CMS Collaboration), JHEP 03 (2011) 090

[242] R. Averbeck et al., arXiv:1107.3243

[243] B. Abelev et al. (ALICE Collaboration), JHEP 1207 (2012) 191

[244] B. Abelev et al. (ALICE Collaboration), JHEP 09 (2012) 112

[245] K.J. Eskola, H. Paukkunen, and C.A. Salgado, JHEP 0904 (2009) 065

[246] B. Abelev et al. (ALICE Collaboration), submitted for publication, arXiv:1208.2711

[247] S. Chatrchyan et al. (CMS Collaboration), JHEP 1205 (2012) 063

[248] Y. He, I. Vitev, and B.W. Zhang, Phys. Lett. B 713 (2012) 224

[249] W.A. Horowitz and M. Gyulassy, J. Phys. G: Nucl. Part. Phys. 38 (2011) 124114

[250] A. Buzzatti and M. Gyulassy, Phys. Rev. Lett. 108 (2012) 022301

[251] W.M. Alberico et al., Eur. Phys. J. C 71 (2011) 1666

[252] W.M. Alberico et al., J. Phys. G: Nucl. Part. Phys. 38 (2011) 124144

[253] P.B. Gossiaux et al., J. Phys. G: Nucl. Part. Phys. 37 (2010) 094019

[254] O. Fochler et al., J. Phys. G: Nucl. Part. Phys. 38 (2011) 124152

[255] N. Armesto et al., Phys. Rev. D 71 (2005) 054027

[256] C. Bianchin et al. (ALICE Collaboration), Acta Phys. Polon. Proc. Suppl. B 5 (2012) 335

[257] Y. Pachmayer et al. (ALICE Collaboration), J. Phys. G: Nucl. Part. Phys. 38 (2011) 124186 